\title{
Analysis of CHP Potential at Federal Sites
}

\section{February 2002}

S. W. Hadley

K. L. Kline

S. E. Livengood

J. W. Van Dyke 


\section{DOCUMENT AVAILABILITY}

Reports produced after January 1, 1996, are generally available free via the U.S. Department of Energy (DOE) Information Bridge:

Web site: http://www.osti.gov/bridge

Reports produced before January 1, 1996, may be purchased by members of the public from the following source:

National Technical Information Service

5285 Port Royal Road

Springfield, VA 22161

Telephone: 703-605-6000 (1-800-553-6847)

TDD: $703-487-4639$

Fax: 703-605-6900

E-mail: info@ntis.fedworld.gov

Web site: http://www.ntis.gov/support/ordernowabout.htm

Reports are available to DOE employees, DOE contractors, Energy Technology Data Exchange (ETDE) representatives, and International Nuclear Information System (INIS) representatives from the following source:

Office of Scientific and Technical Information

P.O. Box 62

Oak Ridge, TN 37831

Telephone: 865-576-8401

Fax: 865-576-5728

E-mail: reports@adonis.osti.gov

Web site: http://www.osti.gov/contact.html

This report was prepared as an account of work sponsored by an agency of the United States Government. Neither the United States government nor any agency thereof, nor any of their employees, makes any warranty, express or implied, or assumes any legal liability or responsibility for the accuracy, completeness, or usefulness of any information, apparatus, product, or process disclosed, or represents that its use would not infringe privately owned rights. Reference herein to any specific commercial product, process, or service by trade name, trademark, manufacturer, or otherwise, does not necessarily constitute or imply its endorsement, recommendation, or favoring by the United States Government or any agency thereof. The views and opinions of authors expressed herein do not necessarily state or reflect those of the United States Government or any agency thereof. 
S. W. Hadley

K. L. Kline

S. E. Livengood

J. W. Van Dyke

February 2002

Prepared for the

U.S. Department of Energy

Office of Energy Efficiency and Renewable Energy

Federal Energy Management Program

\author{
OAK RIDGE NATIONAL LABORATORY \\ Oak Ridge, Tennessee 37831 \\ managed by \\ UT-BATTELLE, LLC \\ for the \\ U.S. DEPARTMENT OF ENERGY \\ under contract no. DE-AC05-00OR22725
}


This page intentionally left blank. 


\section{CONTENTS}

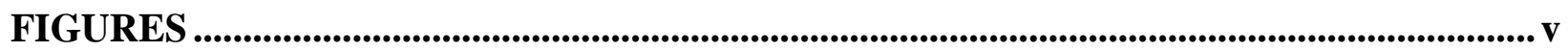

TABLES .......................................................................................................................................................... vii

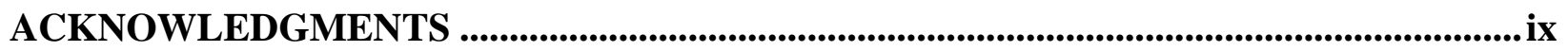

FOREWORD ........................................................................................................................................

EXECUTIVE SUMMARY ...................................................................................................ii

1 Introduction .............................................................................................................................................. 1

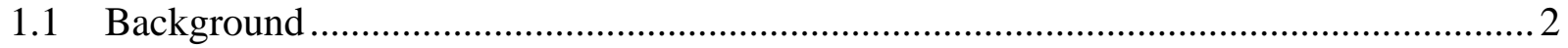

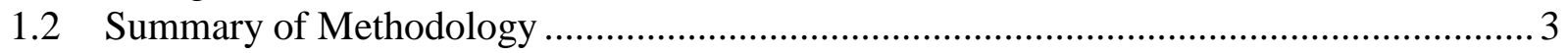

2 Data Sources for Analysis ....................................................................................................................5

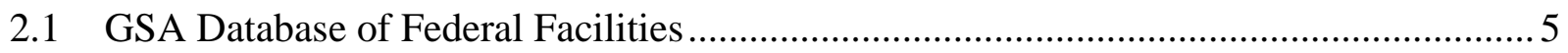

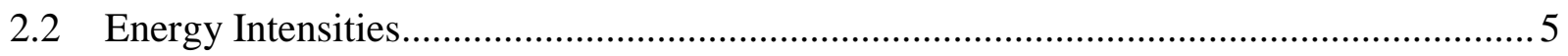

2.3 Retail Gas and Electric Prices ................................................................................. 8

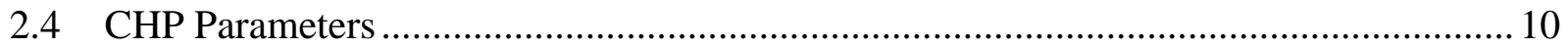

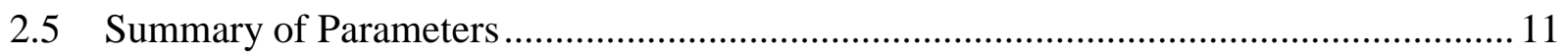

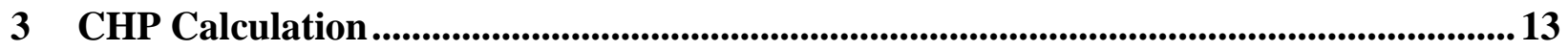

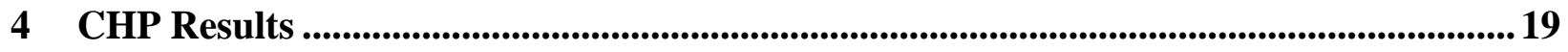

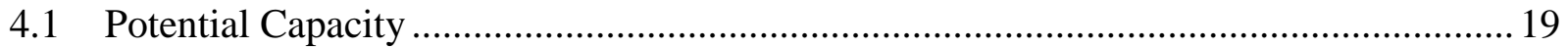

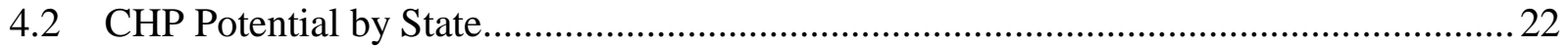

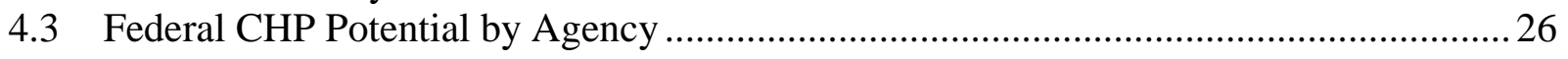

5 Sensitivity Analysis ............................................................................................................29

6 Conclusions ............................................................................................................................................... 33

6.1 Data Limitations and Further Studies ……………….................................................... 33

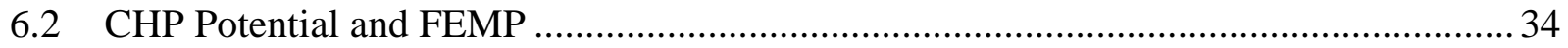

6.3 How to Determine Whether a Facility Has CHP Potential ............................................. 36

References ................................................................................................................................................................ 37

Appendix A: GSA Federal Building Data Base Categories Defined ........................................A-1

Appendix B: Methods Used to Perform Statistical Analysis on CBECS 95 .......................... B-1

Appendix C: Utility Interconnection Requirements, Exit Fees, Stand-By Fees....................C-1

Appendix D: Emissions Permitting and Siting ................................................................................D-1 
This page intentionally left blank. 


\section{FIGURES}

S-1: CHP systems recover usable heat and avoid transmission and distribution losses to

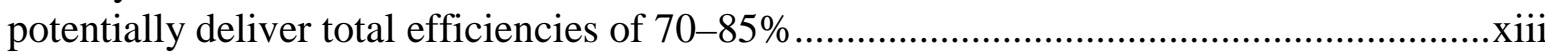

S-2: Potential CHP capacity for major federal agencies, MW ............................................... Xv

S-3: Potential CHP capacity in federal sites under different technologies and performance

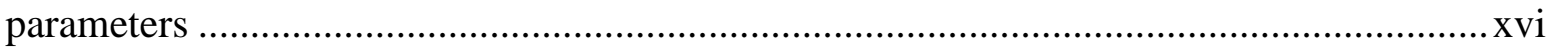

S-4: Distribution of potential CHP capacity in federal sites under base case, MW ..................xvii

1: National CHP Roadmap_objectives for 2000-2010 ...................................................... 1

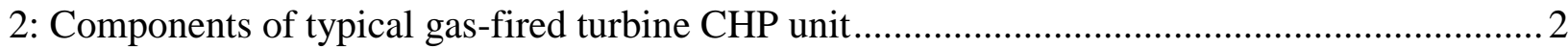

3: A comparison of providing equivalent electric and heat using CHP or conventional

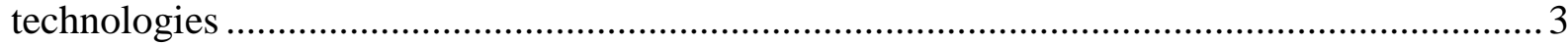

4: Energy flows of a combined cooling, heat, and power system .......................................... 10

5: Load curve template for office building and CHP unit used during occupation .................... 14

6: Load curve template for hospital with two load-following CHP units ................................ 15

7: Comparison of electricity and steam needs for Iowa Methodist Medical Center ................... 15

8: Effect of including cost of money in payback calculation ................................................ 17

9: Percent of federal sites with CHP potential by building category and corresponding

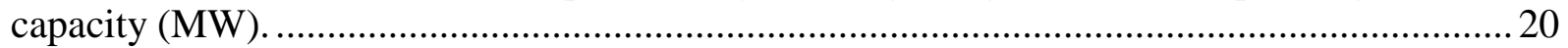

10: CHP potential capacity by building type for top 20 states, MW ......................................22

11: Federal CHP potential capacity under base case, MW .................................................25

12: "Spark spread" difference in electric and gas prices in $\$ / \mathrm{MBtu}$.........................................25

13: Potential CHP capacity for major federal agencies (\% of $1588 \mathrm{MW}$ total) .........................2

14: CHP potential capacity (MW) under varying cost and efficiency assumptions ....................30

15: Potential CHP capacity in federal sites under different technologies and performance

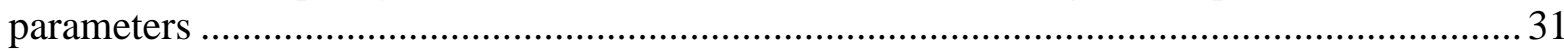

16: CHP capacity in base case at different ranges of payback period..................................... 32

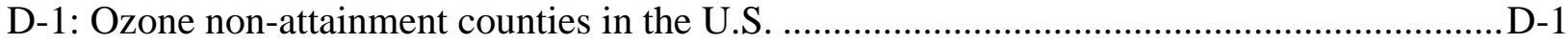


This page intentionally left blank. 


\section{TABLES}

S-1: National CHP potential at federal facilities larger than $25,000 \mathrm{ft}^{2}$ using base case assumptions xiv

1: Building types from CBECS with associated GSA types .............................................. 6

2: Electric site energy intensities $\left(\mathrm{kBtu} / \mathrm{ft}^{2}\right)$ for buildings greater than $100,000 \mathrm{ft}^{2} \ldots \ldots \ldots \ldots \ldots \ldots \ldots .6$

3: Gas site energy intensities $\left(\mathrm{kBtu} / \mathrm{ft}^{2}\right)$ for buildings greater than $100,000 \mathrm{ft}^{2} \ldots \ldots \ldots \ldots \ldots \ldots \ldots \ldots . . \ldots$

4: Electric site energy intensities $\left(\mathrm{kBtu} / \mathrm{ft}^{2}\right)$ for buildings between 25,000 and 100,000 $\mathrm{ft}^{2} \ldots \ldots \ldots .7$

5: Gas site energy intensities $\left(\mathrm{kBtu} / \mathrm{ft}^{2}\right)$ for buildings between 25,000 and 100,000 $\mathrm{ft}^{2} \ldots \ldots \ldots \ldots \ldots .7$

6: Percentage of building type using natural gas and central heating (district hot water

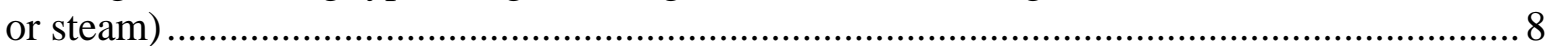

7: Electric and gas commercial and industrial prices for 1999 and 2000 ................................. 9

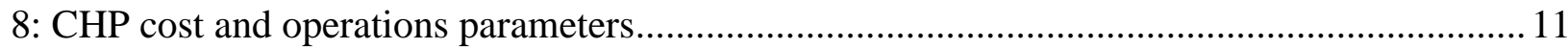

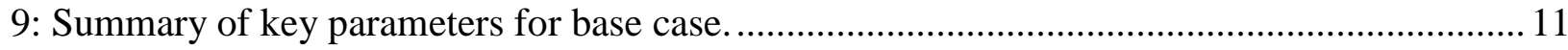

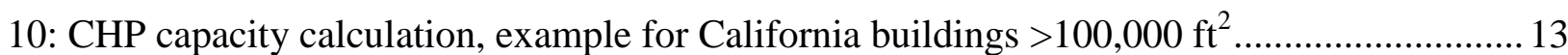

11: National CHP potential by building category at federal facilities using base case assumptions

12: National CHP costs, savings, and payback, by building category, under base case assumptions 21

13: Site and source energy savings from CHP, TBtu/year................................................. 22

14: State CHP potential capacity by building type under base case, MW ...............................23

15: Potential CHP capacity by federal agency and building category, MW ............................ 26

16: Potential CHP capacity by state for leading agencies .................................................. 28

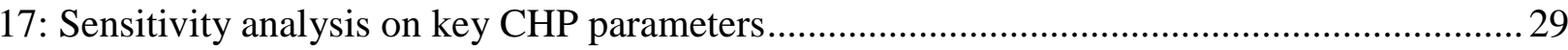

D-1. Example of technologies with potential to meet Texas emission regulations ................... D-2 
This page intentionally left blank. 


\section{ACKNOWLEDGMENTS}

The authors wish to thank everyone who supported the review and publication of this document, including: Elizabeth Shearer, Director, U.S. Department of Energy (DOE) Federal Energy Management Program (FEMP); Tatiana Strajnic, Alison Thomas, and Shawn Herrera at DOE-FEMP headquarters; Rich Combes, Lisa Hollingsworth, and Arun Jhaveri at DOE regional offices; Chris Tremper and Tim Rooney of McNeil Technologies for assistance with updated data sources; Patrick Hughes, Steve Fischer, and the CHP core team at ORNL for technical reviews and support; and Linda Stansberry and Missy Sherrod for administrative and editorial support. 
This page intentionally left blank. 


\section{FOREWORD}

This document was prepared at the request of the U.S. Department of Energy's (DOE's) Federal Energy Management Program (FEMP) under its Technical Guidance and Assistance and Project Financing Programs. The purpose was to provide an estimate of the national potential for combined heat and power (also known as CHP; cogeneration; or cooling, heating, and power) applications at federal facilities and the associated costs and benefits including energy and emission savings. The report provides a broad overview for the U.S. Department of Energy (DOE) and other agencies on when and where CHP systems are most likely to serve the government's best interest.

FEMP's mission is to reduce the cost to and environmental impact of the federal government by advancing energy efficiency and water conservation, promoting the use of renewable energy, and improving utility management decisions at federal sites. FEMP programs are driven by its customers: federal agency sites. FEMP monitors energy efficiency and renewable energy technology developments and mounts "technology-specific" programs to make technologies that are in strong demand by agencies more accessible. FEMP's role is often one of helping the federal government "lead by example" through the use of advanced energy efficiency/renewable energy (EERE) technologies in its own buildings and facilities.

CHP was highlighted in the Bush Administration's National Energy Policy Report as a commercially available technology offering extraordinary benefits in terms of energy efficiencies and emission reductions. FEMP's criteria for emphasizing a technology are that it must be commercially available; be proven but underutilized; have a strong constituency and momentum; offer large energy savings and other benefits of interest to federal sites and FEMP mission; be in demand; and carry sufficient federal market potential. As discussed in the report, CHP meets all of these criteria. Executive Order 13123 directs federal facilities to use CHP when life-cycle costs indicate energy reduction goals will be met. FEMP can assist facilities to conduct this analysis.

The model developed for this report estimates the magnitude of CHP that could be implemented under various performance and economic assumptions associated with different applications. This model may be useful for other energy technologies. It can be adapted to estimate the market potential in federal buildings for any energy system based on the cost and performance parameters that a user desires to assess. The model already incorporates a standard set of parameters based on available data for federal buildings including total building space, building type, energy use intensity, fuel costs, and the performance of many prime movers commonly used in CHP applications. These and other variables can be adjusted to meet user needs or updated in the future as new data become available. 
This page intentionally left blank. 


\section{EXECUTIVE SUMMARY}

Combined heat and power (also known as CHP; cogeneration; or cooling, heating, and power) can be used to provide thermal energy for buildings or processes while at the same time generating a portion of electricity needs. A CHP system recovers the heat from electricity generation for productive uses such as heating, cooling, dehumidification, or other processes. This heat is normally wasted by conventional power plants. And because a CHP system generates electricity near the point of use, CHP also avoids transmission and distribution losses from distant central stations. For these reasons, properly designed CHP systems can be much more efficient than the average U.S. fossil fuel power plant, as shown in Fig. S-1.

Fig. S-1. CHP systems recover usable heat and avoid transmission and distribution losses to potentially deliver total efficiencies of $70-85 \%$.

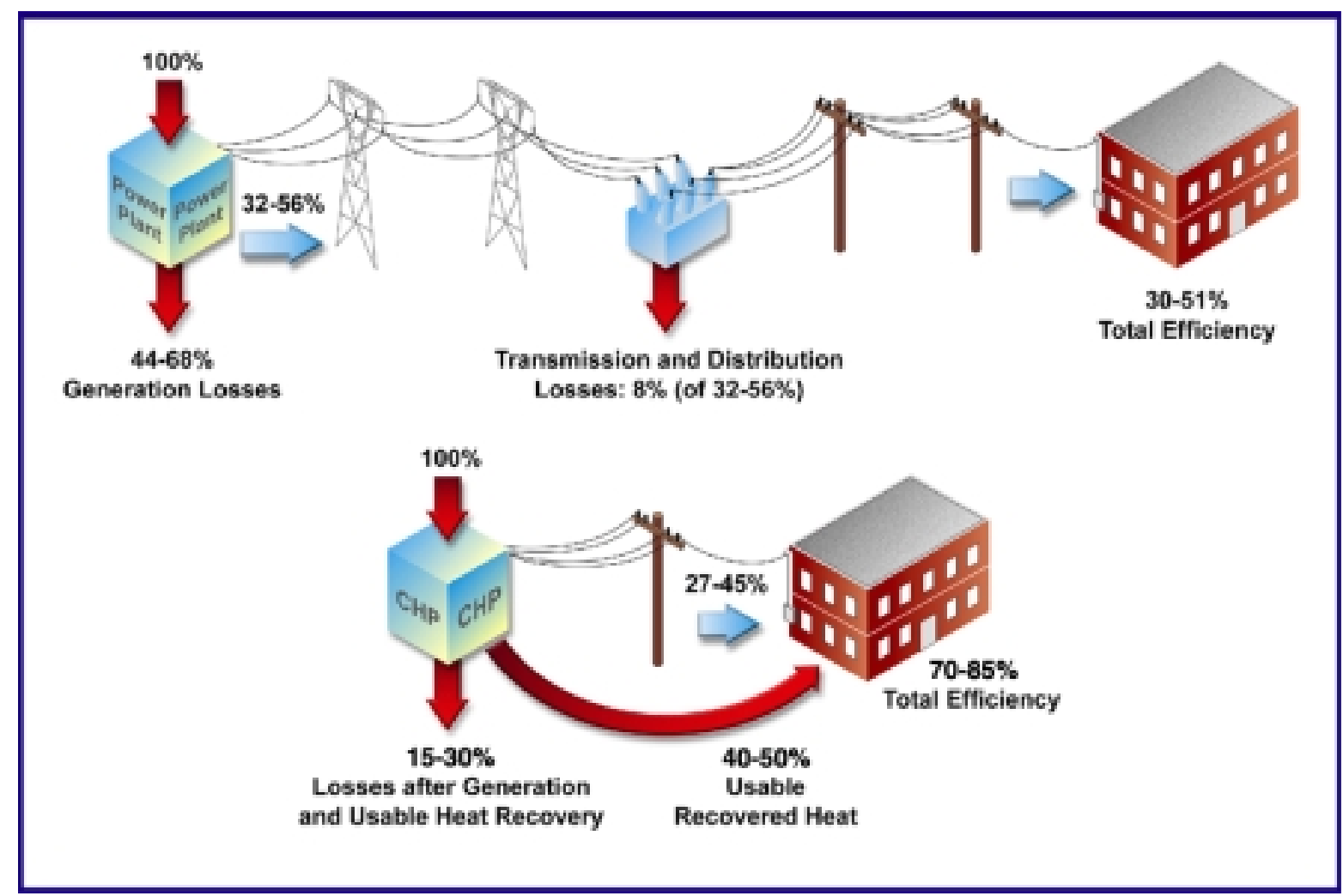

There has been a recent upsurge in interest in fuel-efficient distributed energy resources (DER) such as $\mathrm{CHP}$ among project developers, federal facility managers, and policy makers because these systems have the potential to significantly reduce key power sector constraints. They offer an opportunity to meet increased energy needs, reduce transmission congestion, cut emissions, increase power quality and reliability, and increase a facility's overall energy security. In sufficient numbers, interconnected CHP systems can offer increased power security for the grid as well (Casten and Casten 2001). CHP was highlighted in the Bush Administration's National Energy Policy Report as being commercially available and offering extraordinary benefits in terms of energy efficiencies and emission reductions. CHP in 
buildings facilitates a transition to cleaner fuels and technologies of the future (such as hydrogen and fuel cells) that would rely upon the same infrastructure as CHP.

Many questions arise regarding CHP in federal facilities: How much capacity is potentially available nationwide? Is it significant? Where and in which agencies is it concentrated? What are the economics involved? What difference does technology make? What types of buildings are the best candidates for CHP? To help answer these questions, staff at Oak Ridge National Laboratory (ORNL) created a model that calculates the energy use and costs in different types of federal buildings across the country. This model allows the user to select various parameters regarding the CHP technology, energy prices, and energy use for various building types. It then calculates the financial payback of CHP to determine the amount that could be implemented economically. The base case included only those buildings with simple paybacks of less than ten years.

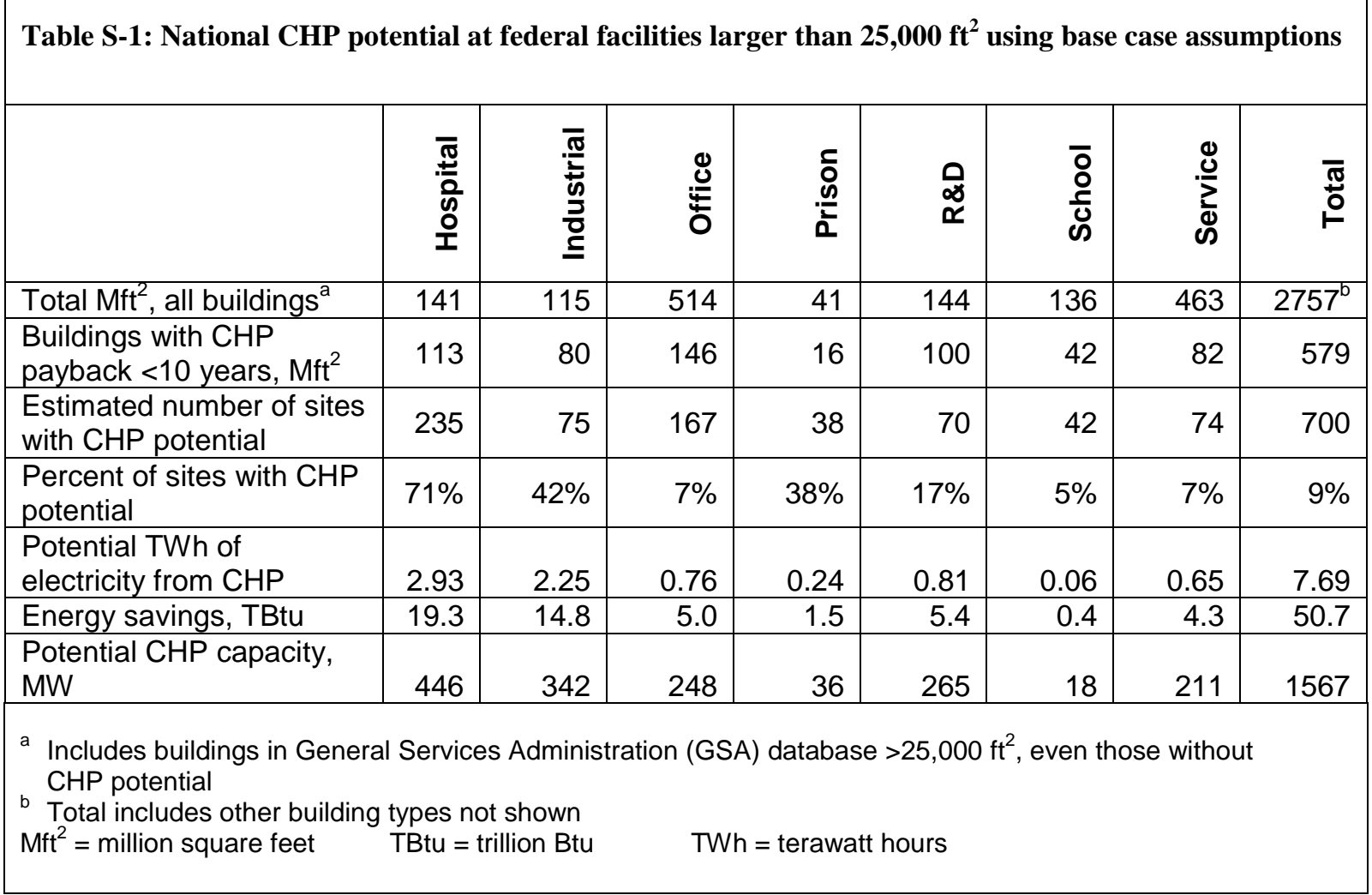

Total potential CHP capacity was estimated to be 1500-1600 MW under the assumptions and parameters used for this analysis, using gas reciprocating engine or gas combustion turbine technologies in federal facilities across the country. Electricity potentially produced with this capacity represents approximately $13 \%$ of all electric use in the federal sector (FEMP 2002). The federal building types with CHP potential were primarily hospitals, industrial and R\&D facilities. Table S-1 summarizes analysis results including the amount of capacity and savings for each building category studied. The assessment considered 7 building types for 28 different federal agencies. Figure S-2 shows the calculated amount of CHP capacity for the 9 major agencies; the others each had capacities of less than $10 \mathrm{MW}$. Not surprisingly, the military branches had highest overall CHP potential in most building categories. Concentrations of potential CHP capacity at the various federal agencies examined were as follows: the VA-hospitals; NASA and DOE-R\&D and industrial; General Services Administration (GSA) and Postal Service—offices; and the Justice Department—prisons. 
Fig. S-2: Potential CHP capacity for major federal agencies (MW).

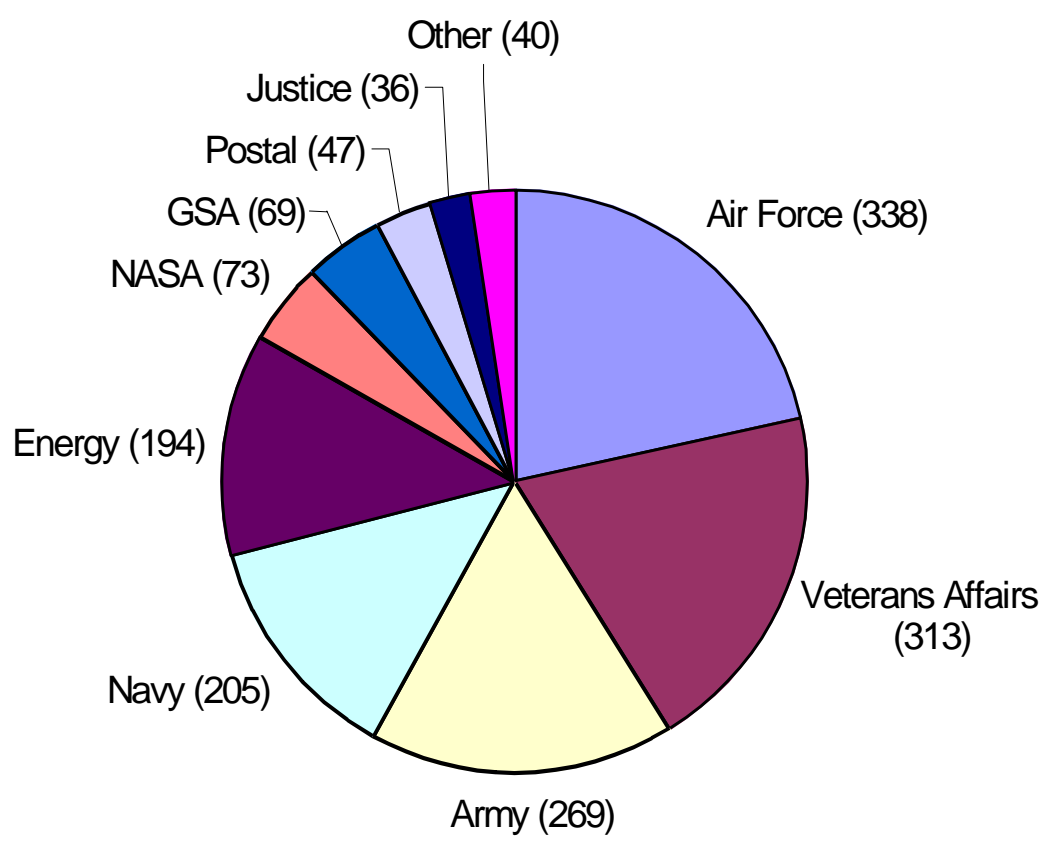

Sensitivity analysis of base case variables created widely varying estimates of potential capacity. Changing the performance and cost assumptions for CHP technology gave a range from 390 (doubling installed cost) to $2800 \mathrm{MW}$ using typical commercial power and gas rates instead of industrial rates. Using turbines with today's costs and efficiencies instead of the base case gas reciprocating engines gave similar results $(1670 \mathrm{MW})$, although future turbine efficiencies are expected to improve such that capacity with a less-than-10-year simple payback increases to $2400 \mathrm{MW}$, as shown in Fig. S-3. Fuel cells were assessed in the model but do not appear to be economical under present cost and performance parameters.

The authors acknowledge that the assessment methodology developed and utilized here is limited by the databases available to support it. For example, GSA's federal facility database does not always reflect recent changes in building ownership and use. It contains building-level data but no information on whether these buildings are served by district energy systems. District energy systems are a key indicator of CHP potential because they already have the infrastructure in place to supply thermal energy to multiple buildings. Where these systems exist, the significance of building types, which this study is based on, becomes secondary.

\section{Do you have CHP potential?}

Ideal sites will fit the following profile, but sites meeting only a few of these characteristics may also have a cost-effective CHP opportunity:

$\checkmark$ high electric prices (more than 5 cents/kWh);

$\checkmark$ average electric load greater than $1 \mathrm{MW}$;

$\checkmark$ ratio of average electric load to peak load $>0.7$

$\checkmark$ a central or district heating and/or cooling system in place (or a need for process heat)

$\checkmark$ "spark spread" (difference in price per million site Btu between gas and electricity) $>\$ 12$

$\checkmark$ high annual operating hours (>6000)

$\checkmark$ thermal demand closely matches electric load 
Fig. S-3: Potential CHP capacity in federal sites under different technologies and performance parameters.

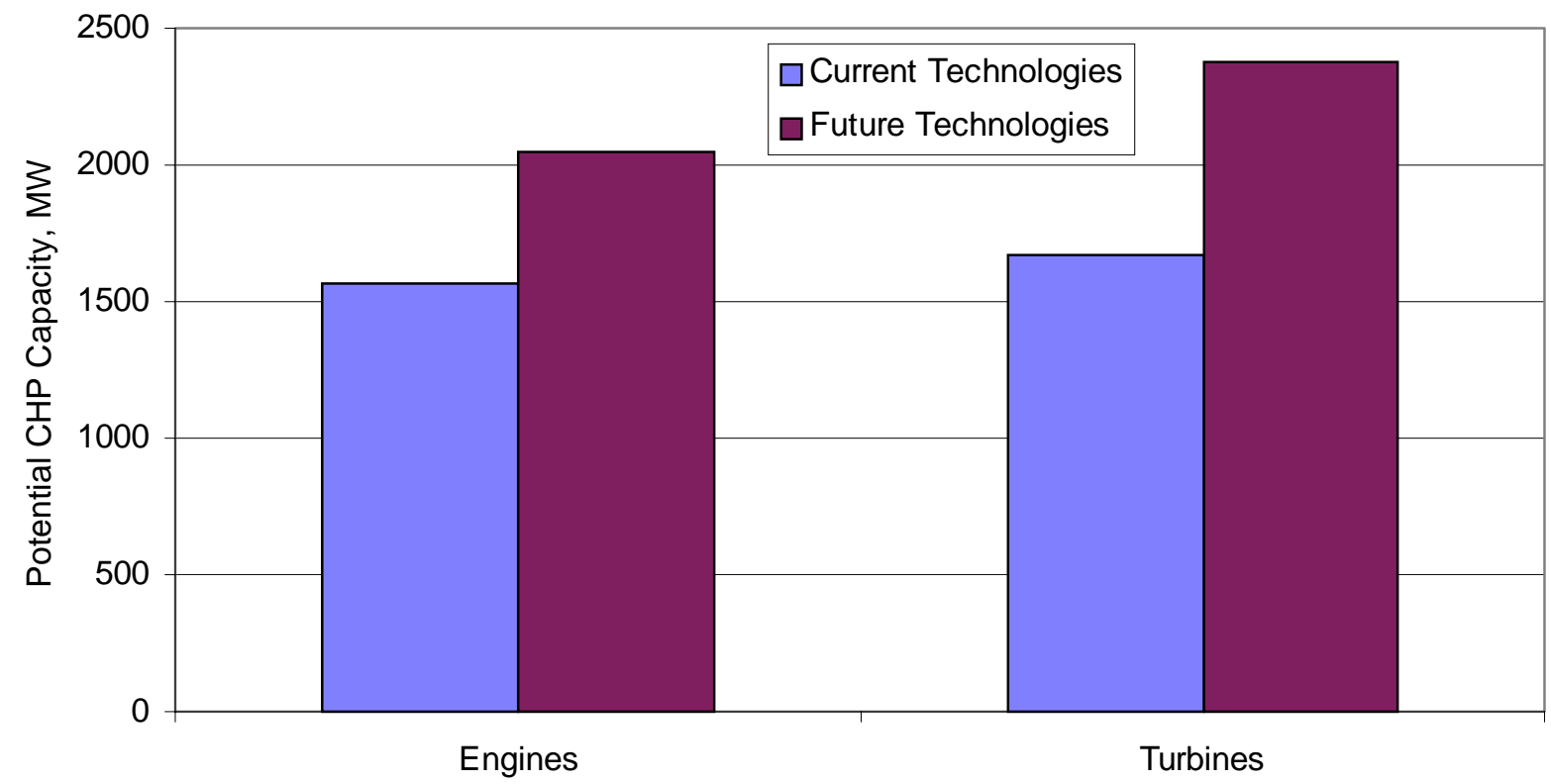

This study cannot attempt to identify which specific sites have CHP potential. Site-specific information is needed to identify the actual best candidates for federal CHP. This analysis used state and regional averages for many of the key parameters defining the amount of energy needed and price paid. The economics and feasibility of CHP are very site-specific because the condition of existing equipment, energy use at a facility, prices paid for electricity and gas, and local regulations related to emissions, interconnection, and siting can vary widely by site. However, this study does indicate the expected amounts of CHP and the most likely states, agencies, and building types for applications. And the model used for analysis of potential can easily generate results adjusted according to changing assumptions about energy prices and other variables. As energy prices increase and CHP system costs decrease, the amount of cost-effective CHP potential will rise. The actual numbers could be significantly higher or lower depending on the specific characteristics of any given site.

Our assessment reveals significant potential for CHP in the federal sector. The $1.5 \mathrm{GW}$ of estimated potential in the base-case scenario has an average simple payback of 6 years and could save the federal government $\$ 170 \mathrm{M}$ per year in energy costs. Given the large amount of potential for CHP at federal sites, why haven't more facilities installed this technology? Preliminary discussions with federal facility managers suggest the following primary reasons:

- historically low tariffs for electricity;

- high initial cost of CHP systems;

- limited budgets for capital improvement (agencies rarely have sufficient appropriations for even much smaller energy conservation investments);

- complexity of CHP systems due in part to the need for custom engineering and design of different components for each site;

- a lack of time and capability for facility managers to evaluate potential applications and benefits to their site;

- obstacles related to local regulations and policies for interconnection, standby/backup charges, siting, and emissions; and

- a lack of trusted sources of information about the costs, operation, and performance of CHP systems. 
The Federal Energy Management Program (FEMP) is collaborating to address many of these obstacles by offering unbiased information and technical and project financing assistance to any federal agency interested in developing a CHP project. FEMP CHP services, resources permitting, include:

- CHP quick technical screening for interested federal sites;

- site survey and feasibility verification;

- partnership building between federal sites and project developers that bring financing if needed;

- baseline data collection;

- link appropriate federal sites with industry teams developing "packaged" CHP systems;

- design and technical assistance to projects selected under FEMP calls for projects;

- support for addressing policy and regulatory constraints - siting and permitting, grid interconnection requirements, exit fees, standby/backup charges;

- verify designs, component matching, and system sizing to thermal and power profiles; and

- technical/price proposal evaluation

Under present assumptions, the regions with the greatest CHP potential are the Southwest (CA to TX), Northeastern metropolitan areas (NY to DC), and the Southeast (FL, GA, AL). Figure S-4 maps the potential capacity for each state. FEMP recognizes the potential for CHP to reduce the costs of government, increase energy security, and improve air quality, and is actively working to make advanced CHP technologies more easily accessible to federal agencies.

Fig. S-4: Distribution of potential CHP capacity in federal sites under base case, MW.

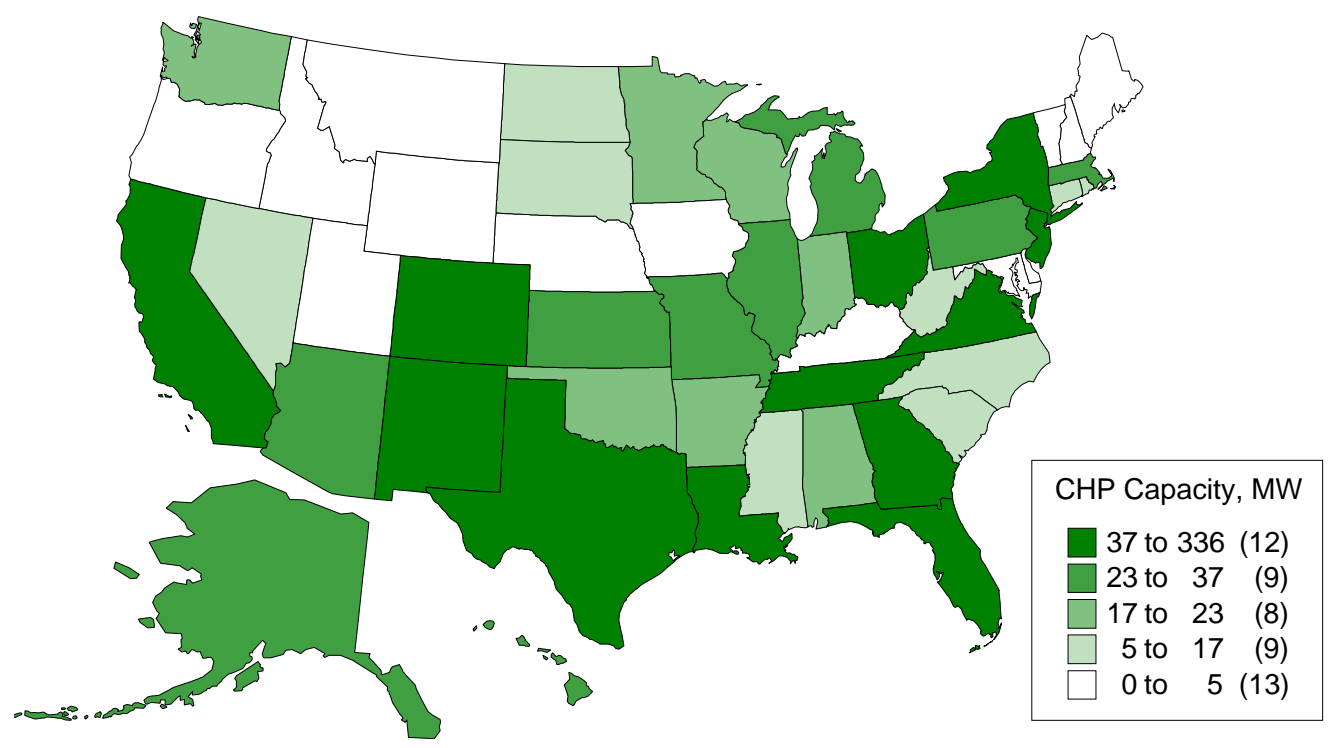


This page intentionally left blank. 


\section{Introduction}

CHP systems play an essential role in our nation's present energy supply and future plans. The United States has over 50 gigawatts (GW) of installed CHP capacity producing about $7 \%$ of the nation's electricity (USCHPA 2001). The Department of Energy (DOE) and the Environmental Protection Agency (EPA) have partnered with the private sector in an effort to double CHP capacity by 2010 because of the environmental and economic benefits offered (Fig. 1). The National Energy Policy Report focuses on the importance of CHP to help meet critical goals related to emissions reductions, energy security, reliability, and new energy production in a cost effective manner (NEPDG 2001). Federal agencies have a mandate to lead by example in meeting national energy and environmental goals, and an Executive Order specifies that agencies "shall use combined cooling, heat and power systems when life-cycle cost-effective" (FEMP 1999).

It is not surprising that there has been a recent upsurge in interest in $\mathrm{CHP}$ in federal facilities across the country. There is ample rationale to look carefully at the potential for CHP applications in the federal sector.

Fig. 1. National CHP roadmap-objectives for 2000-2010.

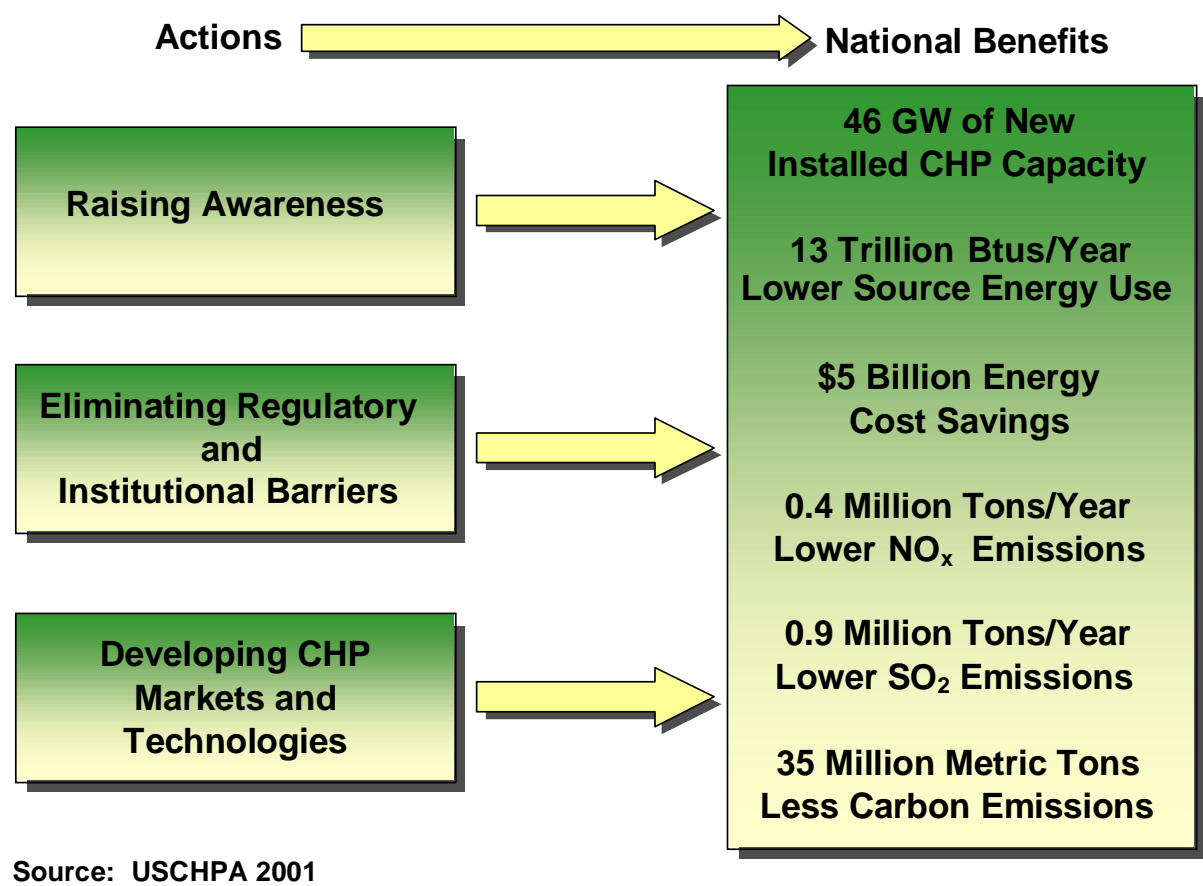

Source: USCHPA 2001 


\subsection{Background}

What is CHP? Combined cooling, heat, and power, also known as cogeneration or building cooling, heat and power (BCHP) is a system that efficiently generates electricity (or shaft power) and uses the heat generated in that process to produce steam, hot water, and/or hot air for other purposes. The most common building applications use a prime mover (turbine or engine) coupled with a generator to produce electricity and capture the waste heat for process steam and space heating and, when coupled to a chiller, to assist with space cooling or refrigeration.

CHP is based on system integration. A well-designed CHP plant integrates proven technologies for power generation (such as gas turbines or reciprocating engines) and thermal load management (chillers, dehumidifiers, boilers, other HVAC or process heat equipment) to maximize overall efficiency. Usually this involves sizing a system carefully to meet site-specific needs, taking into consideration existing equipment, fuel costs, electric and thermal load duration curves and other factors. CHP systems can be designed to make a site totally independent from the grid or, more commonly, to maximize savings and provide increased reliability for a strategic portion of the load at a site.

Reciprocating engine and generator sets have been supplying dependable power for over 50 years, so the technology is well known. Steam turbines that produce electricity from existing boiler capacity are also a thoroughly proven and highly reliable technology. Combustion gas turbines (over $500 \mathrm{~kW}$ in size), while more recent, have successfully penetrated the market over the past 20 years based on proven reliability, reduced emissions and competitive operation and maintenance costs. Smaller gas turbines and fuel cells are being demonstrated at many federal sites. Their present costs per installed $\mathrm{kW}$ of capacity are often higher than other options and their performance records are still being established.

The heat recovery systems are also well known and can be based on heat transfers from combustion exhaust, engine jackets, or other elements to either air or fluids. If exhaust heat can be transferred directly to an auxiliary unit (such as an absorption chiller or desiccant dehumidifier) it is called a "direct fired" application. More common are applications using steam or hot water. These systems normally use a heat recovery steam generator (HRSG) (see Fig. 2). There is substantial information available from manufacturers' and DOE websites regarding sizes, specifications, costs and performance of this equipment (such as that summarized later in Table 10 of this report).

Fig. 2. Components of typical gas-fired turbine CHP unit.

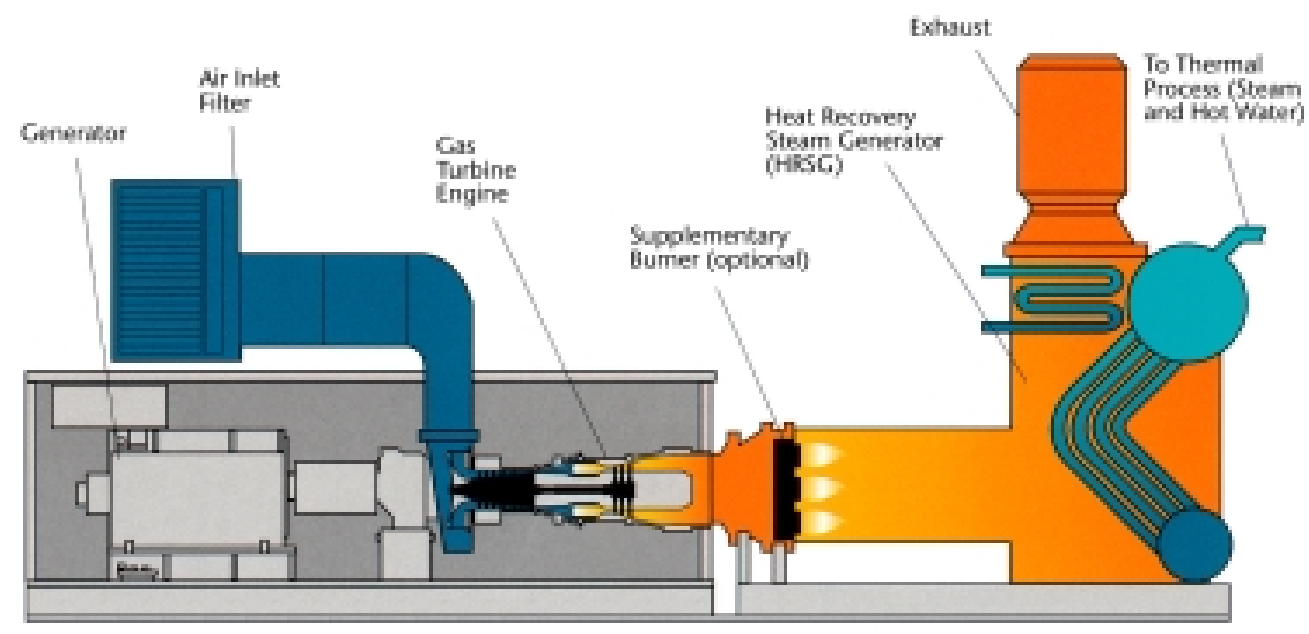

Graphic (adapted) courtesy of Solar Turbines Corp. 
In a typical federal installation such as those modeled for the market assessment, CHP is assumed to provide thermal energy for heating and cooling a building while at the same time generating a portion of its electricity needs. Because it uses the waste heat from electricity generation for the other functions, it is much more efficient at generating power than distant central stations. Figure 3 compares equivalent electric and heat using CHP or conventional technologies.

While other applications (process steam for industry, laboratories, laundry, hot water, dehumidification) and more complicated systems (thermal storage, multiple units of variable sizes and types, multiple thermal applications) are possible and often result as site specific conditions are analyzed, these alternatives were not considered in the assessment.

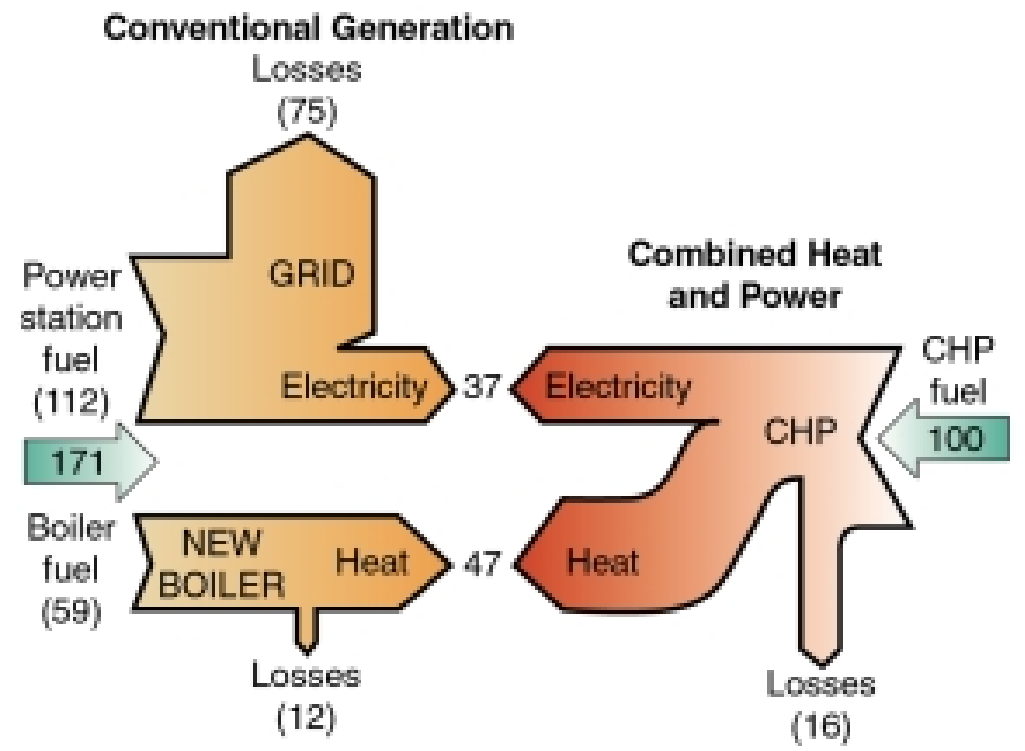

Fig. 3. A comparison of providing equivalent electric and heat using CHP or conventional technologies. Source (adapted): http://www.eren.doe.gov/der/combined_heat_power.html

Many questions arise regarding CHP in federal facilities. How much capacity is potentially available nationwide, and is it significant? Where and in which agencies is it concentrated? What are the economics involved? What difference does technology make? What types of buildings are most favorable? To help answer these questions, staff at Oak Ridge National Laboratory (ORNL) created a model that calculates the energy use and costs in different types of federal buildings across the country. The user can select various parameters regarding CHP and energy use for the various building types. Then the financial benefits can be calculated to determine the capacity of CHP that could be implemented within a defined payback period.

\subsection{Summary of Methodology}

We started with the General Services Administration (GSA 2001) database of all federal facilities, grouping the buildings by type and state. Separately, we calculated energy intensities from the Energy Information Administration (EIA) Commercial Building Energy Consumption Survey (CBECS) (EIA 
1998) database for each building type, building size, and region of the country. Analyzing data from these two sources allowed us to estimate the gas and electricity use for each federal building across the country.

The EIA releases retail energy prices for each state in several publications (EIA 2001). Resource Data International compiles the electric sector data into a convenient database known as PowerDat (RDI 2001). Selecting either the commercial or industrial rates for a given year, we calculated the cost of energy for each building. Using the size and energy use of the building, along with input parameters for the CHP technology, we calculated the amount and cost of energy use with CHP. Comparing the difference in cost with and without the CHP revealed the annual savings. Based on the type of CHP, the capital and installation cost can also be calculated. Dividing the annual savings by the cost gives the simple payback, while a more detailed payback that uses inflation and the cost of capital was also revealed. Those buildings with no cost savings or with simple paybacks longer than ten years were rejected, and the remaining potential CHP projects were summed for each state. 


\section{Data Sources for Analysis}

\subsection{GSA Database of Federal Facilities}

There are two datasets of federal facilities available. The Federal Energy Management Program (FEMP) maintains a data set called FEMPTracks. This dataset lists sites with number of buildings and square feet by type of building. It also has site-specific data on energy use and prices, but only for some sites. The data set includes 5800 records (with multiple buildings per record) and has a structure and content based on a dataset maintained by the GSA.

This GSA dataset provides a more comprehensive list of sites, containing 37,000 records, but does not include energy data. For this study, we used the GSA dataset. While the FEMPTracks data include more information on energy, the amount and quality of information was uncertain. It appears that both data sets have limitations in terms of timely updates to reflect new construction and decommissioning or change in facility use.

The GSA dataset was reorganized to sum all of the square feet and number of buildings by building type for each site. This reduced the number of records to 21,000 separate sites across the country. Appendix A contains GSA definitions for the 11 building categories.

\section{Building categories in GSA database}

- Hospital - Housing - Industrial

- Office - Prison - Other institution

- R\&D - School - All other

- Storage - Services

Besides square feet and number of buildings for each type, the dataset included site-specific information such as location and owner. The location information was used to assign the energy intensity and power prices from other datasets.

\subsection{Energy Intensities}

Every five years the EIA conducts a survey of commercial buildings across the U.S. The most recent surveyed 5766 buildings in 1995 (EIA 1998). This dataset contains a large amount of information on the buildings, including energy use by type of fuel, equipment installed, main end-uses for energy, and envelope characteristics. It includes the square feet for each building, as well as a weighting factor to indicate what percentage of all buildings each record represents.

The CBECS dataset includes 29 different building types as opposed to the 11 in the GSA dataset. Those that most closely matched the GSA types were used as shown in Table 1. CBECS does not include industrial facilities. Given the high energy intensity of industrial facilities, for this analysis, we assumed that they and GSA R\&D facilities were similar to the "laboratory" category of CBECS. The "Other" category in CBECS may not necessarily match the "Other" and "Other Institution" categories in the GSA dataset. There are also obvious differences between "Federal Prisons" in the GSA data set (which tend to be large, "24/7" facilities) versus the "Public Order and Safety" category in CBECS, which includes many smaller buildings. Because of the way the data were analyzed, this difference will result in a more conservative estimate of the amount of CHP potential. Also, "housing" in GSA refers primarily to military housing, versus "lodging" (hotel/motel/dorm) in CBECS. 
Table 1: Building types from CBECS with associated GSA types

\begin{tabular}{ll}
\hline GSA Category & CBECS Category \\
\hline Hospital & Health care (inpatient) \\
Housing & Lodging (hotel/motel/dorm) \\
Industrial & Laboratory \\
Office & Office/professional \\
Prison & Public order and safety \\
Other institution & Other \\
RD\&D & Laboratory \\
School & Education \\
Services & Service (excludes food) \\
Storage & Warehouse (non- \\
All other & refrigerated) \\
\hline
\end{tabular}

Electric and gas intensities were calculated for each of the building types for each region of the country and for two different sizes $\left(25,000\right.$ to $100,000 \mathrm{ft}^{2}$ and greater than $\left.100,000 \mathrm{ft}^{2}\right)$. Despite the large initial survey size, segregating the data by region, type, and size left some data categories with no samples. In those cases, we used the value from the other building sizes but the same type and region. If other sizes for that region were unavailable, we used the national average of the energy intensity for that building type. Tables 2 through 5 show the gas and electric energy intensities used (thousands of Btu per square foot).

Table 2: Electric site energy intensities $\left(\mathrm{kBtu} / \mathrm{ft}^{2}\right)$ for buildings greater than $100,000 \mathrm{ft}^{2}$

\begin{tabular}{|c|c|c|c|c|c|c|c|c|c|c|}
\hline Electric & 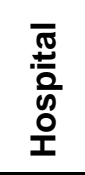 & $\begin{array}{l}\text { 옴 } \\
\text { 올 } \\
\text { 운 }\end{array}$ & $\begin{array}{l}\bar{\sigma} \\
\underline{\underline{2}} \\
\underline{0} \\
\underline{\partial} \\
\underline{0}\end{array}$ & $\underbrace{\stackrel{0}{0}}_{0}$ & 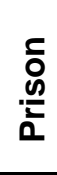 & $\begin{array}{l}\stackrel{亠}{ \pm} \\
\stackrel{ \pm}{0}\end{array}$ & $\underset{\underset{\sim}{\not}}{\stackrel{0}{\not}}$ & 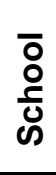 & 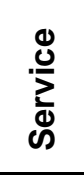 & $\begin{array}{l}\text { ठ } \\
\text { के } \\
\text { के }\end{array}$ \\
\hline$\overline{\text { All }}$ & 107 & 49 & 117 & 78 & 45 & 59 & 117 & 28 & 82 & 21 \\
\hline Northeast & 71 & 254 & 84 & 48 & 47 & 59 & 84 & 20 & 28 & 22 \\
\hline Mid-Atlantic & 85 & 53 & 94 & 56 & 37 & 113 & 94 & 23 & 49 & 24 \\
\hline E-N-Central & 118 & 40 & 99 & 70 & 52 & 71 & 99 & 22 & 21 & 14 \\
\hline W-N-Central & 96 & 57 & 110 & 73 & 12 & 40 & 110 & 29 & 251 & 27 \\
\hline South Atlantic & 125 & 51 & 179 & 82 & 58 & 56 & 179 & 38 & 65 & 15 \\
\hline E-S-Central & 137 & 17 & 104 & 85 & 45 & 81 & 104 & 52 & 8 & 34 \\
\hline W-S-Central & 112 & 70 & 117 & 84 & 78 & 59 & 117 & 47 & 85 & 20 \\
\hline Mountain & 92 & 41 & 117 & 102 & 45 & 59 & 117 & 41 & 62 & 16 \\
\hline Pacific & 101 & 48 & 111 & 104 & 71 & 19 & 111 & 32 & 59 & 26 \\
\hline
\end{tabular}

Source: EIA 1998, A Look at Commercial Buildings in 1995: Characteristics, Energy Consumption, and Energy Expenditures 
Table 3: Gas site energy intensities $\left(\mathrm{kBtu} / \mathrm{ft}^{2}\right)$ for buildings greater than $100,000 \mathrm{ft}^{2}$

\begin{tabular}{|c|c|c|c|c|c|c|c|c|c|c|}
\hline Gas & 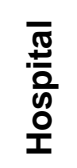 & $\begin{array}{l}\text { 옴 } \\
\text { 옴 } \\
\text { 운 }\end{array}$ & 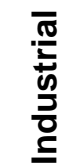 & $\frac{8}{0}$ & 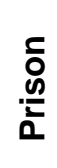 & $\begin{array}{l}\frac{\grave{d}}{c} \\
\bar{c}\end{array}$ & $\stackrel{Q}{\infty}$ & $\begin{array}{l}\overline{8} \\
\frac{0}{\mathcal{U}} \\
\dot{\omega}\end{array}$ & $\frac{\text { J }}{\stackrel{0}{2}}$ & 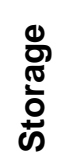 \\
\hline$\overline{\text { All }}$ & 162 & 67 & 134 & 30 & 36 & 60 & 134 & 36 & 75 & 18 \\
\hline Northeast & 176 & 63 & 41 & 32 & 96 & 60 & 41 & 34 & 98 & 27 \\
\hline Mid-Atlantic & 159 & 69 & 104 & 22 & 28 & 35 & 104 & 29 & 13 & 18 \\
\hline E-N-Central & 237 & 104 & 168 & 38 & 59 & 91 & 168 & 48 & 76 & 24 \\
\hline W-N-Central & 174 & 84 & 132 & 66 & 52 & 109 & 132 & 47 & 104 & 29 \\
\hline South Atlantic & 125 & 37 & 179 & 28 & 4 & 5 & 179 & 30 & 36 & 12 \\
\hline E-S-Central & 135 & 61 & 122 & 35 & 36 & 18 & 122 & 26 & 22 & 18 \\
\hline W-S-Central & 107 & 90 & 134 & 20 & 34 & 60 & 134 & 20 & 161 & 7 \\
\hline Mountain & 182 & 36 & 134 & 32 & 36 & 60 & 134 & 50 & 169 & 18 \\
\hline Pacific & 158 & 60 & 35 & 19 & 6 & 60 & 35 & 30 & 25 & 14 \\
\hline
\end{tabular}

Source: EIA 1998, A Look at Commercial Buildings in 1995: Characteristics, Energy Consumption, and Energy

Expenditures

Table 4: Electric site energy intensities $\left(\mathrm{kBtu} / \mathrm{ft}^{2}\right)$ for buildings between 25,000 and $100,000 \mathrm{ft}^{2}$

\begin{tabular}{|c|c|c|c|c|c|c|c|c|c|c|}
\hline Electric & $\begin{array}{l}\bar{\sigma} \\
\frac{\pi}{0} \\
\frac{0}{0} \\
\text { 우 }\end{array}$ & 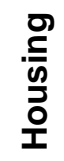 & 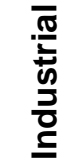 & $\underbrace{\stackrel{0}{0}}_{0}$ & 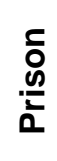 & $\frac{\grave{d}}{\vdots}$ & $\begin{array}{l}\not{\not} \\
\underset{\sim}{x}\end{array}$ & $\begin{array}{l}\overline{0} \\
\stackrel{\circ}{0} \\
\text { 옹 }\end{array}$ & $\frac{\mathbb{J}}{\frac{1}{2}}$ & 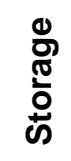 \\
\hline All & 93 & 50 & 138 & 63 & 62 & 37 & 138 & 27 & 41 & 20 \\
\hline Northeast & 87 & 50 & 138 & 32 & 24 & 37 & 138 & 18 & 29 & 19 \\
\hline Mid-Atlantic & 241 & 46 & 77 & 62 & 50 & 25 & 77 & 26 & 14 & 34 \\
\hline E-N-Central & 54 & 33 & 134 & 51 & 41 & 24 & 134 & 20 & 39 & 20 \\
\hline W-N-Central & 61 & 36 & 79 & 70 & 53 & 48 & 79 & 24 & 50 & 62 \\
\hline South Atlantic & 54 & 53 & 298 & 58 & 61 & 45 & 298 & 28 & 65 & 17 \\
\hline E-S-Central & 93 & 62 & 90 & 68 & 62 & 37 & 90 & 36 & 44 & 12 \\
\hline W-S-Central & 114 & 91 & 138 & 59 & 42 & 37 & 138 & 32 & 39 & 19 \\
\hline Mountain & 109 & 51 & 138 & 79 & 62 & 37 & 138 & 30 & 62 & 11 \\
\hline Pacific & 136 & 46 & 130 & 72 & 18 & 37 & 130 & 32 & 7 & 16 \\
\hline
\end{tabular}

Source: EIA 1998, A Look at Commercial Buildings in 1995: Characteristics, Energy Consumption, and Energy

Expenditures

Table 5: Gas site energy intensities $\left(\mathrm{kBtu} / \mathrm{ft}^{2}\right)$ for buildings between 25,000 and $100,000 \mathrm{ft}^{2}$

\begin{tabular}{|c|c|c|c|c|c|c|c|c|c|c|}
\hline Gas & $\begin{array}{l}\bar{\pi} \\
\frac{\pi}{0} \\
\text { on } \\
\text { 우 }\end{array}$ & $\begin{array}{l}\text { 옴 } \\
\text { 옴 } \\
\text { 운 }\end{array}$ & 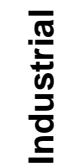 & 兽 & 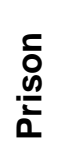 & $\frac{\grave{\Phi}}{\Phi}$ & $\begin{array}{l}\text { Q } \\
\underset{\sim}{\boldsymbol{x}}\end{array}$ & $\begin{array}{l}\overline{0} \\
\text { O্ } \\
\bar{d}\end{array}$ & 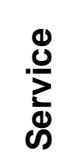 & $\begin{array}{l}\text { श् } \\
\text { के } \\
\text { ஸे }\end{array}$ \\
\hline All & 162 & 66 & 81 & 40 & 34 & 58 & 81 & 46 & 86 & 25 \\
\hline Northeast & 151 & 63 & 81 & 30 & 14 & 58 & 81 & 46 & 98 & 33 \\
\hline Mid-Atlantic & 368 & 60 & 9 & 39 & 1 & 38 & 9 & 37 & 39 & 24 \\
\hline E-N-Central & 162 & 80 & 80 & 65 & 64 & 19 & 80 & 72 & 51 & 44 \\
\hline W-N-Central & 22 & 80 & 3 & 46 & 97 & 159 & 3 & 39 & 87 & 39 \\
\hline South Atlantic & 99 & 60 & 224 & 12 & 27 & 31 & 224 & 21 & 36 & 21 \\
\hline E-S-Central & 162 & 63 & 21 & 17 & 34 & 58 & 21 & 42 & 92 & 20 \\
\hline W-S-Central & 64 & 34 & 81 & 24 & 13 & 58 & 81 & 30 & 161 & 9 \\
\hline Mountain & 669 & 51 & 81 & 58 & 34 & 58 & 81 & 58 & 169 & 19 \\
\hline Pacific & 36 & 76 & 128 & 27 & 52 & 58 & 128 & 55 & 86 & 6 \\
\hline
\end{tabular}

Source: EIA 1998, A Look at Commercial Buildings in 1995: Characteristics, Energy Consumption, and Energy

Expenditures 
A second set of factors from the CBECS data set was also important. Not all commercial buildings have gas service and the configuration of HVAC equipment and distribution systems necessary to easily adopt a CHP system. We calculated what percentage of each building type used natural gas and had central heating (either district hot water or steam) (Table 6). We separately calculated the percentage with central cooling or forced air systems, but decided that the most likely candidates would have a central heating system that could use the exhaust heat from a CHP unit. (Appendix B describes the calculations in more detail.) We then assumed that those percentages also applied to the federal buildings under analysis. This allowed us to estimate the percentage of each building category that was expected to be compatible with CHP. As with the energy intensities, the values for laboratories were used for industrial buildings.

The housing, storage, and "other" building types in CBECS did not necessarily reflect the categories in the GSA database, nor were they expected to offer significant CHP potential at this time, so they were excluded from this study. The study methodology also excludes from assessment a percent of floor space in each building category that is not likely to have infrastructure compatible with CHP. Note that in all categories except hospitals, a relatively small percentage of buildings under $100,000 \mathrm{ft}^{2}$ are expected to have infrastructure that facilitates CHP. While the lack of infrastructure does not necessarily preclude a cost-effective CHP project, it certainly reduces the probability. And since the costs to retrofit for thermal applications are so highly site-specific and variable, we decided it would be more realistic to limit CHP analysis to the percent of building stock for each category listed in Table 6 .

Table 6: Percentage of building type using natural gas and central heating (district hot water or steam)— assumed "CHP compatible" for this study (\%)

\begin{tabular}{|c|c|c|c|c|c|c|c|c|c|c|}
\hline & $\begin{array}{l}\bar{\varpi} \\
\overline{\frac{ \pm}{0}} \\
\text { 。 } \\
\text { 오 }\end{array}$ & $\begin{array}{l}\text { 옴 } \\
\text { 옴 } \\
\text { 옴 }\end{array}$ & 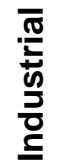 & $\frac{8}{0}$ & 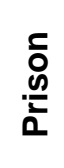 & 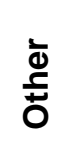 & $\underset{\sim}{\infty}$ & 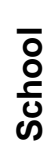 & 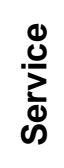 & $\begin{array}{l}\text { வ } \\
\frac{\pi}{0} \\
\text { ळ }\end{array}$ \\
\hline $\begin{array}{l}>100,000 \mathrm{ft}^{2} \\
<100,000 \mathrm{ft}^{2}\end{array}$ & $\begin{array}{l}88 \\
66 \\
\end{array}$ & & $\begin{array}{c}78 \\
7 \\
\end{array}$ & $\begin{array}{l}48 \\
11 \\
\end{array}$ & $\begin{array}{l}56 \\
16 \\
\end{array}$ & & $\begin{array}{c}78 \\
7\end{array}$ & $\begin{array}{l}71 \\
30 \\
\end{array}$ & $\begin{array}{c}24 \\
3\end{array}$ & \\
\hline
\end{tabular}

Source: EIA 1998, A Look at Commercial Buildings in 1995: Characteristics, Energy Consumption, and Energy Expenditures.

\subsection{Retail Gas and Electric Prices}

The EIA releases the retail prices for natural gas and electricity through several publications. We used the Natural Gas Monthly data set (EIA 2001) that contains monthly residential, commercial, and industrial natural gas prices for each state for recent years (Table 7). In addition to EIA data, we used the PowerDat dataset from Resource Data International (RDI 2001) to find the commercial and industrial electric prices for each state for 1999 and 2000. Our base case analysis assumed that federal facilities' prices were closest to the industrial rates, but for a sensitivity test we also used the commercial rates. We also included the capability to use 1999 or 2000 prices, because gas prices were unusually high in the latter part of the 2000. Actual rates at a given federal facility are often negotiated on a site-specific basis and can vary widely from commercial or industrial tariffs. 
Table 7: Electric and gas commercial and industrial prices for 1999 and 2000

\begin{tabular}{|c|c|c|c|c|c|c|c|c|}
\hline & \multicolumn{2}{|c|}{$\begin{array}{c}1999 \text { Gas Price } \\
\text { \$/MBtu }\end{array}$} & \multicolumn{2}{|c|}{$\begin{array}{c}2000 \text { Gas Price } \\
\$ / \text { Mbtu }\end{array}$} & \multicolumn{2}{|c|}{$\begin{array}{c}1999 \text { Electric Price } \\
\text { \$/MWh }\end{array}$} & \multicolumn{2}{|c|}{$\begin{array}{c}2000 \text { Electric Price } \\
\$ / \mathrm{MWh}\end{array}$} \\
\hline State & Comm. & Industrial & Comm. & Industrial & Comm. & Industrial & Comm. & Industrial \\
\hline AK & 2.19 & 1.25 & 2.06 & 1.57 & 81.25 & 66.78 & 81.35 & 70.34 \\
\hline $\mathbf{A L}$ & 6.73 & 3.43 & 7.79 & 4.41 & 65.64 & 39.89 & 66.17 & 40.92 \\
\hline AR & 5.40 & 3.44 & 5.40 & 3.87 & 56.55 & 43.51 & 57.60 & 44.44 \\
\hline $\mathbf{A Z}$ & 6.17 & 3.43 & 6.72 & 4.43 & 74.00 & 55.39 & 73.54 & 54.90 \\
\hline CA & 6.18 & 3.42 & 7.56 & 5.76 & 93.73 & 60.46 & 91.67 & 53.91 \\
\hline $\mathrm{CO}$ & 4.55 & 2.83 & 5.24 & 3.33 & 53.96 & 42.04 & 54.94 & 43.06 \\
\hline CT & 6.49 & 4.16 & 6.59 & 5.74 & 96.88 & 74.29 & 92.13 & 73.75 \\
\hline DC & 7.38 & 5.73 & 8.96 & 7.23 & 74.74 & 45.90 & 78.28 & 48.53 \\
\hline DE & 7.00 & 4.15 & 6.94 & 5.20 & 71.47 & 43.67 & 58.91 & 37.65 \\
\hline $\mathbf{F L}$ & 6.51 & 3.64 & 7.83 & 5.21 & 61.64 & 46.56 & 61.30 & 47.92 \\
\hline GA & 5.09 & 3.39 & 6.19 & 4.37 & 64.89 & 41.99 & 63.92 & 42.32 \\
\hline HI & 14.32 & 8.21 & 17.29 & 10.18 & 124.32 & 93.50 & 142.28 & 110.82 \\
\hline IA & 4.82 & 4.01 & 6.73 & 5.22 & 66.33 & 38.55 & 68.60 & 39.05 \\
\hline ID & 4.78 & 3.30 & 5.69 & 3.91 & 41.49 & 27.11 & 41.77 & 30.30 \\
\hline IL & 5.36 & 4.12 & 7.14 & 5.77 & 75.54 & 50.79 & 73.68 & 44.66 \\
\hline IN & 5.20 & 4.27 & 5.99 & 4.55 & 61.52 & 41.36 & 59.84 & 39.98 \\
\hline KS & 5.06 & 3.03 & 5.02 & 4.01 & 59.28 & 44.52 & 59.78 & 44.51 \\
\hline KY & 5.14 & 3.31 & 6.78 & 5.00 & 50.19 & 28.88 & 48.81 & 30.04 \\
\hline LA & 5.73 & 2.51 & 7.02 & 4.09 & 67.44 & 42.22 & 71.14 & 46.35 \\
\hline MA & 7.60 & 5.19 & 9.47 & 7.00 & 86.68 & 72.26 & 90.71 & 78.29 \\
\hline MD & 7.07 & 5.73 & 7.98 & 7.23 & 68.35 & 41.98 & 68.94 & 41.62 \\
\hline ME & 6.65 & 4.92 & 6.78 & 5.24 & 105.22 & 64.84 & 91.43 & 48.64 \\
\hline MI & 4.94 & 3.82 & 4.91 & 4.30 & 78.87 & 52.15 & 79.41 & 52.43 \\
\hline MN & 4.44 & 2.97 & 6.29 & 4.46 & 63.55 & 45.70 & 64.20 & 45.71 \\
\hline MO & 5.47 & 4.23 & 6.93 & 5.82 & 59.30 & 45.44 & 60.05 & 48.29 \\
\hline MS & 4.88 & 3.24 & 6.07 & 4.31 & 59.28 & 37.01 & 62.45 & 39.31 \\
\hline MT & 5.15 & 3.65 & 5.23 & 4.91 & 64.98 & 52.66 & 58.66 & 31.95 \\
\hline NC & 6.22 & 3.73 & 7.51 & 5.53 & 60.15 & 44.81 & 60.71 & 45.35 \\
\hline ND & 4.52 & 2.81 & 5.96 & 4.96 & 58.21 & 45.78 & 58.53 & 45.50 \\
\hline NE & 4.13 & 3.39 & 5.51 & 4.67 & 53.01 & 32.96 & 54.40 & 33.58 \\
\hline NH & 6.86 & 4.53 & 7.36 & 6.69 & 115.32 & 93.58 & 114.91 & 94.22 \\
\hline NJ & 3.98 & 3.10 & 5.31 & 4.18 & 98.00 & 77.70 & 87.05 & 67.76 \\
\hline NM & 3.83 & 2.41 & 4.68 & 3.88 & 76.26 & 43.12 & 69.20 & 47.19 \\
\hline NV & 6.05 & 4.78 & 5.56 & 5.35 & 67.43 & 48.08 & 67.18 & 49.69 \\
\hline NY & 5.10 & 3.68 & 5.24 & 5.10 & 115.63 & 47.32 & 129.50 & 48.30 \\
\hline $\mathrm{OH}$ & 5.63 & 4.00 & 7.03 & 6.03 & 75.68 & 42.33 & 74.49 & 44.17 \\
\hline OK & 5.16 & 3.50 & 6.40 & 5.15 & 53.16 & 35.06 & 58.62 & 39.54 \\
\hline OR & 5.66 & 4.02 & 6.42 & 4.54 & 50.21 & 33.85 & 51.21 & 35.89 \\
\hline PA & 7.32 & 3.97 & 7.38 & 4.82 & 66.96 & 42.76 & 63.85 & 40.95 \\
\hline $\mathbf{R I}$ & 8.19 & 4.53 & 8.32 & 5.45 & 82.76 & 66.56 & 94.20 & 82.83 \\
\hline SC & 6.54 & 3.39 & 7.92 & 4.88 & 61.11 & 36.67 & 60.29 & 36.27 \\
\hline SD & 4.56 & 3.41 & 5.93 & 4.35 & 67.67 & 45.96 & 66.92 & 46.40 \\
\hline TN & 5.73 & 3.76 & 6.73 & 5.39 & 62.86 & 43.76 & 62.70 & 44.54 \\
\hline $\mathrm{TX}$ & 4.44 & 2.49 & 5.48 & 3.99 & 64.74 & 40.61 & 66.72 & 43.96 \\
\hline UT & 4.11 & 2.97 & 4.89 & 3.69 & 51.68 & 33.02 & 50.56 & 33.02 \\
\hline VA & 5.99 & 3.78 & 5.80 & 4.72 & 54.57 & 37.47 & 55.78 & 37.92 \\
\hline VT & 5.69 & 3.06 & 6.49 & 4.64 & 107.00 & 71.72 & 104.52 & 70.74 \\
\hline WA & 4.90 & 2.68 & 5.72 & 3.36 & 51.51 & 26.77 & 52.08 & 32.11 \\
\hline WI & 4.84 & 3.97 & 6.30 & 5.40 & 59.42 & 39.58 & 60.75 & 40.72 \\
\hline WV & 6.31 & 3.09 & 6.68 & 4.83 & 55.08 & 37.95 & 55.18 & 37.59 \\
\hline WY & 4.38 & 3.30 & 5.03 & 3.76 & 51.43 & 32.65 & 51.70 & 33.11 \\
\hline
\end{tabular}




\subsection{CHP Parameters}

There are a number of available prime movers for CHP systems, ranging from internal combustion engines to microturbines to industrial turbines to fuel cells. Each comes in a variety of sizes, with associated costs and efficiencies. Figure 4 shows an example of energy flows of a combined cooling, heat, and power system. Even for a single size and technology, there are a range of costs and efficiencies. Actual costs are very site-specific; a broad analysis such as this cannot capture the intricacies of installation and operation at any given site. To compensate, we used a range of estimates for each parameter and conducted sensitivity analyses using the minimums and maximums of each parameter.

\section{Combined Cooling, Heat, and Power System}

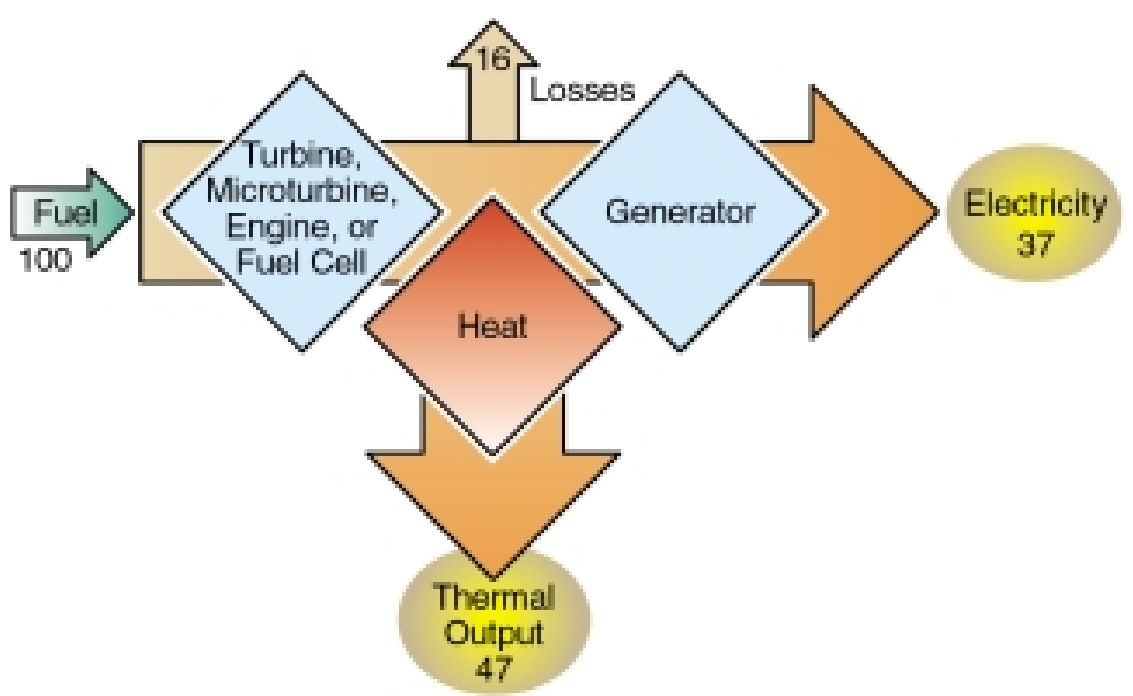

Fig. 4. Energy flows of a combined cooling, heat, and power system.

Resource Dynamics Corporation has conducted a market assessment for CHP (RDC 2001). In the document, they provide estimates of the current and future cost and performance of several technologies and sizes (Table 8). We used the current cost and performance values for reciprocating engines as the base case for this assessment. Engines are the most widely used technology currently; they are low cost and have good load-following capabilities and electrical efficiencies. However, they may have more problems with emissions than the other technologies (turbines and fuel cells). For sensitivity analysis, we used these other technologies and the cost factors that RDC projects for the future. In addition, we ran a sensitivity case with installation costs double that of the current costs in the table. Our experience with installations at federal sites indicates that total project costs can be two to three times those shown in the table. However, those costs include other aspects of the installation not considered in these values, such as refurbished piping, chillers, or other modifications to a site's HVAC system and infrastructure. 
Table 8: CHP cost and operations parameters

\begin{tabular}{|c|c|c|c|c|c|c|c|}
\hline \multirow{2}{*}{ CHP Design } & \multirow[t]{2}{*}{$\begin{array}{l}\text { Size } \\
\text { KW }\end{array}$} & \multicolumn{2}{|c|}{ Installed Cost $\$ / k W$} & \multicolumn{2}{|c|}{$\begin{array}{c}\text { O\&M Cost } \\
\$ / M W h\end{array}$} & \multicolumn{2}{|c|}{ Electrical Efficiency } \\
\hline & & Current & Future & Current & Future & Current & Future \\
\hline Engine $^{a}$ & $45-75$ & 1033 & 815 & 15 & 10 & $31 \%$ & $42 \%$ \\
\hline Engine $^{a}$ & $75-150$ & 953 & 730 & 12 & 9 & $32 \%$ & $42 \%$ \\
\hline Engine $^{a}$ & $150-300$ & 880 & 640 & 12 & 8.5 & $34 \%$ & $43 \%$ \\
\hline Engine $^{a}$ & $300-600$ & 800 & 605 & 10 & 8 & $35 \%$ & $43 \%$ \\
\hline Engine $^{a}$ & $600-1,000$ & 730 & 570 & 8 & 8 & $37 \%$ & $44 \%$ \\
\hline Engine $^{a}$ & $1,000-2,500$ & 704 & 550 & 7.5 & 7.5 & $38 \%$ & $45 \%$ \\
\hline Engine $^{a}$ & $2,500-5,000$ & 622 & 465 & 7.5 & 7.50 & $39 \%$ & $45 \%$ \\
\hline Engine $^{a}$ & $5,000-10,000$ & 575 & 450 & 7 & 7.00 & $42 \%$ & $45 \%$ \\
\hline Engine $^{a}$ & $10,000-20,000$ & 563 & 435 & 7 & 7 & $42 \%$ & $45 \%$ \\
\hline Turbine & $45-75$ & 1383 & 965 & 10 & 10 & $27 \%$ & $40 \%$ \\
\hline Turbine & $75-150$ & 1231 & 860 & 10 & 10 & $27 \%$ & $40 \%$ \\
\hline Turbine & $150-300$ & 1074 & 720 & 9 & 9 & $27 \%$ & $40 \%$ \\
\hline Turbine & $300-600$ & 1015 & 675 & 9 & 9 & $27 \%$ & $40 \%$ \\
\hline Turbine & $600-1,000$ & 757 & 670 & 6 & 6 & $25 \%$ & $40 \%$ \\
\hline Turbine & $1,000-2,500$ & 704 & 525 & 5.5 & 5.5 & $28 \%$ & $40 \%$ \\
\hline Turbine & $2,500-5,000$ & 592 & 420 & 4.5 & 4.5 & $29 \%$ & $40 \%$ \\
\hline Turbine & $5,000-10,000$ & 550 & 400 & 4 & 4 & $31 \%$ & $42 \%$ \\
\hline Turbine & $10,000-20,000$ & 488 & 395 & 4 & 4 & $33 \%$ & $42 \%$ \\
\hline Fuel cell & $150-300$ & 5003 & 1555 & 15 & 15 & $39.6 \%$ & $50 \%$ \\
\hline Fuel cell & $300-600$ & 4812 & 1520 & 15 & 15 & $39.6 \%$ & $50 \%$ \\
\hline
\end{tabular}

${ }^{a}$ The base case used reciprocating engines sized to the average site for the state, with costs and efficiency based on the "current" values above.

Source: RDC 2001, Building Cooling, Heating, and Power (BCHP): A Market Assessment

\subsection{Summary of Parameters}

The key assumptions used to define the base case are summarized in Table 9.

Table 9: Summary of key parameters for base case.

- Federal sites used are from GSA database, focusing on 7 categories with greatest CHP potential and larger facilities. Excluded were housing, storage, "other institutional," or "other" building federal building categories and any buildings where square footage for a category at that site was $<25,000 \mathrm{ft}^{2}$.

- CHP potential was further adjusted to reflect infrastructure compatibility for each type and size of building based on CBECS data (e.g., $88 \%$ of hospital facilities over $100 \mathrm{ft}^{2}$ were assumed to have gas available and compatible heat distribution systems, while only $48 \%$ of office facilities in that size range were considered compatible).

$\square$ Energy intensities for each category and geographic area are from CBECS database, with industrial building types using laboratory intensities.

․ Energy prices were based on 2000 Industrial energy tariffs for each state from ElA.

a CHP is assumed to provide $75 \%$ of electrical needs of hospitals, prisons, and industrial facilities. It provides $50 \%$ of electrical needs of offices, schools, R\&D and service facilities.

- Hospitals, prisons, and industrial facilities are assumed to have load factors (average to peak production) of $85 \%$; load factors for offices, schools, R\&D and service facilities are $35 \%$.

- CHP uses reciprocating engine technologies using industry citations to estimate current costs and efficiency, with electrical efficiencies based on the unit's size and a recoverable waste heat efficiency of $75 \%$; steam boilers replaced by CHP are assumed to have $80 \%$ efficiency.

a Hospitals, prisons, and industrial facilities install two CHP units to provide increased reliability while others only install one.

a All recoverable waste heat is assumed utilized by the facility to offset thermal energy purchases (this assumption is examined in sensitivity analyses).

a Only sites where the simple payback period was 10 years or less were counted in base case. 
This page intentionally left blank. 


\section{CHP Calculation}

Based on the data discussed in Sect. 2, we could calculate the amount of CHP that could be installed. For each state, we summed the amount of federal floor space of each building type. Table 10 shows the methodology used to calculate the CHP potential capacity for each state, using California as an example. The following sections walk the reader through the calculations in Table 10 row by row, referencing data sources as appropriate.

We summed the space of those sites that had more than $100,000 \mathrm{ft}^{2}$ of any given building type (row a); in California there were 19 sites with hospitals (row b). The electric intensity for hospitals in the Pacific region was $101 \mathrm{kBtu} / \mathrm{ft}^{2}$ (row c) (Table 2). Based on the CBECS analysis, 88\% of hospital facilities over $100,000 \mathrm{ft}^{2}$ in size are estimated to have infrastructure compatible with CHP (row d) (Table 6).

Table 10: CHP capacity calculation, example for California buildings $>100,000 \mathrm{ft}^{2}$

\begin{tabular}{|c|c|c|c|c|c|c|c|c|}
\hline & $\begin{array}{l}\bar{\pi} \\
\frac{\pi}{0} \\
\text { 음 } \\
\text { 우 }\end{array}$ & 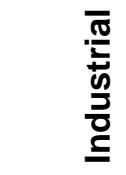 & $\frac{d}{0}$ & 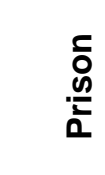 & $\underset{\sim}{\stackrel{\varnothing}{\not}}$ & $\begin{array}{l}\overline{8} \\
\stackrel{0}{0} \\
\bar{\delta}\end{array}$ & 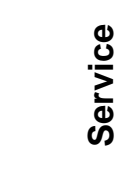 & $\begin{array}{l}\bar{\sigma} \\
\text { 0 } \\
\end{array}$ \\
\hline (a)Total area, $\mathrm{kft}^{2 a}$ & 11,917 & 6,290 & 50,371 & 3,609 & 23,931 & 11,814 & 72,547 & $365,154^{b}$ \\
\hline (b)Total no. of sites ${ }^{a}$ & 19 & 9 & 133 & 10 & 28 & 39 & 90 & $551^{b}$ \\
\hline (c)Intensity kBtu/ft ${ }^{2}$ & 101 & 111 & 104 & 71 & 111 & 32 & 59 & \\
\hline (d) $\%$ bldgs. w/piping & $88 \%$ & $78 \%$ & $48 \%$ & $56 \%$ & $78 \%$ & $71 \%$ & $24 \%$ & \\
\hline (e) \% electric provided & $85 \%$ & $85 \%$ & $50 \%$ & $85 \%$ & $50 \%$ & $50 \%$ & $50 \%$ & \\
\hline (f) GBtu of electricity & 901 & 462 & 1,254 & 121 & 1,033 & 134 & 514 & 4,421 \\
\hline (g) GWh of electricity & 264 & 135 & 368 & 36 & 303 & 39 & 151 & 1,296 \\
\hline (h) Load factor & $75 \%$ & $75 \%$ & $35 \%$ & $75 \%$ & $35 \%$ & $35 \%$ & $35 \%$ & \\
\hline (i) Capacity, MW & 40.2 & 20.6 & 119.9 & 5.4 & 98.8 & 12.8 & 49.1 & 346.9 \\
\hline (j) CHP units per facility & 2 & 2 & 1 & 2 & 1 & 1 & 1 & \\
\hline (k) Avg. capacity, MW & 1.20 & 1.48 & 1.89 & 0.49 & 4.55 & 0.47 & 2.27 & \\
\hline (I) $\mathrm{CHP}$ elec. efficiency ${ }^{c}$ & $38 \%$ & $38 \%$ & $38 \%$ & $35 \%$ & $39 \%$ & $35 \%$ & $38 \%$ & \\
\hline (m) Equipment cost, $\mathrm{k} \$$ & 28,311 & 14,503 & 84,399 & 4,327 & 61,447 & 10,272 & 34,599 & 237,859 \\
\hline (n) Operating cost, $\mathrm{k} \$$ & 1,982 & 1,015 & 2,757 & 355 & 2,272 & 394 & 1,130 & 9,904 \\
\hline (o) Gas costs, k\$ & 5,727 & 2,934 & 7,967 & 780 & 6,540 & 864 & 3,266 & 28,078 \\
\hline (p) Electric savings, $k \$$ & 14,242 & 7,296 & 19,814 & 1,916 & 16,327 & 2,122 & 8,123 & 69,840 \\
\hline (q) Net savings, $\mathrm{k} \$$ & 6,534 & 3,347 & 9,090 & 780 & 7,516 & 864 & 3,726 & 31,858 \\
\hline (r) Payback, years & 4.3 & 4.3 & 9.3 & 5.5 & 8.2 & 11.9 & 9.3 & \\
\hline (s) Energy saving, Gbtu & 1,740 & 891 & 2,421 & 232 & 1,999 & 257 & 992 & 8,534 \\
\hline
\end{tabular}

${ }^{a}$ Includes all buildings in GSA database $>100,000 \mathrm{ft}^{2}$, including those without CHP potential

${ }^{b}$ Total includes other building categories not shown

${ }^{c}$ CHP system efficiency for converting fuel to electricity (from Table 8). This does not reflect overall system efficiency that would include use of waste heat for thermal applications.

The amount of energy provided by CHP (row e) and the load factor of the CHP units (row h) are dependent on the profile of energy used by the building being modeled. Two basic load curves for implementation of CHP were developed: one for a system used during typical 5-day-per-week office building occupation, and one for a more continuously run, load-following system. 
The first diagram (Fig. 5) shows the electric load profile of a typical, large office building (ORNL 1994). While power demands peak at $1.7 \mathrm{MW}$, during nights and weekends they drop to around $0.2 \mathrm{MW}$, giving an average load of around $0.6 \mathrm{MW}$. In this system, a $0.8 \mathrm{MW}$ CHP unit would operate during the hours of 7 a.m. to 7 p.m. on weekdays (with some adjustment for start-up). This would replace power purchases during the most expensive peak times of the day, and would present a relatively flat load to the utility, further improving the likelihood of low power rates. In this example, CHP provides $50 \%$ of the electric energy needs of the building and has a capacity factor of $35 \%$.

Fig. 5: Load curve template for office building and CHP unit used during occupation.

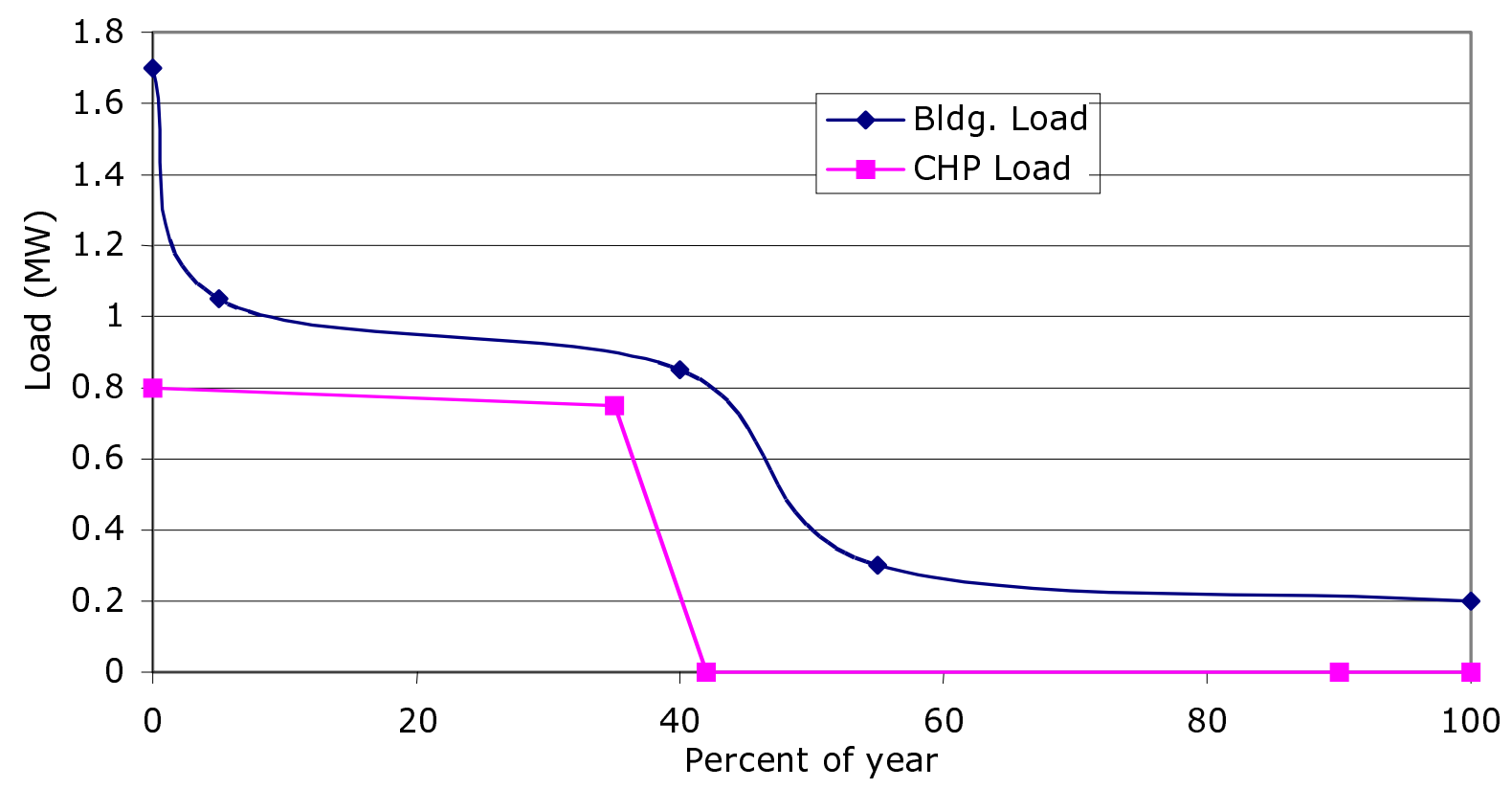

An alternative system would be for a building that operates relatively constantly (has a high load factor) and uses CHP to provide a large share of its steam and electricity needs. The example is from a system installed at the Iowa Methodist Medical Center. This hospital's power usage peaks at around 3.6 MW, with a minimum of 1.8 MW. They chose to install two 1.5-MW dual-fuel diesel generators with heat recovery.

Figure 6 shows the average power levels for the hospital for each of three shifts for each month of the year. Ordering them from highest to lowest shows the load curve, the fraction of year when demand is at or above a certain load. If two CHP units are installed, each capable of providing 1.5 MW, they could provide the amounts of energy shown by operating at an average capacity factor of $77 \%$. Combined, they provide $87 \%$ of the hospital's electricity needs. At low power levels, one unit would operate at full power. Either could be operating, with the other one down for maintenance. At times of highest demand, both would operate, providing $3 \mathrm{MW}$ of power. In between, they could either both be partly loaded, or one could operate at full load while the other operates at partial load. 
Fig. 6: Load curve template for hospital with two load-following CHP units.

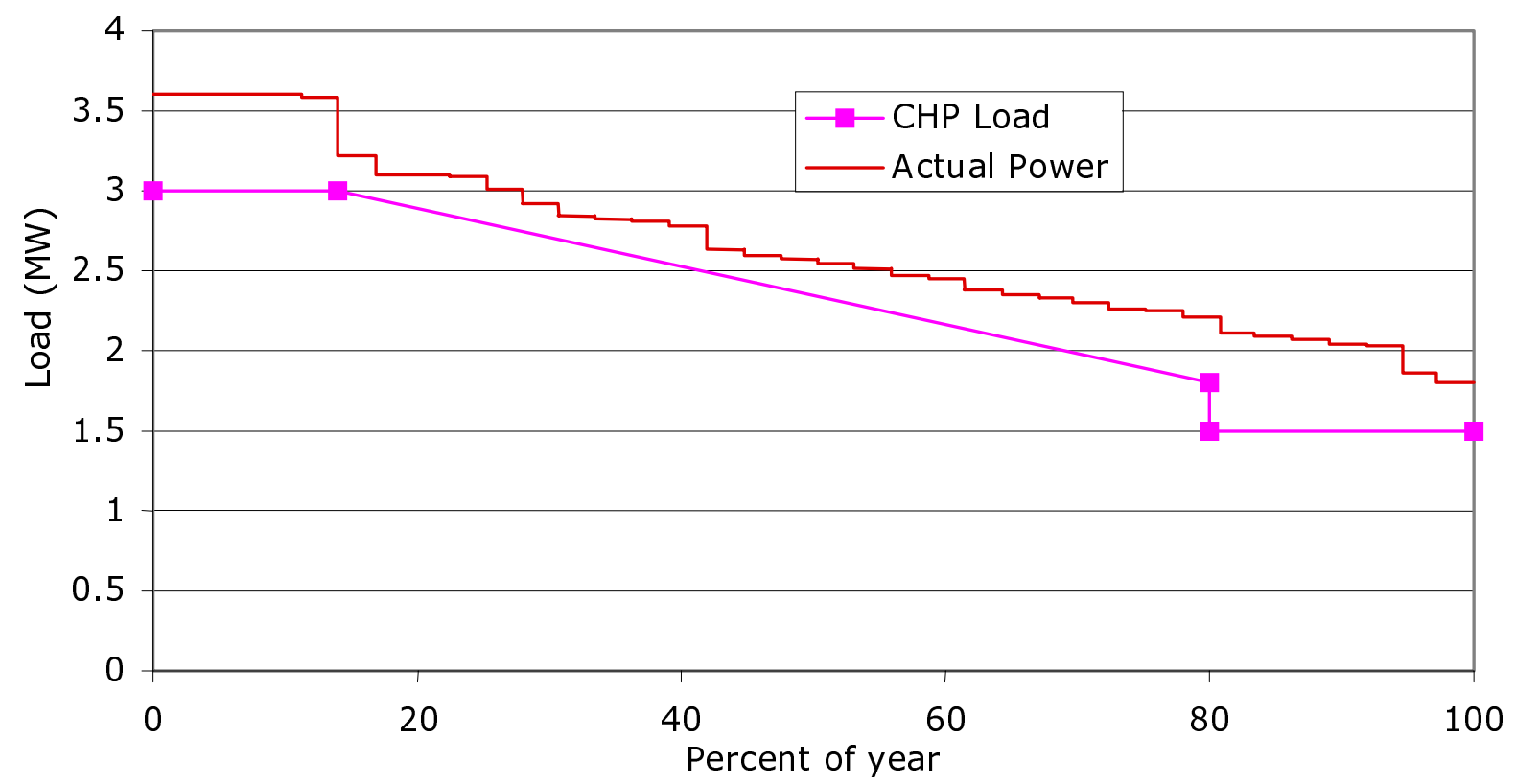

In Fig. 7, the hospital's steam needs did not match its electricity needs. Steam requirements were highest in winter months, when electricity needs were lower. To compensate, the hospital installed supplementary firing capability.

Fig. 7. Comparison of electricity and steam needs for Iowa Methodist Medical Center

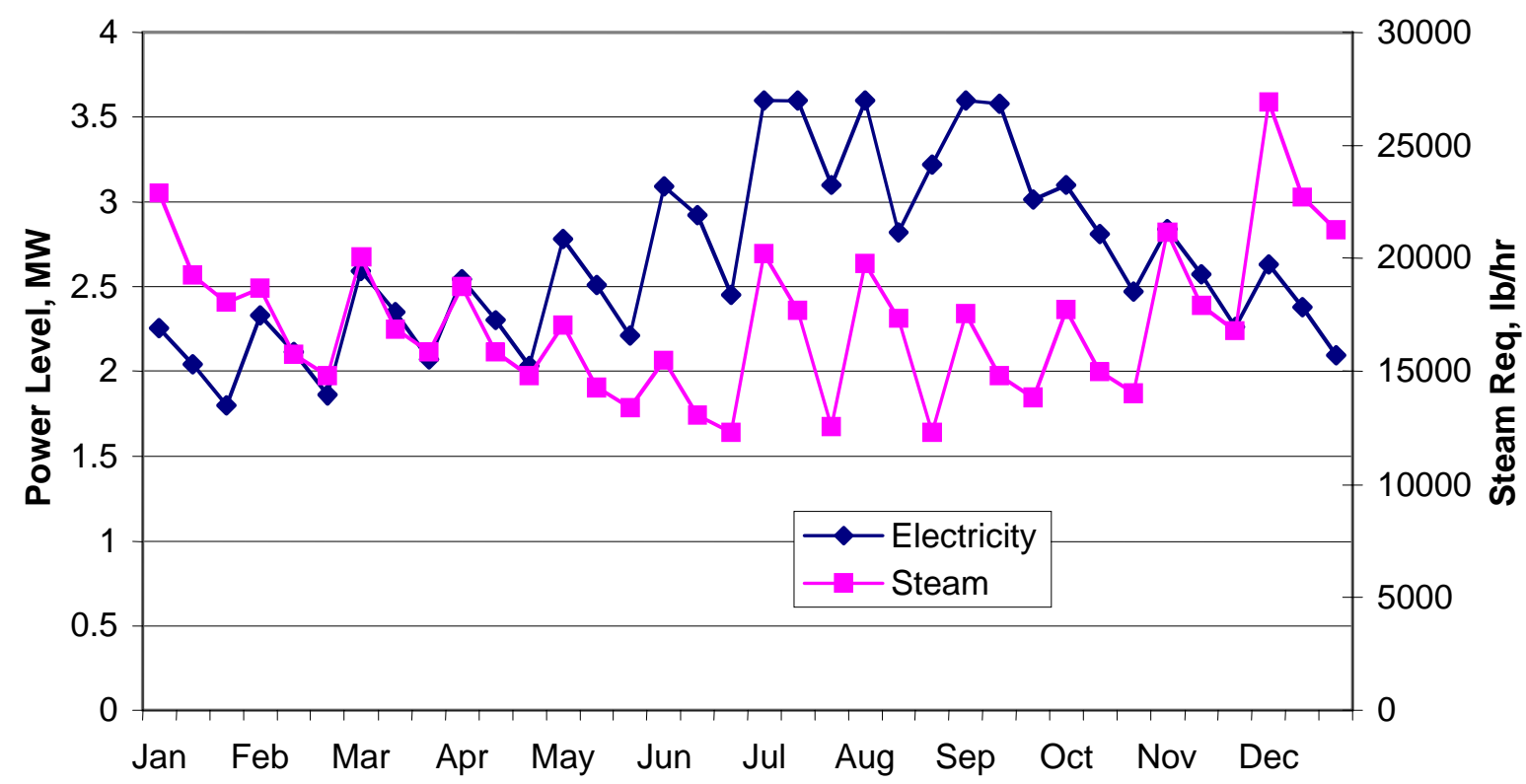


For the present analysis, offices, schools, $R \& D$, and service buildings were assumed to have building load profiles similar to the first example, and a single unit CHP system is sized to provide $50 \%$ of the electric energy needs at a capacity factor of $35 \%$. Hospitals, prisons, and industrial facilities used the parameters from the second example: $85 \%$ of electricity provided and a capacity factor of $75 \%$.

It is important to stress that the design of a CHP system is highly site-specific, and is typically sized to fit a thermal load curve and to operate close to full power to the maximum degree possible (70-99\% of the time). Sizing CHP systems to thermal loads was done as a sensitivity test in this model. That scenario is based only on heating loads and generated a total capacity slightly below the base case. Since CHP systems can also support cooling, dehumidification, and other applications, the estimate of CHP capacity sized to thermal demand must look at the timing, duration and other specifications for all the potential waste heat applications at a given site.

Returning to the California example of CHP capacity calculations in Table 10, the percentage of electricity provided by the CHP system is shown in row e, and the load factor of the CHP is shown in row h. Using the energy intensities for each building type and state, we calculated the amount of electricity needed by those sites. The letters in parentheses designate the respective rows in Table 10.

(f) GBtu electricity $=\left(\right.$ a) total $\mathrm{ft}^{2} *$ (c) electric intensity $*(\mathrm{~d}) \%$ with piping $*$ (e) $\%$ electric $/ 1000$

(g) GWh electricity = (f) GBtu of electricity / 3.412 Btu/Wh

(i) Capacity, MW = (g) GWh of electricity / 8760 h / (h) load factor

Dividing the total capacity for the state by the number of sites (row b) and number of units per site (row $\mathrm{j}$ ) gave the average capacity per unit (row k). For the load-following building types (hospitals, industrial, and prisons), we assumed that two equal sized units would be installed at a site. The corresponding CHP project efficiency (row 1), equipment costs (row m), and O\&M cost (row n) were calculated using information from Table 10 times the CHP total capacity (row i) and production (row $\mathrm{g}$ ).

The amount of gas needed is the amount to make the electricity based on the CHP efficiency minus the amount of gas displaced by the CHP thermal exhaust. A limit can be placed on the amount of exhaust gas that can be used so that it does not exceed some fraction of the thermal energy demand (that was estimated based on the CBECS gas intensity data and the square feet of the facility). Allowance was made for the relative efficiency of a boiler (80\%) versus the lower thermal efficiency of CHP (75\%) due to extra thermal losses.

Gas exhaust used = minimum [(f) GBtu electricity / (1) efficiency - GBtu electricity, input fraction of thermal energy needs calculated using gas intensity]

o) Gas cost $=[(\mathrm{f})$ GBtu electricity / (1) efficiency - gas exhaust used $* 75 \% / 80 \%] *$ gas price $/ 1000$

Electricity savings are based on the amount of electricity created by the CHP times the average state price used (Table 7). In the base case, we used the industrial price from 2000. Net savings are then calculated by subtracting the cost of gas and the operating cost of the CHP system. The simple payback for the system is then the capital cost of the project divided by the annual savings.

(p) electricity savings $=(\mathrm{g}) \mathrm{GWh}$ of electricity $*$ electricity price $/ 1000$ 
(q) net savings $=(\mathrm{p})$ electric savings $-(\mathrm{n})$ operating costs $-(\mathrm{o})$ gas costs

(r) payback $=(\mathrm{m})$ equipment cost $/(\mathrm{q})$ net savings

Note that in the example calculation for California (Table 10), the office category is shown to have a surprisingly high capacity (120 MW), but the payback is very near the 10-year limit. Thus, this capacity is marginal at present and very sensitive to small changes in assumptions (e.g., capacity factor, percentage of energy from CHP, electric intensity, and ability to use recovered heat), which could reduce this capacity to zero under the base case minimum pay back criteria. Also, note that the "schools" category for California has an average payback period of 12 years. Thus in Table 14, the analysis shows 0 MW of CHP potential for schools in California (they did not fall under the 10-year payback minimum). This highlights one of the limitations of using statewide averages, but without working with more detailed information on the specific sites, the authors believe that a more detailed analysis would simply generate a false appearance of precision.

Separate calculations were made for those buildings between $25,000 \mathrm{ft}^{2}$ and $100,000 \mathrm{ft}^{2}$ using the data sets for different energy intensities and different percentages of buildings with HVAC systems conducive to CHP. These smaller facilities offer little CHP potential under the base case assumptions (10 MW).

Simple paybacks do not include the "time value" of money (changing value of money due to interest and inflation). They just show in simple terms how long it would take for the initial investment to be recovered. Including the real cost of money (interest rate minus inflation) raises the number of years to payback, depending on the assumed cost and the number of years (Fig. 8). Note that with a simple payback of 10 years and a real cost of money of $6 \%$, the actual payback is closer to 15 years, and a 15year simple payback rises past 40 years.

Fig. 8: Effect of including cost of money in payback calculation.

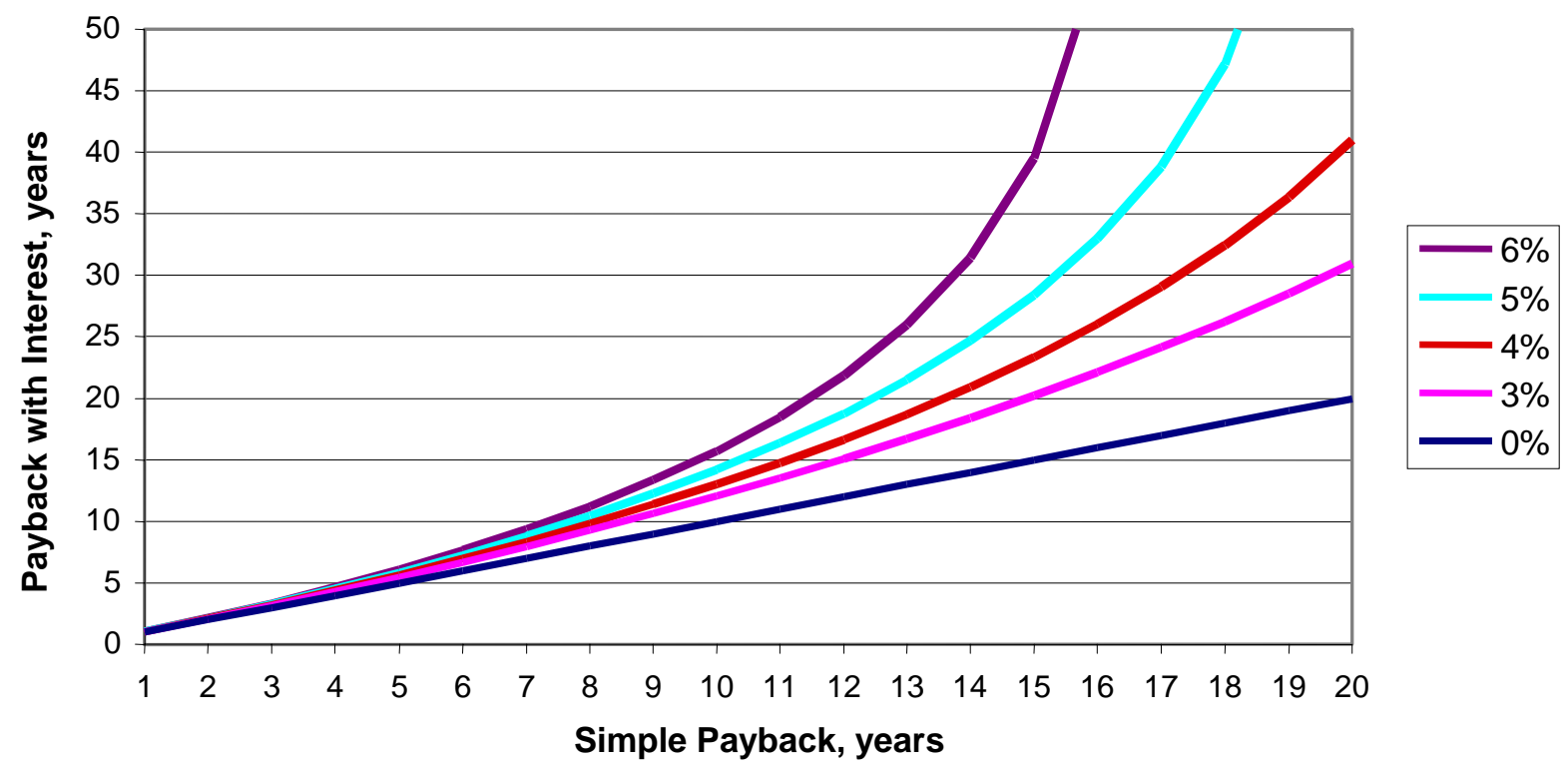


This page intentionally left blank. 


\section{CHP Results}

\subsection{Potential Capacity}

Using a cutoff of 10 years for simple payback and the set of base case parameters discussed earlier (Table 9), the total amount of CHP potential capacity for federal facilities nationwide is estimated to be between 1500 and $1600 \mathrm{MW}$ (Table 11). Under the operating assumptions of the base case, the CHP systems would produce 7.7 TWh of electricity representing over $13 \%$ of the 57 TWh total electricity purchased by the federal government in FY 2000 (FEMP 2002). This CHP capacity would provide electricity and thermal energy for about $580 \mathrm{M} \mathrm{ft}^{2}$ of building space in $9 \%$ of all federal sites. The potential will be greatest in large sites with central plants or mechanical rooms and high electricity rates. These CHP capacity numbers are based on the set of base case assumptions discussed thus far:

- using reciprocating gas engines at their current estimated cost and efficiencies,

- energy prices at 2000 industrial rates for each state,

- covering $75 \%$ or $50 \%$ of estimated electric demand with load factors at $85 \%$ or $35 \%$, depending on building type and size

- considering only the percentage of CHP-compatible federal facilities calculated from the categories of Table 6 with 25,000 $\mathrm{ft}^{2}$ or more,

- assuming all recoverable waste heat is utilized by the site, and

- with a simple payback less than ten years.

Changing these parameters can give widely different amounts of CHP potential and energy savings.

Table 11: National CHP potential by building category at federal facilities using base case assumptions

\begin{tabular}{|c|c|c|c|c|c|c|c|c|}
\hline & 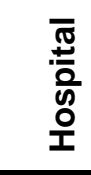 & 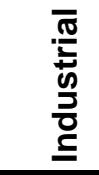 & $\frac{0}{0}$ & $\begin{array}{l}\frac{c}{0} \\
\frac{\leftrightarrow}{2} \\
\frac{2}{2}\end{array}$ & $\underset{\not}{\not}$ & $\begin{array}{l}\overline{0} \\
\frac{0}{0} \\
\dot{c}\end{array}$ & 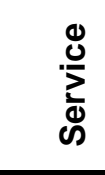 & 흄 \\
\hline Total $\mathrm{Mft}^{2}$, all buildings ${ }^{\mathrm{a}}$ & 141 & 115 & 514 & 41 & 144 & 136 & 463 & $2757^{b}$ \\
\hline $\begin{array}{l}\mathrm{Mft}^{2} \text { buildings with } \mathrm{CHP} \\
\text { payback }<10 \text { years }\end{array}$ & 113 & 80 & 146 & 16 & 100 & 42 & 82 & 579 \\
\hline Total number of sites ${ }^{a}$ & 331 & 181 & 2302 & 99 & 421 & 917 & 1033 & $8182^{b}$ \\
\hline $\begin{array}{l}\text { Number of sites with CHP } \\
\text { payback }<10 \text { years }\end{array}$ & 235 & 75 & 167 & 38 & 70 & 42 & 74 & 700 \\
\hline$\%$ of sites with CHP potential & 71 & 42 & 7 & 38 & 17 & 5 & 7 & 9 \\
\hline $\begin{array}{l}\text { Potential TWh of electricity } \\
\text { from } \mathrm{CHP}\end{array}$ & 2.93 & 2.25 & 0.76 & 0.24 & 0.81 & 0.06 & 0.65 & 7.69 \\
\hline Potential CHP capacity, MW & 446 & 342 & 248 & 36 & 265 & 18 & 211 & 1567 \\
\hline
\end{tabular}

${ }^{a}$ Includes buildings in GSA database $>25,000 \mathrm{ft}^{2}$, even those without CHP potential

${ }^{\mathrm{b}}$ Total includes other building types not shown

Under the base case, federal hospitals have the highest potential for CHP at $446 \mathrm{MW}$. Hospitals also show the most promising target of opportunity, since $71 \%$ of their sites over $25,000 \mathrm{ft}^{2}$ are estimated to have 
potential (Fig. 9). Hospital numbers may be limited partly by the assumption that only $66 \%-88 \%$ of facilities have fuel and HVAC systems compatible with CHP (experience suggests that this is a conservative number). Industrial buildings are next in potential capacity, at $342 \mathrm{MW}$, and are also second in percentage of sites at $42 \%$. Since these two categories were modeled using the 24/7 load-following CHP assumed to provide $85 \%$ of the facility's electricity at a relatively high capacity factor (75\%), they provide more than two-thirds of the total electricity and potential savings estimated by this model.

Fig. 9. Percent of federal sites with CHP potential by building category and corresponding capacity (MW).

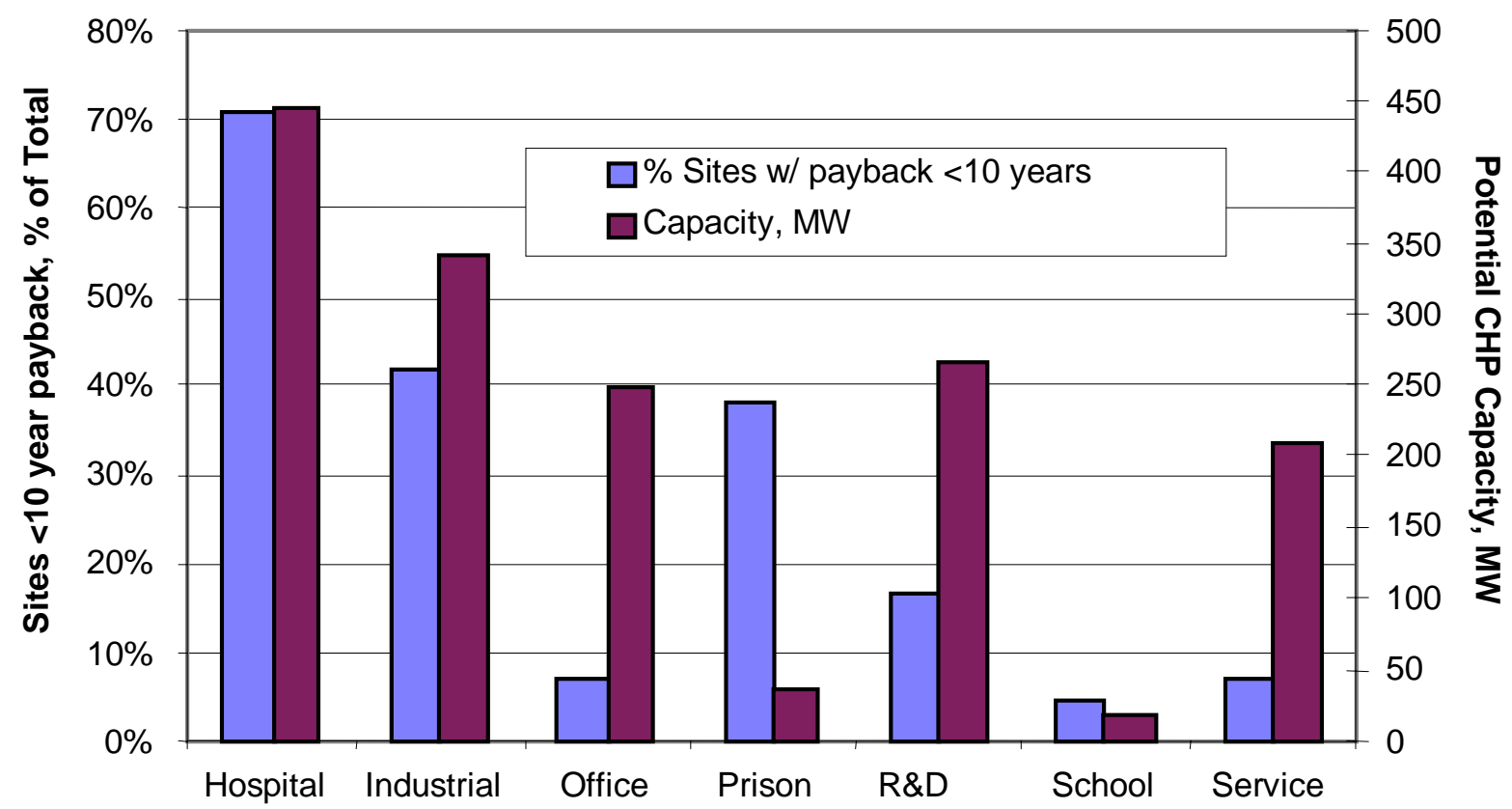

R\&D facilities, office buildings, and service buildings provide similar amounts of capacity $(265,248$, and $211 \mathrm{MW}$, respectively) under the base case. These three categories were modeled in the base case as using the weekday occupation load curve (CHP provides 50\% of electricity at a $35 \%$ capacity factor) rather than the 24/7 load-following CHP profile. Some R\&D and service facilities may be more appropriately modeled using the higher load curve similar to hospitals or industrial sites. Under that alternative load profile, R\&D CHP capacity increases from 265 to 386 MW (45\%). Service buildings under the alternative load scenario increase by a modest $10 \%$ to $233 \mathrm{MW}$.

The amount of CHP capacity estimated available in federal office buildings appears high compared with experience at federal facilities. First, it should be noted that the office category is nearly five times larger than most other building categories. Second, under the base case scenario, only $7 \%$ of large federal office facilities show potential for CHP. In the case of offices, we believe that this is still an optimistic estimate due to the assumptions. The base case assumption that all of the recoverable heat from the CHP system can be applied to off-set thermal energy needs at the site is more tenuous for office buildings than for other categories. Full exhaust heat utilization would often be more difficult or costly due to the typical location and load profiles for office buildings as compared to hospitals or industry. If the use of recoverable heat were limited to the amount estimated by CBECS based on the average gas intensity for office buildings, then the potential CHP capacity for federal offices would fall from $248 \mathrm{MW}$ to $49 \mathrm{MW}$. Furthermore, typical federal office buildings will present more obstacles to retrofitting CHP than other 
large facilities with existing central plants and boilers. Our assessment accounted for this using the "CHP compatible" factor in Table 6.

Another category with very high total floor space was service facilities. But CHP capacity is limited due to a low average energy intensity and lower percentage of building HVAC and distribution assumed to be compatible with CHP. Thus, service was fifth in potential capacity. Schools and prisons ranked relatively low in potential capacity, due both to relatively low floor space and energy intensities. A relatively high proportion of prisons (38\%) show potential, so even though this may be a relatively small niche, there is a good likelihood of acceptability at those sites. Also, the methodology's data and assumptions for prison compatibility with CHP and energy intensity may be overly conservative.

Table 12 shows the investment cost, annual operating costs, and energy savings expected if all the CHP identified in the base case were implemented at federal sites. There are one-time installation, annual operating, and annual gas purchase costs. Savings come from reduced electricity purchases, and the net annual savings are these savings less annual costs. Simple payback is then just the installation cost divided by the net savings, to show the number of years until the installation cost is recovered. The payback numbers reflect national averages for each building category.

Table 12: CHP costs, savings, and payback, by building category, under base case assumptions

\begin{tabular}{|c|c|c|c|c|c|c|c|c|}
\hline & $\begin{array}{l}\bar{\pi} \\
\frac{\pi}{\overline{0}} \\
\text { 0 } \\
\text { 우 }\end{array}$ & 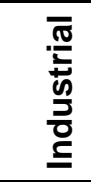 & $\underbrace{\stackrel{0}{0}}_{\frac{0}{0}}$ & 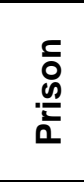 & $\underset{\sim}{\stackrel{\not}{\not}}$ & $\begin{array}{l}\overline{0} \\
\frac{0}{0} \\
\text { ल }\end{array}$ & 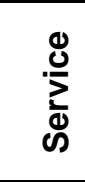 & $\begin{array}{l}\bar{\pi} \\
\stackrel{0}{0}\end{array}$ \\
\hline Capacity, MW & 446 & 342 & 248 & 36 & 265 & 18 & 211 & 1567 \\
\hline Installation cost, $\mathrm{M} \$$ & 319 & 222 & 174 & 28 & 163 & 14 & 135 & 1055 \\
\hline Operating cost, $\mathrm{M} \$$ & 23 & 17 & 6 & 2 & 6 & 0 & 5 & 59 \\
\hline Gas costs, $M \$$ & 55 & 42 & 15 & 4 & 16 & 1 & 12 & 145 \\
\hline Electricity savings, $\mathrm{M} \$$ & 138 & 100 & 44 & 11 & 44 & 3 & 35 & 375 \\
\hline Net annual savings, $\mathrm{M} \$$ & 60 & 41 & 23 & 5 & 22 & 2 & 18 & 171 \\
\hline Average payback, years & 5.3 & 5.5 & 7.5 & 5.8 & 7.4 & 7.5 & 7.4 & 6.2 \\
\hline
\end{tabular}

A CHP system is generally not more efficient at producing electricity alone than the central grid, and properly maintained boilers can be more efficient at producing thermal energy alone than a CHP system. But the combined generation of electricity and thermal energy on-site by a well-designed CHP system is more efficient overall than the combined efficiencies of these two alternatives. One key to ensuring an efficient CHP system is to maximize the use of thermal energy (waste heat) from the generation process. This may mean that the most economic system does not necessarily have the highest electrical efficiency. If the building requires a high amount of thermal energy (e.g., a commercial laundry or industrial foodprocessing plant), then the most economic system might be one with slightly lower electrical efficiency but with a larger amount of recoverable, high-temperature exhaust. Emissions or other site-specific factors may also override electrical efficiency when determining which CHP system best meets a facility's needs.

Because CHP uses energy to generate electricity on site, and because it is slightly less efficient for thermal purposes than a regular boiler, the energy use at the site will increase with a CHP system, and site-based energy savings will be negative. However, since losses associated with generating and distributing the electricity (from the alternate central source) will be avoided, CHP results in a net savings of primary (source) energy. Table 13 estimates the amount of source energy savings for each building type. The additional gas used at the site is higher in Btu value than the electricity generated on site. 
However, using an average heat rate for central power generation of 10,346 Btu/kWh (FEMP 2001), the energy losses at the central generating plant avoided by CHP more than compensate for the extra gas used, giving a significant net primary energy savings when comparing site to source. The estimated annual source-based energy savings that would accrue if all $1.57 \mathrm{GW}$ of CHP were implemented under the base case is 50.7 trillion Btu. This represents about $8 \%$ of total primary energy consumption reported for federal buildings and facilities in 1999 (FEMP 2001).

Table 13: Site and source energy savings from federal CHP, TBtu/year

\begin{tabular}{|c|c|c|c|c|c|c|c|c|}
\hline & $\begin{array}{l}\bar{\pi} \\
\frac{\pi}{\overline{0}} \\
\text { क } \\
\text { 운 }\end{array}$ & 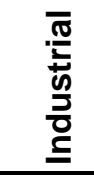 & 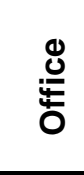 & 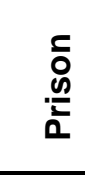 & $\underset{\sim}{\underset{\sim}{\not}}$ & $\begin{array}{l}\overline{8} \\
\text { ¿ } \\
\bar{\delta}\end{array}$ & 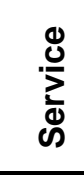 & $\begin{array}{l}\bar{\pi} \\
\stackrel{0}{0}\end{array}$ \\
\hline Additional gas use at site & 11.0 & 8.4 & 2.9 & 0.9 & 3.0 & 0.2 & 2.4 & 28.9 \\
\hline Avoided electricity purchases & 10.0 & 7.7 & 2.6 & 0.8 & 2.8 & 0.2 & 2.2 & 26.2 \\
\hline Site energy savings & -1.0 & -0.8 & -0.3 & -0.1 & -0.3 & 0.0 & -0.2 & -2.7 \\
\hline Avoided source energy use & 30.3 & 23.3 & 7.9 & 2.4 & 8.4 & 0.6 & 6.7 & 79.6 \\
\hline Source energy savings & 19.3 & 14.8 & 5.0 & 1.5 & 5.4 & 0.4 & 4.3 & 50.7 \\
\hline
\end{tabular}

\subsection{CHP Potential by State}

Under base case assumptions, the six states with the largest federal CHP potential are California, Texas, Florida, New Mexico, Colorado, and Tennessee (Table 14). Figure 10 shows the breakdown between building types for the top 20 states. As discussed earlier, California had high values for offices and for R\&D facilities. As shown in Table 10, these are driven both by large numbers of buildings and the low capacity factor for CHP in these building types. The payback is close to ten years, so the projects are more difficult to justify economically than the hospitals or industrial facilities.

Fig. 10: CHP potential capacity by building type for top 20 states, MW.

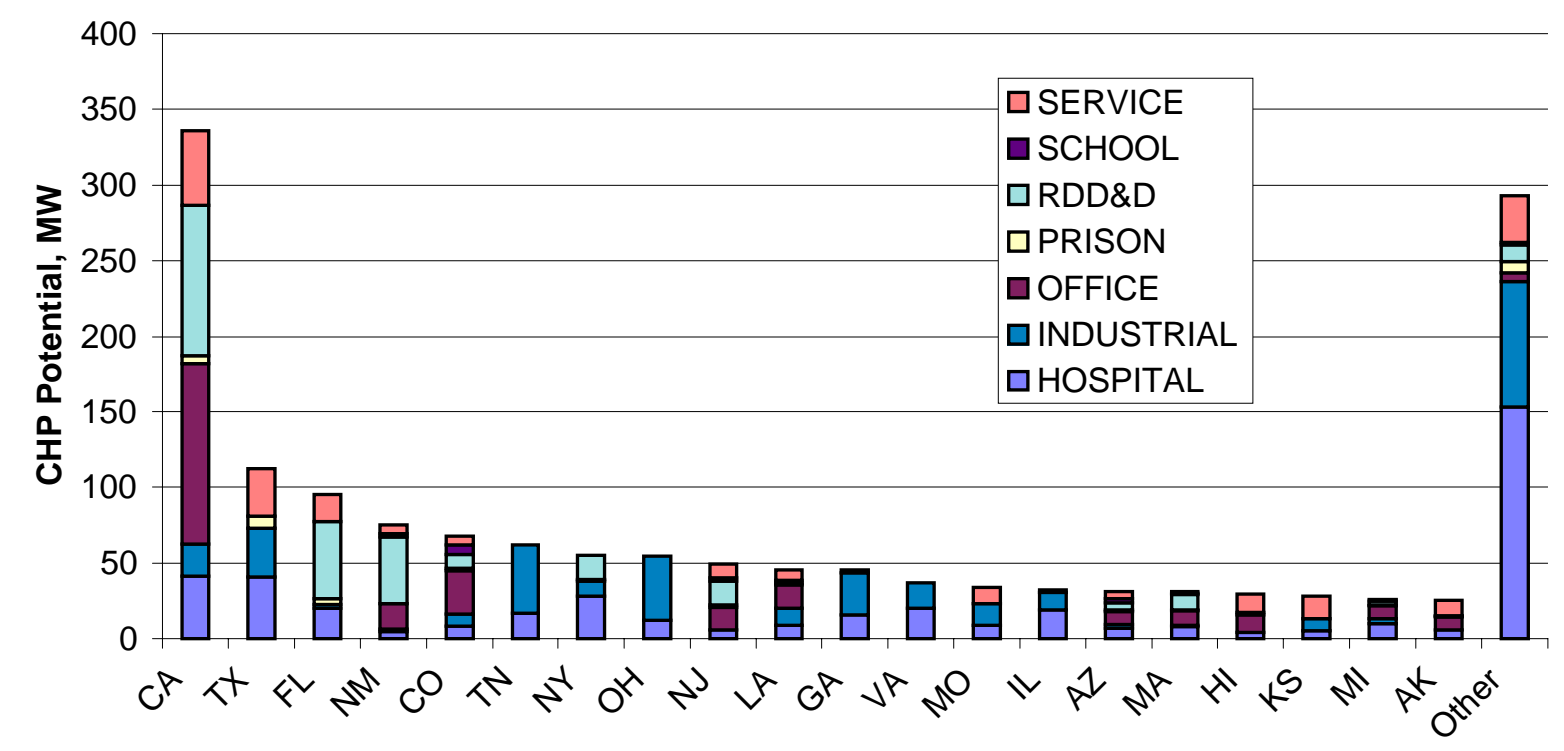


Table 14: State CHP potential capacity by building type under base case, MW

\begin{tabular}{|c|c|c|c|c|c|c|c|c|}
\hline & Hospital & Industrial & Office & Prison & R\&D & School & Service & Total \\
\hline CA & 42 & 21 & 120 & 5 & 99 & - & 49 & 336 \\
\hline $\mathrm{TX}$ & 41 & 32 & - & 8 & - & - & 31 & 112 \\
\hline FL & 20 & 3 & - & 4 & 51 & - & 18 & 96 \\
\hline NM & 5 & 2 & 17 & - & 44 & 2 & 6 & 75 \\
\hline CO & 8 & 8 & 29 & 2 & 9 & 6 & 6 & 68 \\
\hline TN & 17 & 45 & - & 0 & - & - & - & 62 \\
\hline NY & 28 & 10 & - & 1 & 16 & - & - & 55 \\
\hline $\mathrm{OH}$ & 12 & 42 & - & - & - & - & - & 54 \\
\hline NJ & 6 & - & 15 & 1 & 16 & 2 & 9 & 49 \\
\hline LA & 9 & 11 & 16 & 1 & 2 & - & 7 & 46 \\
\hline GA & 16 & 27 & - & 2 & - & - & - & 46 \\
\hline VA & 20 & 17 & - & - & - & - & - & 37 \\
\hline MO & 9 & 15 & - & - & - & - & 10 & 34 \\
\hline IL & 19 & 11 & - & 2 & - & - & - & 33 \\
\hline AZ & 7 & 3 & 8 & 1 & 5 & 3 & 5 & 31 \\
\hline MA & 8 & 1 & 9 & 1 & 10 & 1 & 1 & 31 \\
\hline HI & 4 & 0 & 12 & - & 0 & 1 & 12 & 30 \\
\hline KS & 5 & 8 & - & 0 & - & - & 14 & 28 \\
\hline MI & 10 & 3 & 8 & 0 & 3 & - & 1 & 26 \\
\hline AK & 6 & - & 9 & - & 0 & 1 & 10 & 25 \\
\hline PA & 17 & 4 & - & 2 & - & - & - & 24 \\
\hline DC & 16 & 7 & - & - & - & - & - & 23 \\
\hline MN & 6 & 11 & - & 0 & - & - & 3 & 21 \\
\hline WA & 12 & 7 & - & - & - & - & - & 19 \\
\hline IN & 7 & 10 & - & 1 & - & - & - & 18 \\
\hline AL & 16 & 2 & - & - & - & - & - & 18 \\
\hline OK & 6 & 11 & - & 1 & - & - & - & 18 \\
\hline AR & 10 & 1 & - & 1 & 2 & - & 3 & 18 \\
\hline WI & 9 & 8 & - & - & - & - & - & 17 \\
\hline NC & 14 & 2 & - & 1 & - & - & - & 17 \\
\hline SC & - & 13 & - & - & - & - & - & 13 \\
\hline ND & 2 & - & - & - & - & - & 11 & 13 \\
\hline MS & 9 & 2 & - & 0 & - & - & - & 12 \\
\hline CT & 3 & 2 & 3 & 0 & 3 & 0 & 0 & 11 \\
\hline SD & 4 & - & - & - & - & - & 8 & 11 \\
\hline NV & 2 & 2 & - & 0 & - & - & 4 & 9 \\
\hline RI & 1 & - & 2 & - & 5 & 1 & 1 & 8 \\
\hline WV & 8 & - & - & - & - & - & - & 8 \\
\hline OR & 5 & - & - & - & - & - & - & 5 \\
\hline IA & 4 & - & - & - & - & - & - & 4 \\
\hline NH & 1 & - & 1 & - & 1 & 0 & 1 & 4 \\
\hline ME & 1 & - & - & - & - & - & - & 1 \\
\hline VT & 1 & - & 0 & - & 0 & 0 & 0 & 1 \\
\hline DE & - & - & - & - & - & - & - & - \\
\hline ID & - & - & - & - & - & - & - & - \\
\hline KY & - & - & - & - & - & - & - & - \\
\hline MD & - & - & - & - & - & - & - & - \\
\hline MT & - & - & - & - & - & - & - & - \\
\hline NE & - & - & - & - & - & - & - & - \\
\hline UT & - & - & - & - & - & - & - & - \\
\hline WY & - & - & - & - & - & - & - & - \\
\hline Total & 446 & 342 & 248 & 36 & 265 & 18 & 211 & 1,567 \\
\hline
\end{tabular}


Besides the amount of floor space and energy intensity in any state, a key factor is the relative price of natural gas and electricity. States with low gas prices and high electricity prices are the best candidates for CHP. Contrarily, high gas prices and low electricity prices make CHP less attractive. Figure 11 shows the national amount of potential CHP capacity, based on Table 14. Figure 12 shows the states with the highest difference between electricity and gas prices. Note that there is a strong correlation between the two figures. Exceptions exist primarily because states with higher numbers of large federal buildings are more likely to have higher CHP potential.

Some industry personnel have suggested that high gas prices alone provide better economics for CHP. However, this is only true if the price of electricity in the region is tied to the price of gas, thereby increasing the spread in \$/MBtu between the two as gas prices rise. Keeping the spark spread constant while raising the price of gas decreases the value of CHP slightly since it tends to be slightly less efficient in thermal utilization than regular boilers. Higher gas prices alone, without corresponding electricity price increases, reduce the calculated CHP capacity in the model.

CHP and emissions: EPA considers CHP to be a key pollution prevention tool. EPA estimates that electric power generation plants are responsible for:

- $67 \%$ of all emissions of sulfur dioxide $\left(\mathrm{SO}_{2}\right)$, the leading component of acid rain and fine particulates;

- $40 \%$ of all man-made emissions of carbon dioxide $\left(\mathrm{CO}_{2}\right)$, the leading greenhouse gas believed to contribute to global warming;

- $25 \%$ of all emission of nitrogen oxides $\left(\mathrm{NO}_{\mathrm{x}}\right)$, a key component of ozone ( $\mathrm{smog}$ ), acid rain, and fine particulates; and

- $34 \%$ of all emissions of mercury $(\mathrm{Hg})$, a toxic heavy metal that is concentrated through the food chain (EPA 2001).

The source energy saved through the higher efficiency of CHP lowers the amount of emissions that will occur. With 50 TBtu of source energy saved, $\mathrm{CO}_{2}$ emissions would be reduced by $2.7 \mathrm{M}$ metric tons/year, assuming conservatively that all this energy would otherwise have come from natural gas. This is roughly equivalent to the output of 560,000 cars. In some regions of the country, the avoided fuel would be coal or oil for a portion of the energy. These have higher carbon intensities and additional harmful emissions, so pollution prevention benefits of CHP would be much higher. The actual emission benefits of a project will depend on several site-specific and technology specific factors. Using state of the art gas turbines and control technologies, CHP can meet stringent emissions requirements as a clean energy alternative. 
Fig. 11: Federal CHP potential capacity under base case, MW.

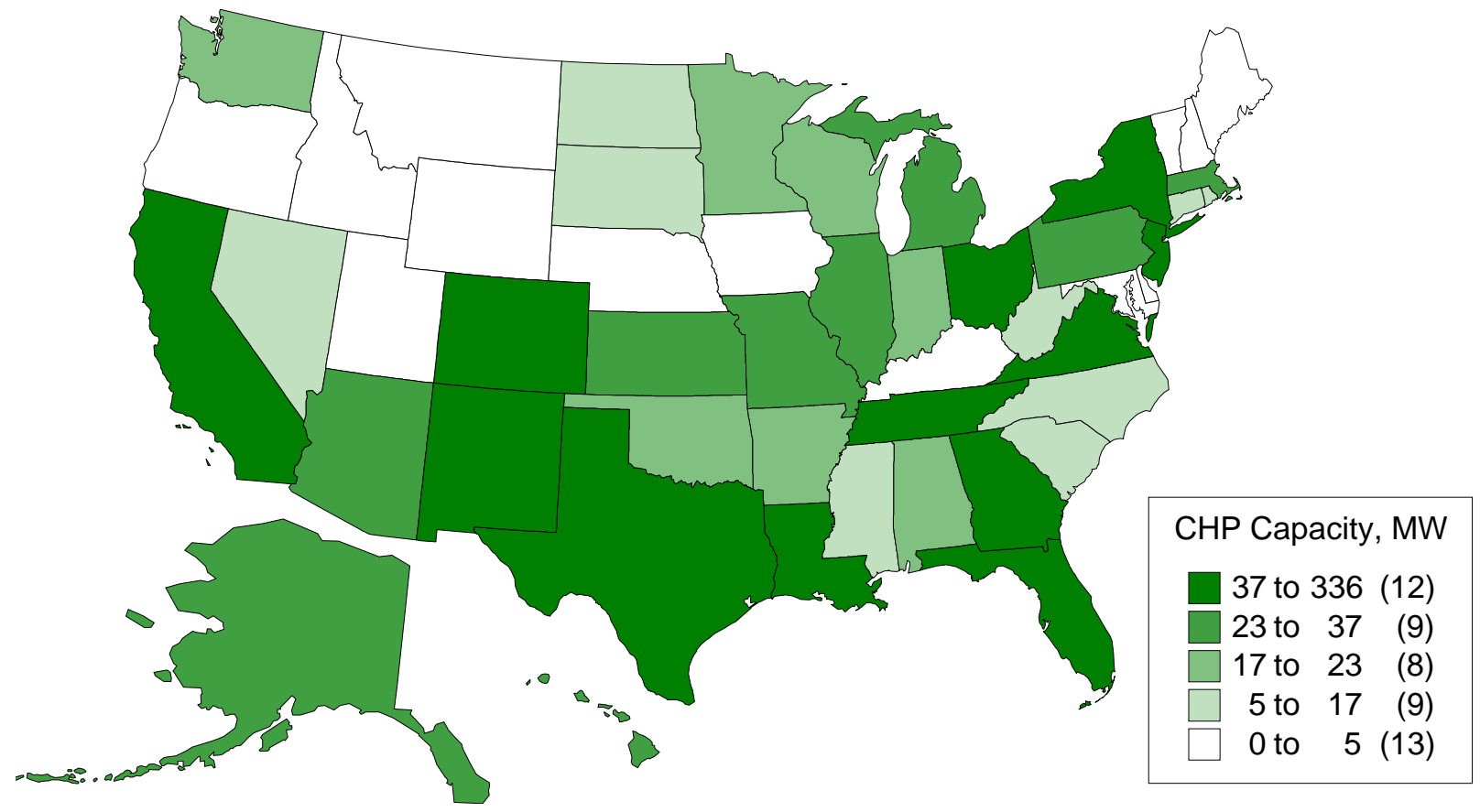

Fig. 12: "Spark spread" difference in electric and gas prices in \$/MBtu.

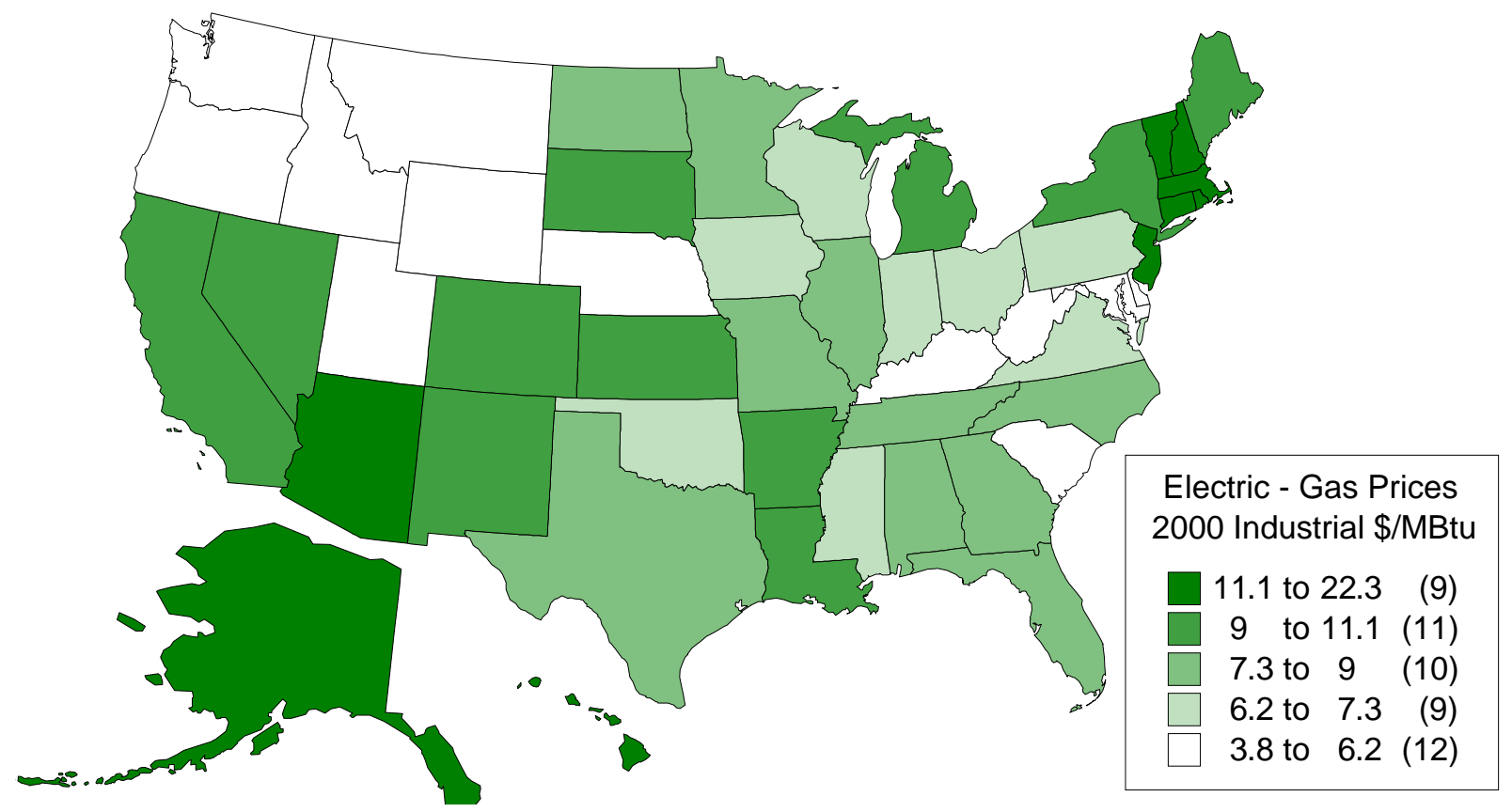




\subsection{Federal CHP Potential by Agency}

The analysis of potential CHP capacity by state did not distinguish among the agencies that own the facilities. The GSA database provides information on the agency and bureau that owns each building. By running the model using each of the 28 agencies' data, we can calculate the potential capacity for each agency in each state. Table 15 shows the total capacity by building type for each agency. Many agencies have little or no potential as calculated using the base case parameters. (The sum does not match the earlier analysis, because agency-by-agency averages by state have slightly different paybacks compared to the building category averages that go above or below the threshold ten years.)

Table 15: Potential CHP capacity by federal agency and building category, MW

\begin{tabular}{l|rrrrrrrr} 
Agency & Hospital Industry & Office & Prison & R\&D & School & Service & Total \\
\hline Air Force & 43 & 57 & 31 & 0 & 85 & 7 & 116 & 339 \\
Veterans Affairs (VA) & 311 & 0 & 1 & 0 & 1 & 0 & 0 & 314 \\
Army & 55 & 101 & 52 & 2 & 24 & 3 & 33 & 270 \\
Navy & 27 & 36 & 39 & 0 & 43 & 2 & 58 & 205 \\
Department of Energy & 0 & 113 & 15 & 0 & 64 & 0 & 2 & 195 \\
National Aeronautics and Space & 0 & 17 & 10 & 0 & 43 & 0 & 3 & 73 \\
Administration (NASA) & 0 & 1 & 68 & 0 & 0 & 0 & 0 & 69 \\
General Services Administration & 0 & 0 & 48 & 0 & 0 & 0 & 0 & 48 \\
United States Postal Service & 0 & 3 & 0 & 34 & 0 & 0 & 0 & 37 \\
Justice & 6 & 0 & 0 & 0 & 2 & 0 & 0 & 9 \\
Health and Human Services & 0 & 8 & 0 & 0 & 0 & 0 & 0 & 8 \\
Treasury & 0 & 0 & 2 & 0 & 4 & 0 & 0 & 7 \\
Transportation & 0 & 2 & 2 & 0 & 0 & 3 & 0 & 7 \\
Interior & 0 & 0 & 0 & 0 & 3 & 0 & 0 & 3 \\
Agriculture & 0 & 0 & 0 & 0 & 2 & 0 & 0 & 2 \\
Commerce & 0 & 0 & 1 & 0 & 1 & 0 & 0 & 2 \\
Corps of Engineers & 0 & 0 & 0 & 0 & 1 & 0 & 0 & 1 \\
National Science Foundation & 0 & 0 & 0 & 0 & 1 & 0 & 0 & 1 \\
Environmental Protection Agency & 0 & 0 & 0 & 0 & 0 & 1 & 0 & 1 \\
Education & 443 & 338 & 269 & 36 & 274 & 16 & 212 & 1588 \\
Grand total & 0 &
\end{tabular}

Note: Other agencies were considered, but did not show potential. These include Agency for International Development, Federal Communications Commission, Federal Emergency Management Agency, Government Printing Office, National Archives and Records Administration, Smithsonian, Department of State, Tennessee Valley Authority.

Nearly all CHP potential is found among nine agencies: the three military services, VA hospitals, DOE, NASA, GSA, the U.S. Postal Service, and the Department of Justice (Fig. 13). And the first three (military, VA and DOE) represent $83 \%$ of the total CHP potential identified in the base case. The military services (over $50 \%$ of total) have significant potential CHP capacity in all types of buildings (except prisons), but the VA has capacity mainly in hospitals (as expected). Energy and NASA capacity is concentrated in R\&D and industrial buildings, while GSA and the Postal Service have capacity in the "office" category. It should be mentioned that the categories directly reflect the GSA database that appears to include Postal Service processing and distribution centers under the office category. The Justice sector capacity is in prisons. 
Fig. 13: Potential CHP capacity for major federal agencies (\% of $1588 \mathrm{MW}$ total).

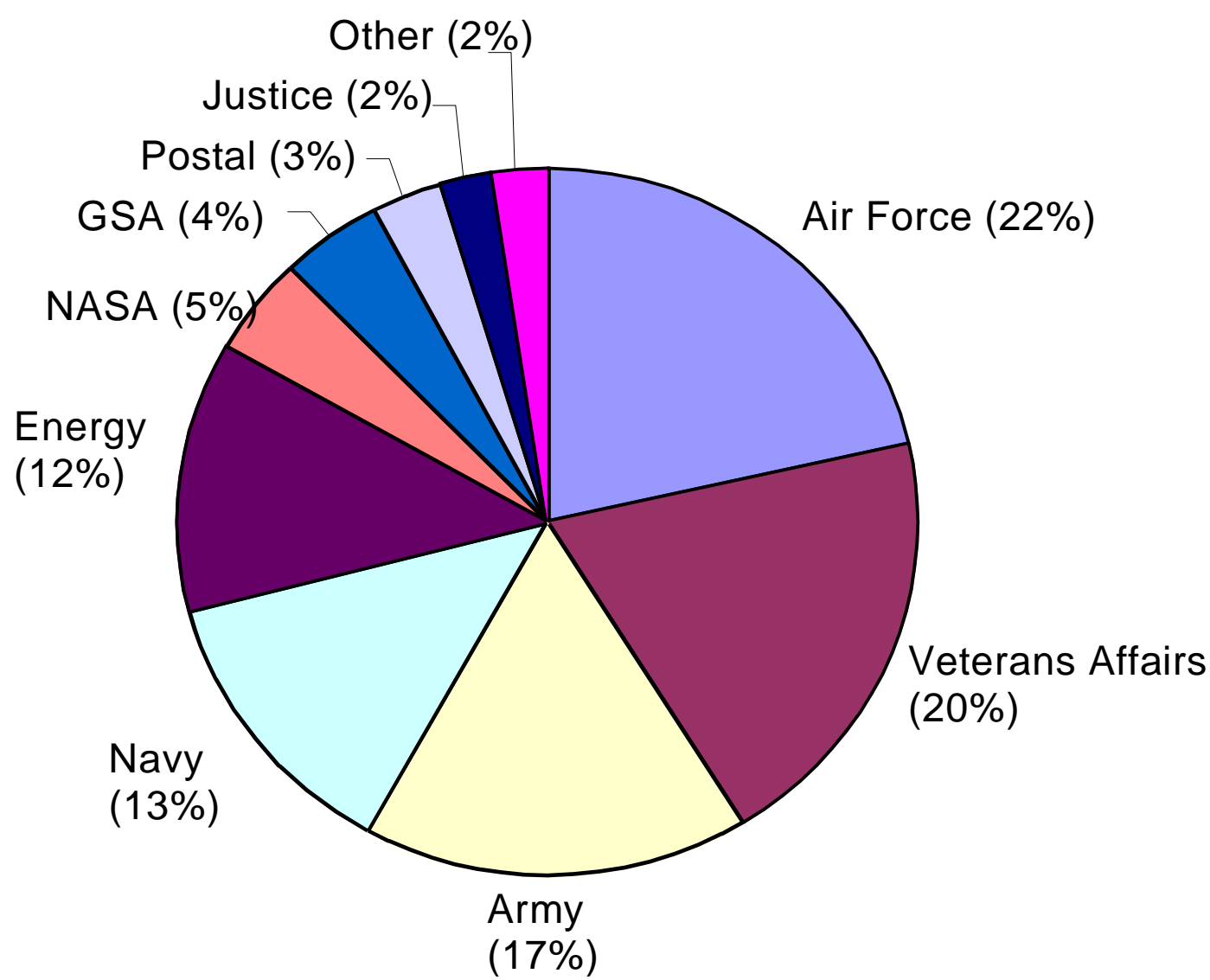

Showing a breakdown of the agency capacities by states gives an idea of where the major agencies have their potential (Table 16). Each agency has its main potential capacity in the states with large facilities and good spark spreads. All show large amounts in California. DOE capacities are in those states with large national laboratories or industrial plants registered in the GSA database. VA hospitals are fairly evenly scattered across the country. While the database is imperfect, the margin of error occurs in both directions: some facilities may close while others are expanding. 
Table 16: Potential CHP capacity by state for leading agencies (MW)

\begin{tabular}{|c|c|c|c|c|c|c|c|c|c|}
\hline State & Air Force & VA & Army & Navy & Energy & NASA & GSA & $\begin{array}{l}\text { Postal } \\
\text { Service }\end{array}$ & Justice \\
\hline AK & 13 & 0 & 6 & 1 & 0 & 0 & 3 & $\overline{1}$ & 0 \\
\hline$A L$ & 1 & 12 & 4 & 0 & 0 & 1 & 0 & 0 & 0 \\
\hline AR & 3 & 8 & 2 & 0 & 0 & 0 & 0 & 0 & 1 \\
\hline$A Z$ & 10 & 4 & 9 & 1 & 0 & 0 & 1 & 1 & 1 \\
\hline CA & 75 & 27 & 13 & 99 & 29 & 25 & 32 & 26 & 5 \\
\hline $\mathrm{CO}$ & 19 & 4 & 14 & 0 & 9 & 0 & 12 & 4 & 2 \\
\hline CT & 0 & 2 & 3 & 4 & 0 & 0 & 1 & 1 & 0 \\
\hline DC & 0 & 5 & 11 & 0 & 0 & 0 & 0 & 0 & 0 \\
\hline $\mathrm{DE}$ & 0 & 0 & 0 & 0 & 0 & 0 & 0 & 0 & 0 \\
\hline $\mathrm{FL}$ & 35 & 14 & 0 & 17 & 0 & 27 & 0 & 0 & 4 \\
\hline GA & 32 & 10 & 6 & 0 & 0 & 0 & 0 & 0 & 4 \\
\hline $\mathrm{HI}$ & 4 & 0 & 10 & 13 & 0 & 0 & 1 & 1 & 0 \\
\hline IA & 0 & 4 & 0 & 0 & 0 & 0 & 0 & 0 & 0 \\
\hline ID & 0 & 0 & 0 & 0 & 0 & 0 & 0 & 0 & 0 \\
\hline IL & 2 & 15 & 8 & 2 & 1 & 0 & 0 & 0 & 2 \\
\hline IN & 0 & 7 & 7 & 4 & 0 & 0 & 0 & 0 & 1 \\
\hline KS & 6 & 4 & 16 & 0 & 0 & 0 & 0 & 0 & 1 \\
\hline $\mathrm{KY}$ & 0 & 0 & 0 & 0 & 0 & 0 & 0 & 0 & 0 \\
\hline LA & 5 & 6 & 10 & 3 & 0 & 10 & 5 & 2 & 1 \\
\hline MA & 8 & 8 & 4 & 2 & 0 & 0 & 4 & 3 & 1 \\
\hline MD & 0 & 0 & 0 & 0 & 0 & 0 & 0 & 0 & 0 \\
\hline ME & 0 & 1 & 0 & 0 & 0 & 0 & 0 & 0 & 0 \\
\hline MI & 2 & 9 & 7 & 0 & 0 & 0 & 3 & 4 & 0 \\
\hline MN & 2 & 6 & 6 & 5 & 0 & 0 & 0 & 0 & 0 \\
\hline $\mathrm{MO}$ & 10 & 7 & 9 & 0 & 7 & 0 & 0 & 0 & 0 \\
\hline MS & 3 & 5 & 2 & 0 & 0 & 0 & 0 & 0 & 0 \\
\hline $\mathrm{MT}$ & 0 & 0 & 0 & 0 & 0 & 0 & 0 & 0 & 0 \\
\hline NC & 0 & 9 & 4 & 3 & 0 & 0 & 0 & 0 & 1 \\
\hline ND & 11 & 1 & 0 & 0 & 0 & 0 & 0 & 0 & 0 \\
\hline $\mathrm{NE}$ & 0 & 0 & 0 & 0 & 0 & 0 & 0 & 0 & 0 \\
\hline $\mathrm{NH}$ & 1 & 1 & 1 & 1 & 0 & 0 & 0 & 0 & 0 \\
\hline $\mathrm{NJ}$ & 10 & 4 & 17 & 5 & 1 & 0 & 3 & 5 & 0 \\
\hline NM & 17 & 2 & 10 & 0 & 37 & 1 & 3 & 1 & 0 \\
\hline NV & 4 & 1 & 1 & 0 & 4 & 0 & 0 & 0 & 0 \\
\hline NY & 6 & 27 & 4 & 5 & 10 & 0 & 0 & 0 & 1 \\
\hline $\mathrm{OH}$ & 3 & 9 & 7 & 0 & 34 & 0 & 0 & 0 & 0 \\
\hline OK & 8 & 4 & 4 & 0 & 0 & 0 & 0 & 0 & 1 \\
\hline OR & 0 & 5 & 0 & 0 & 0 & 0 & 0 & 0 & 0 \\
\hline PA & 0 & 16 & 2 & 1 & 0 & 0 & 0 & 0 & 2 \\
\hline RI & 0 & 1 & 0 & 7 & 0 & 0 & 0 & 0 & 0 \\
\hline SC & 0 & 0 & 0 & 0 & 13 & 0 & 0 & 0 & 0 \\
\hline SD & 8 & 2 & 0 & 0 & 0 & 0 & 0 & 0 & 0 \\
\hline TN & 0 & 14 & 7 & 3 & 38 & 0 & 0 & 0 & 0 \\
\hline TX & 38 & 22 & 40 & 19 & 4 & 10 & 0 & 0 & 8 \\
\hline UT & 0 & 3 & 0 & 0 & 0 & 0 & 0 & 0 & 0 \\
\hline VA & 0 & 10 & 13 & 10 & 0 & 0 & 0 & 0 & 0 \\
\hline VT & 0 & 1 & 0 & 0 & 0 & 0 & 0 & 0 & 0 \\
\hline WA & 0 & 6 & 5 & 0 & 7 & 0 & 0 & 0 & 0 \\
\hline WI & 0 & 9 & 8 & 0 & 0 & 0 & 0 & 0 & 0 \\
\hline WV & 0 & 8 & 0 & 0 & 0 & 0 & 0 & 0 & 0 \\
\hline WY & 0 & 0 & 0 & 0 & 0 & 0 & 0 & 0 & 0 \\
\hline Total & 339 & 314 & 270 & 205 & 195 & 73 & 69 & 48 & 37 \\
\hline
\end{tabular}




\section{Sensitivity Analysis}

While our base case shows 1.5-1.6 GW of potential CHP capacity, it is based on certain assumptions about technology, costs, operating parameters, and building characteristics. If we modify these parameters, the amount of potential CHP capacity (defined as systems with less than ten-year simple payback) changes. Table 17 shows the amount of capacity under the base case and with changes to some of the key parameters.

Table 17: Sensitivity analysis on key CHP parameters

\begin{tabular}{|c|c|c|c|c|c|}
\hline Technology & $\begin{array}{c}\text { Cost \& } \\
\text { efficiency }\end{array}$ & Fuel prices & $\begin{array}{c}\text { Sized for gas or } \\
\text { electric }\end{array}$ & $\begin{array}{l}\text { Building } \\
\text { size }\end{array}$ & $\begin{array}{c}\text { Potential } \\
\text { CHP } \\
\text { MW } \\
\end{array}$ \\
\hline \multicolumn{6}{|c|}{ Technology Cost } \\
\hline Recip. engine & $\begin{array}{l}\text { Current } \\
\text { Future }\end{array}$ & 2000 Industrial & $85 \%$ or $50 \%$ electric & $>25,000 \mathrm{ft}^{2}$ & $\begin{array}{l}1570 \\
2040\end{array}$ \\
\hline “ & $\begin{array}{l}\text { Double current } \\
\text { installed cost }\end{array}$ & “ & “ & “ & 390 \\
\hline \multicolumn{6}{|c|}{ CHP Sizing } \\
\hline Recip. engine & $\begin{array}{l}\text { Current } \\
\text { Future }\end{array}$ & 2000 Industrial & $100 \%$ of gas & " & $\begin{array}{l}1760 \\
2690\end{array}$ \\
\hline “ & Current & “ & $\begin{array}{c}85 \% \text { or } 50 \% \text { electric } \\
\text { w/ credit for max of } \\
100 \% \text { of gas needs } \\
100 \% \text { of gas w/ } \\
\text { credit for max of } \\
100 \% \text { electric }\end{array}$ & “ & 1080 \\
\hline
\end{tabular}

Energy Price

\begin{tabular}{|c|c|c|c|c|c|}
\hline $\begin{array}{l}\text { Recip. engine } \\
\text { “ }\end{array}$ & $\underset{\text { Current }}{\text { Cu }}$ & $\begin{array}{c}2000 \text { Commercial } \\
1999 \text { Industrial } \\
\end{array}$ & $85 \%$ or $50 \%$ electric & " & $\begin{array}{l}2820 \\
2010\end{array}$ \\
\hline \multicolumn{6}{|c|}{ Technology Type } \\
\hline $\begin{array}{l}\text { Turbine } \\
\text { " } \\
\text { Fuel cell } \\
\text { “ }\end{array}$ & $\begin{array}{l}\text { Current } \\
\text { Future } \\
\text { Current } \\
\text { Future } \\
\end{array}$ & $\begin{array}{c}2000 \text { Industrial } \\
\text { “ } \\
\text { “ } \\
\end{array}$ & $\begin{array}{c}85 \% \text { or } 50 \% \text { electric } \\
\text { " } \\
\text { " } \\
\end{array}$ & $\begin{array}{l}" \\
" 1 \\
" \\
\end{array}$ & $\begin{array}{c}1670 \\
2370 \\
0 \\
90 \\
\end{array}$ \\
\hline \multicolumn{6}{|c|}{ Building Size } \\
\hline $\begin{array}{l}\text { Recip. engine } \\
\text { Turbine }\end{array}$ & $\begin{array}{c}\text { Current } \\
\text { “ }\end{array}$ & 2000 Industrial & $85 \%$ or $50 \%$ electric & $\begin{array}{c}25 \mathrm{~K}<\mathrm{x}<100 \mathrm{~K} \\
\|\end{array}$ & $\begin{array}{c}10 \\
9 \\
\end{array}$ \\
\hline
\end{tabular}

The first row shows the base case described above. The second row shows the results if we used the costs and efficiencies that one recent source (RDC 2001) estimates will be available post-2005. The next row shows the effect if the installation cost is twice that reported by RDC. ORNL's experience with FEMP projects have shown a higher installation cost than in Table 8 , although those costs have included additional equipment such as chillers and increased piping. 
Fig. 14: CHP potential capacity (MW) under varying cost and efficiency assumptions.

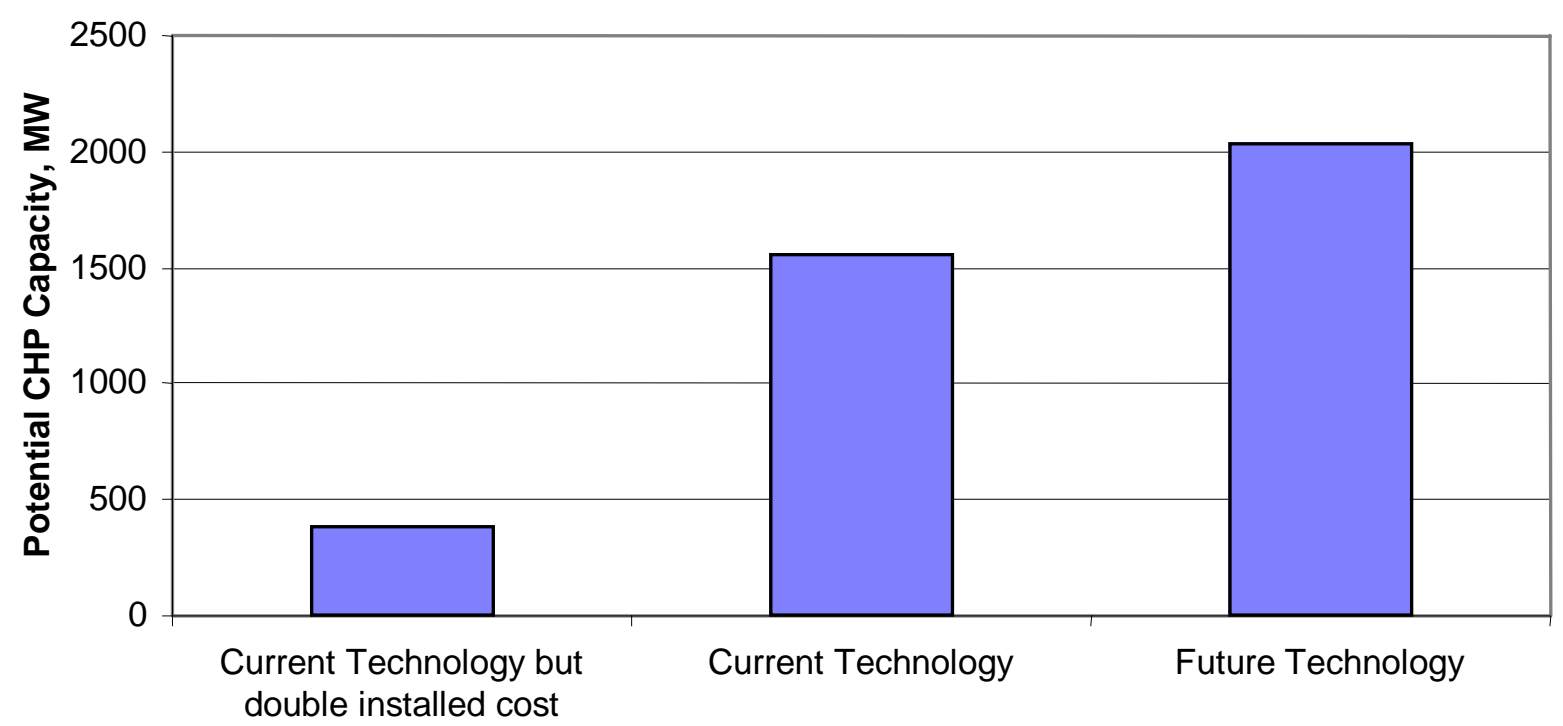

The amount of CHP is very sensitive to the cost and efficiency assumptions. Figure 14 indicates these graphically; there is some increased potential through future improvements that lower installation costs and increase efficiency, but the potential is significantly reduced if installation costs are double those reported by RDC.

Actual projects will have highly site-specific characteristics that influence the costs and potential benefits of a CHP system. While in some cases costs will be higher, in others they could be significantly lower. For example, the base case assumes that the site already has a heating system in good repair. If the site is going to have to replace its heating and cooling system anyway, the installation cost for CHP would be the incremental cost above installing a standard system. This may greatly improve the economics because the savings in power costs would only need to pay back this incremental cost.

The next set of sensitivity analyses varied the amount of energy that the CHP is sized for or receives credit for. The first two assume the same parameters as the base case, but with CHP sized to meet $100 \%$ of current gas needs (according to the CBECS energy intensity data) rather than the base case portion of the electric needs, as shown in row e of Table 10 . The resulting capacity $(1757 \mathrm{MW})$ is similar to but slightly higher than the base case. The gas intensities we used may be based partially on buildings using mechanical chillers or air conditioners. With CHP, they may convert to gas chillers and thereby have additional thermal load. This could raise the amount of CHP potential even higher. The next scenario has the same gas needs but with the future technology costs. Projected capacity rose to 2687 under this scenario, higher than with future technology meeting base case electric needs, since as equipment becomes more efficient it can generate additional electricity for the same amount of thermal exhaust.

Because our base case sized equipment solely on the electric needs of the building, the CHP units may be providing thermal energy in excess of what the buildings can use. If this thermal energy cannot by used in other processes or by neighboring buildings at the site, it will be lost. The next sensitivity sizes the CHP to provide the base case amount of electricity ( $85 \%$ or $50 \%$ depending on building type) but only gives financial credit for thermal exhaust up to $100 \%$ of the building's gas intensity energy needs. Any thermal energy over that is assumed lost. As a consequence, the potential capacity with payback less than 10 years drops significantly to $962 \mathrm{MW}$. Offices have the largest drop, going from $248 \mathrm{MW}$ to $49 \mathrm{MW}$. Their thermal needs are modest in comparison to electrical needs, so if the excess thermal energy has no value, 
then fewer offices have cost-effective potential for CHP. This illustrates the importance of assuring maximum use of recovered heat in CHP applications, both for efficiency and for economic reasons.

Similarly, if we size the equipment based on $100 \%$ of the estimated thermal needs but only give credit for up to $100 \%$ of estimated electric needs for the facility, then potential capacity drops to $1078 \mathrm{MW}$. This sensitivity reflects the assumption that excess electricity cannot be sold to other buildings. In this case, hospitals and service facilities reduce their capacities the most, going from 595 and $323 \mathrm{MW}$ to 274 and $87 \mathrm{MW}$ respectively, because their thermal needs are much higher than their electrical needs, so the loss of electrical credit for excess generation makes more of their capacity uneconomic.

The next two sensitivity analyses look at the prices of gas and electricity, adjusting them to either commercial rates in 2000 or industrial rates in 1999. In both cases, CHP potential is higher than the base case, rising to 2800 and $2000 \mathrm{MW}$ respectively, because the price differential between electricity and gas are higher. The year 2000 saw large increases in gas prices, with non-weighted average prices going from $\$ 3.75$ to $\$ 4.95 / \mathrm{MBtu}(27 \%)$. Industrial electric prices barely changed, going from $\$ 14.01$ to $\$ 14.14 / \mathrm{Mbtu}$. The difference (spark spread) was higher in 1999. Similarly, commercial prices in 2000 for gas and electric had a \$14.05/Mbtu difference between gas and electric, while industrial prices had \$9.19/Mbtu difference. Higher spark spread improves the economics of CHP.

Comparing technology options for the prime mover in a CHP system, current gas combustion turbines had costs and efficiencies similar to reciprocating engines; capital costs were higher but operating costs lower. Electrical efficiency was lower for turbines but since exhaust heat is used by the building rather than wasted, this factor is less important than for an electricity-only distributed generation project. Total capacity for turbines was slightly higher when compared to the base case with engines (Fig. 15). More improvement is expected with turbines than with engines. Using future expected cost and efficiencies, the potential capacity using turbines increased by $700 \mathrm{MW}$, while improvements in engines raised capacity by roughly $500 \mathrm{MW}$.

Fig. 15: Potential CHP capacity with different technologies and performance parameters.

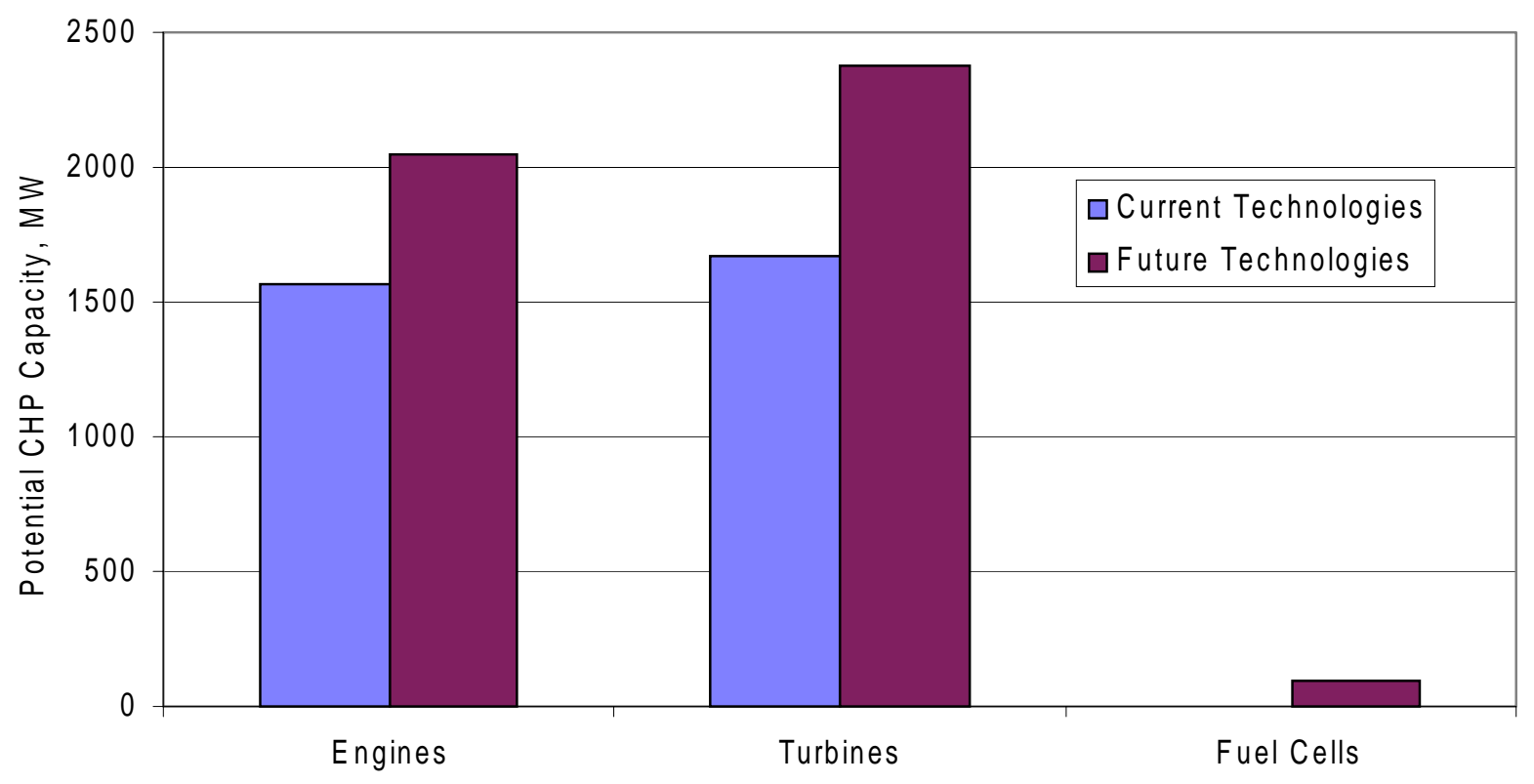


Fuel cells, under current installed costs near $\$ 5000 / \mathrm{kW}$ and $40 \%$ efficiency, had no capacity appear cost effective. Using proposed future installation costs of around $\$ 1500 / \mathrm{kW}$ and $50 \%$ efficiency, $90 \mathrm{MW}$ of capacity falls within the ten-year payback, but no fuel stack replacement is assumed in the O\&M costs. Further improvements in fuel cell costs will be needed for them to be able to penetrate the market.

Looking at the smaller buildings, there was very little CHP potential. At smaller sizes, the reciprocating engines are slightly more cost effective than the turbines. In smaller buildings (between 25,000 and $100,000 \mathrm{ft}^{2}$ ) there were $10 \mathrm{MW}$ of engines that met the economic criteria, but only $9 \mathrm{MW}$ of turbines.

Our base case used all projects with paybacks less than ten years. Some have paybacks significantly shorter than ten years, while there is additional capacity available if we consider longer-term paybacks. Figure 16 shows the amount of capacity in the base case that fell within different payback periods. Beyond the $1567 \mathrm{MW}$ with paybacks less than ten years, there were more than $745 \mathrm{MW}$ with simple paybacks between 10 and 15 years. Under some situations these projects could prove to be economic. However, the "time value" of money makes these less attractive (Fig. 8).

Fig. 16: CHP capacity in base case at different ranges of payback period.

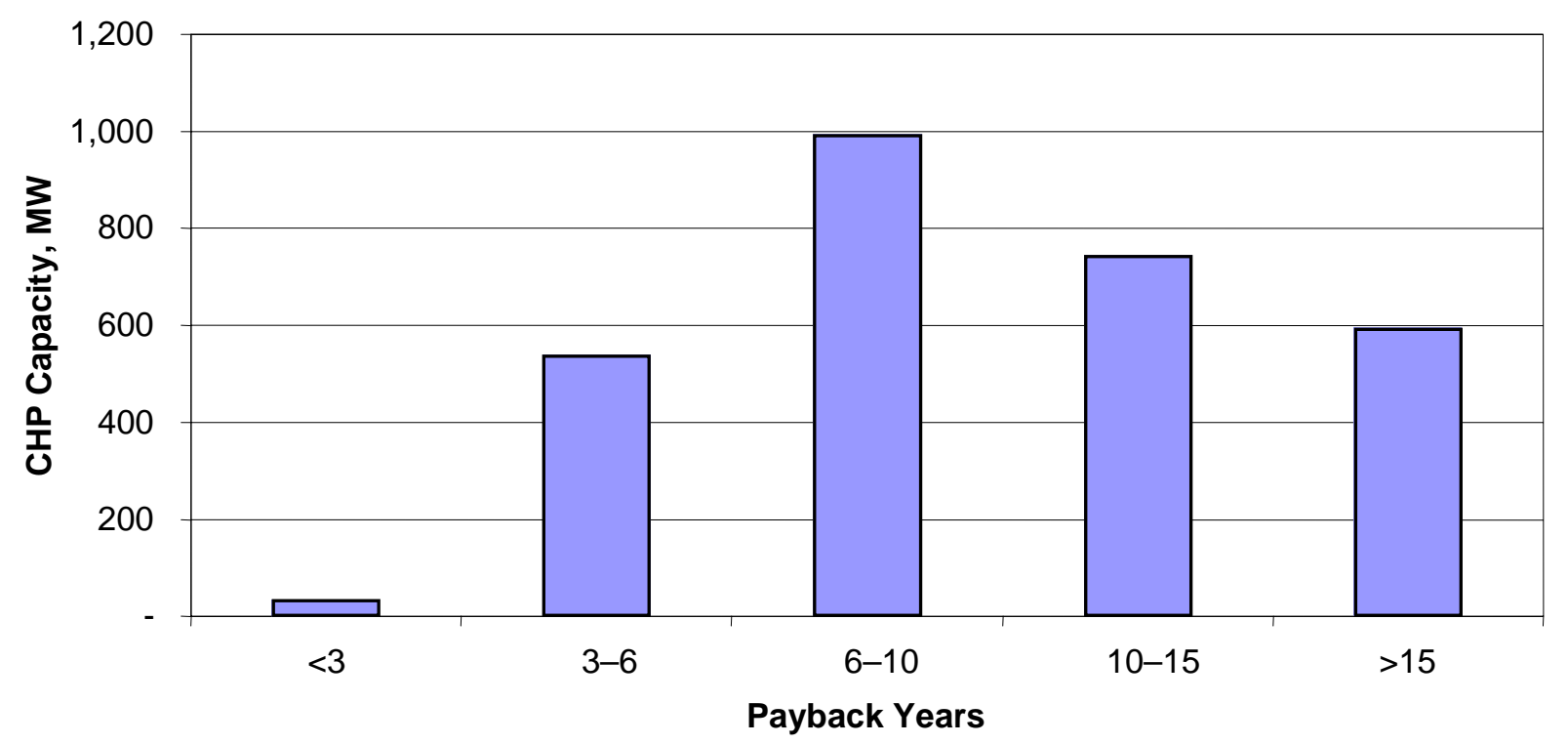




\section{Conclusions}

\subsection{Data Limitations and Further Studies}

Given the quality and types of data available and the methodology used in this analysis, it is impossible to make reliable predictions about specific sites and their respective CHP potentials. This analysis used state- or region-wide averages for various building types and energy variables; any actual site can have widely different values. In addition, so many site-specific conditions affect the economics of a CHP application that it is extremely difficult to make accurate predictions without more detailed, site-specific data. For example:

- The GSA facilities data provide square footage by building type but fail to identify those facilities served by district energy systems. District systems are significant enhancers to CHP economics since they allow for larger equipment and the aggregation of thermal and electrical needs for many smaller buildings. Most district systems are on military bases, so the data used may underestimate CHP capacity in that sector.

- The analysis assumes there is potential CHP capacity only if it can be shown to pay from savings within a given period of time and under a limited set of assumptions. Other factors will often determine whether a CHP system is installed. Energy security and other mission-critical factors may often be the overriding criteria in the decision to install a CHP system, and in some cases, emissions factors carry significant weight.

- Installation costs of recent CHP projects at federal sites have varied from less than $50 \%$ to $150 \%$ of the equipment costs. Some states and utilities may offer subsidies or reduced tariffs for CHP projects. The interconnection and standby fees can vary considerably from one utility district to another. (See Appendix $\mathrm{C}$ for a discussion of interconnection requirements, standby, and exit fees.) These cost factors can significantly affect project economics.

- The condition and type of current HVAC equipment may facilitate or prevent CHP from being deployed. Retrofitting in office buildings may be more costly than assumed here. On the other hand, if a site needs to replace or renovate boilers and HVAC equipment, the marginal cost of adding CHP may be small and the returns may be higher than assumed.

- The GSA database used does not necessarily reflect updated information on facilities and their use. We sponsored a survey of potential sites in California, Texas, and New York using the GSA list as a starting point. Many military facilities on the GSA list had either closed or significantly changed mission. Also, recent expansion by the Bureau of Prisons does not appear to be fully captured.

- The assumptions based on CBECS about the percentage of buildings with CHP-compatible infrastructure may be conservative for some categories (prisons in particular), and there is clearly the possibility that a CHP system could be cost-effective at sites that were assumed to not possess the prerequisite conditions (gas service and central heat or cooling systems).

- The energy intensities used may not accurately represent the actual building intensities. Federal buildings may be more or less energy intensive than regional averages for CBECS building types. Further, many of the buildings profiled in the survey likely used electricity for mechanical chillers, increasing the electrical intensity but lowering the gas intensity. CHP systems can use the thermal 
exhaust in absorption chillers for air conditioning, thereby altering the electric and thermal energy intensities of the buildings.

- And perhaps most important, specific sites will often have far different prices for electricity or gas than the state average used in this analysis. Those tariffs may be lower in some cases today, but are likely to rise as contracts expire and are renewed in the next few years. Also, this analysis did not attempt to look at the potential for CHP systems to be used in conjunction with peak shaving and load-reduction incentive programs that an increasing number of utilities are offering.

However, despite the many limitations, our analysis does provide reasonable approximations of state or national totals, and can easily show the impact of changing various parameters. Future effort needs to analyze site-specific information and should focus on the building sectors, agencies, and geographic regions with the highest potential.

The model developed for this report estimates the magnitude of CHP that could be implemented under various performance and economic assumptions associated with different applications. This model may be useful for other energy technologies. It can be adapted to estimate the market potential in federal buildings for any energy system based on the cost and performance parameters that a user desires to assess. The model already incorporates a standard set of parameters based on available data for federal buildings including total building space, building type, energy use intensity, fuel costs, and the performance of many prime movers commonly used in CHP applications. These and other variables can be adjusted to meet user needs or updated in the future as new data become available.

\subsection{CHP Potential and FEMP}

There is significant potential-1000 to 2000 MW of capacity - for CHP at federal facilities today. Regions with the greatest CHP potential are the Southwest (CA to TX), northeastern metropolitan areas (NY to DC), and the southeast (FL, GA, AL). Agencies with the most potential are the military, VA, and DOE, especially in hospital, industrial, and R\&D facilities. As energy prices increase and CHP system costs decrease, the amount of cost-effective CHP potential will rise. The actual potential could be higher or lower depending on the specific conditions of any given site.

The $1.5 \mathrm{GW}$ identified under the base case scenario would be sufficient to power more than a million homes and save the federal government $\$ 170 \mathrm{M}$ per year in energy costs. To install the $1.5 \mathrm{GW}$ of electrical CHP generating capacity (all cases where the simple payback period is under ten years) would require an estimated $\$ 1.5-\$ 2$ billion in capital investments. Since the average simple payback period for these projects was 6.2 years, most could be financed through existing credit mechanisms supported by FEMP (ESPC, UESC, etc.). The net primary energy savings from this level of CHP investment are estimated to be 50 trillion Btus per year, the energy equivalent of over $8 \mathrm{M}$ barrels of oil per year. And projected carbon dioxide emissions would be reduced by $2.7 \mathrm{M}$ metric tons per year compared to gas-fired central electric power and thermal alternatives.

There has been a recent upsurge of interest in fuel-efficient distributed energy resources such as CHP among project developers, federal facility managers, and policy makers because these systems can offer significant benefits in terms of dollar savings, emissions reductions, and increased energy security. They also help mitigate other power constraints; meet increased energy demand; reduce transmission congestion; increase power quality and reliability; and in sufficient numbers, interconnected CHP systems can offer increased power security for the grid as well (Casten and Casten 2001). 
CHP in buildings can also help facilitate a transition to cleaner fuels of the future (such as hydrogen) that would rely upon the same infrastructure as CHP and effectively utilize fuel cells when proven, commercial products that are economically feasible to apply become available. EPA considers CHP to be "a proven pollution prevention technology" (EPA 2001a). Over 50,000 MW of CHP capacity was in place in the United States in 2001, primarily in the industrial sector. DOE and EPA would like to see current CHP capacity doubled by 2010 (USCHPA 2001). State public utility commissions, such as those in Texas and California, are leading the way to clarify local regulations for permitting and interconnection of DER in general and CHP in particular. DOE and EPA are collaborating to address several policy issues such as more equitable treatment of CHP systems when looking at air quality standards. (See Appendix D for a discussion of emissions permitting and siting issues.)

FEMP's mission is to reduce the cost to and environmental impact of the federal government by advancing energy efficiency and water conservation, promoting the use of renewable energy, and improving utility management decisions at federal sites. Federal agency sites are FEMP's customers and FEMP's programs are customer-driven. While FEMP is not a technology development program, it does monitor energy efficiency and renewable energy technology developments and mounts "technologyspecific" programs to make technologies that are in strong demand by agencies easily accessible to them. Sometimes these technologies are the product of R\&D sponsored by sectors within DOE's Office of Energy Efficiency and Renewable Energy (EERE). In those cases, FEMP's role becomes one of helping the federal government "lead by example" through the use of advanced EERE technologies in its own buildings and facilities.

CHP was highlighted in the Bush Administration's National Energy Policy Report as a commercially available technology offering extraordinary benefits in terms of energy efficiencies and emission reductions. FEMP's criteria for emphasizing a technology are that it be commercially available; be proven but underutilized; have a strong constituency and momentum; offer large energy savings and other benefits of interest to federal sites and FEMP mission; be in demand; and carry sufficient federal market potential. CHP meets all of these criteria, with the latter documented in this report and in subsequent sitespecific screening activities.

Although CHP technologies are proven and the potential savings and benefits are significant, project development over the past decade has been modest in the federal sector. Given the potential for CHP, why haven't more federal facilities installed this technology? Preliminary discussions with federal facility managers suggest that the primary reasons include:

- low historical tariffs for electricity;

- high initial cost of CHP systems;

- limited budgets (agencies rarely have sufficient appropriations for even much smaller energy conservation investments);

- complexity of CHP systems due in part to the need for custom engineering and design of different components for each site;

- a lack of time and capability for facility managers to evaluate potential applications and benefits to their site;

- obstacles related to local regulations and policies for interconnection, standby/backup charges, siting and emissions (see Appendixes $\mathrm{C}$ and D); and

- a lack of trusted sources of information about the costs, operation and performance of CHP systems.

FEMP is working to address many of the obstacles through technical and project financing assistance, education and outreach. An initiative called "Accelerated Development and Deployment of Combined Cooling, Heat, and Power," or ADD CHP, is an integral part of FEMP's overall program. The strategy is 
to enable sound investments in CHP systems by providing qualified support to federal sites with champions motivated to develop a CHP project. FEMP services, resources permitting, include:

- conducting CHP quick technical screening for interested federal sites;

- performing site survey and feasibility verification;

- fostering partnerships between federal, state, and private sector project developers and financiers;

- collecting baseline data;

- fostering partnerships between federal sites and industry developers of "packaged" CHP;

- providing design and technical assistance to projects selected under FEMP calls for projects;

- providing support in addressing policy and regulatory constraints - siting and permitting, grid interconnection requirements, exit fees, backup charges;

- providing conceptual design, component matching, and sizing verification (thermal/power profiles); and

- evaluating technical/price proposals

\subsection{How to Determine Whether a Facility Has CHP Potential}

Many federal facility managers have no time to investigate whether CHP will work for their site. FEMP can assist through a free screening for CHP potential. The screening provides an initial estimate of sitespecific economics for a CHP project and helps determine if further investigation of CHP opportunities is worth the effort. Some of the basic criteria that will influence the economics of a CHP project are listed in the sidebar. Several other factors affect the economics of CHP projects, for example, if CHP is linked to replacement of equipment nearing the end of its useful life, or if it is bundled with other energy-efficient measures with shorter payback periods, economics could improve significantly. And as demonstrated

\section{Do you have CHP potential?}

Ideal sites will fit the following profile, but sites meeting only a few of these characteristics may also have a cost-effective CHP opportunity:

$\checkmark$ high electric prices (more than 5 cents $/ \mathrm{kWh}$ );

$\checkmark$ average electric load greater than $1 \mathrm{MW}$;

$\checkmark$ ratio of average electric load to peak load $>0.7$

$\checkmark$ a central or district heating and/or cooling system in place (or a need for process heat)

$\checkmark$ "spark spread" (difference in price per million site Btu between gas and electricity) $>\$ 12$

$\checkmark$ high annual operating hours (>6000)

$\checkmark$ thermal demand closely matches electric load earlier, CHP economics are highly sensitive to utility rates. CHP systems could help a facility "flatten" the peaks in electric and gas loads, allowing sites to negotiate reductions in rates and demand charges or to move to a more favorable interruptible rate schedule for part of the load. On the other hand, there could be significant costs related

to standby and exit fees. Therefore, once an initial screening indicates there is potential for CHP, it is recommended that sites investigate utility rate issues and opportunities that may arise with the CHP project along with siting and permitting issues (Appendixes $\mathrm{C}$ and $\mathrm{D}$ ).

Strong private partners can support the CHP project development process as well as offer a source of financing. And of course FEMP is available to assist federal sites in their efforts to identify appropriate partners and deploy CHP. FEMP recognizes the significant potential for CHP technologies to reduce the costs of government, increase energy security, and improve air quality and is actively working to make advanced CHP technologies more easily accessible to federal agencies throughout the nation. 


\section{References}

Alderfer, R. Brent, M. Eldridge, and T. Starrs, 2000. Making Connections: Case Studies of Interconnection Barriers and their Impacts on Distributed Power Projects. NREL/SR-20028053, May.

Casten, Thomas and S. Casten, 2001. Transforming Electricity. Northeast Midwest Economic Review, Nov/Dec.

DOE 2000, Energy Efficiency Improvements Through the Use of Combined Heat and Power (CHP) in Buildings, DOE/EE-0239; published by ORNL, October, 2000. http://www.eren.doe.gov/femp/prodtech/pdfs/chp tf.pdf.

EIA 1998. Energy Information Administration, A Look at Commercial Buildings in 1995: Characteristics, Energy Consumption, and Energy Expenditures, DOE/EIA-625(95), U.S. Department of Energy, Washington, DC, November. http://www.eia.doe.gov/emeu/cbecs/report_1995.html

EIA 2001. Energy Information Administration, Natural Gas Monthly, DOE/EIA-0130 (2001/08), August. http://www.eia.doe.gov/oil_gas/natural_gas/data_publications/natural_gas_monthly/ngm.html

EPA 2001a. CHP, Combined Heat and Power Partnership, information bulletin (October). CHP Partnership, Mail Code 6202J, 1200 Pennsylvania Ave, NW, Washington, DC.

EPA 2001b. from the introduction to the EPA Emissions and Generation Resource Integrated Data Base, (EGRID). http://www.epa.gov/airmarkets/egrid/

FEMP 1999. Executive Order 13123, "Greening the Government Through Efficient Energy Management." www.eren.doe.gov/femp/aboutfemp/exec13123.html.

FEMP 2000. Annual Report to Congress on Federal Government Energy Management and Conservation Programs Fiscal Year 1998. DOE/EE-0221, March 20. USDOE, EERE. Washington, DC.

FEMP 2001. Annual Report to Congress on Federal Government Energy Management and Conservation Programs Fiscal Year 1999. DOE/EE-0252, May 10. USDOE, EERE. Washington, DC.

FEMP 2002. Annual Report to Congress on Federal Government Energy Management and Conservation Programs Fiscal Year 2000. (Draft) USDOE, EERE. Washington, DC. 2002.

GSA 2001. General Services Administration, GSA Real Property Database, (Carole Anadale: 202-2082970).

GSA 1997. General Services Administration Real Property Reporting Instructions, Federal Property Management Regulations, Amendment A-54. David J. Barram, Administrator of General Services. 
NEPDG 2001. National Energy Policy Development Group 2001, National Energy Policy Report, Reliable, Affordable, and Environmentally Sound Energy for America's Future, Office of the President of the United States, May 2001. http://www.whitehouse.gov/energy/

OnSite Energy Corporation, Energy Nexus Group 2001. Screening of CHP Potential at Federal Sites in Select Regions of the U.S. Prepared for ORNL under DOE contract, December.

ORNL 1994. Analysis of Savings Due to Multiple Energy Retrofits in a Large Office Building, H. McLain, S. Leigh, M. MacDonald, DOE ORNL/CON-363, May.

ORNL 1988. An Analytical Investigation of Energy End-Use in Commercial Office Buildings, H. McLain, M. MacDonald, D. Downing; for GRI and DOE, ORNL CON-250, March.

RDI 2001a. PowerDat Database, Resource Data International, Boulder, CO.

RDC 2000. Resource Dynamics Corporation, Building Cooling, Heating, and Power (BCHP): A Market Assessment, Draft, May.

USCHPA 2001. United States Combined Heat and Power Association, in cooperation with DOE and EPA, National CHP Roadmap, Doubling Combined Heat and Power in the United States by 2010, October. http://www.nemw.org/uschpa or http://www.eren.doe.gov/der/chp. 


\section{Appendix A: GSA Federal Building Data Base Categories Defined}

Source: General Services Administration Real Property --Reporting Instructions

June 9, 1997, Federal Property Management Regulations, Amendment A-54.

Section III - Buildings, Block 36. Usage Code and Classification. The following two-digit code numbers indicate a building's predominant use:

10: Office. Buildings primarily used for office space.

14: Post Office. Buildings or portions of Buildings used as a Post Office.

21: Hospital. Buildings used primarily $\mathrm{f}$ or furnishing in-patient diagnosis and treatment under physician supervision and having 24-h-a-day registered graduate nursing services. This category also includes medical laboratories used for routine testing. This category excludes buildings used directly in basic or applied medical research.

22: Prison (Government-owned only). Buildings under the jurisdiction of the Department of Justice used for the confinement of Federal prisoners.

23: School. Buildings used primarily for formally organized instruction, such as schools for dependent children of Federal employees, Indian schools, and military training buildings.

29: Other Institutional Uses. Buildings used for institutional purposes other than schools, hospitals, and prisons, such as libraries, chapels, museums, and outpatient clinics.

30: Housing. Buildings primarily used as dwellings, such as apartment houses, single houses, row houses, barracks, public housing, military personnel housing, Federal employee housing, and housing for institutional personnel.

40: Storage. Buildings used for storage, such as warehouses, ammunition storage, cover sheds, garages primarily used for storage of vehicles or materials. This category excludes water reservoirs and oil storage tanks.

50: Industrial. Buildings specifically designed and primarily used for production or manufacturing, such as the production or manufacture of ammunition, aircraft, ships, vehicles, electronic equipment, chemicals, aluminum, and magnesium.

60: Service. Buildings used for service activities, such as maintenance and repair shops, dry cleaning plants, post exchange stores, airport hangars, and garages primarily used for vehicle maintenance and repair.

70: Research and Development. Buildings used directly in basic or applied research in the sciences (including medicine) and in engineering, such as medical laboratories; meteorological research laboratories; and buildings used in designing, developing, and testing of prototypes and processes for chemistry and physics. This category excludes medical or industrial laboratories used for routine testing.

80: All Other. Buildings which cannot be classified elsewhere.

99: Trust Buildings. All buildings held in trust by your agency. 
This page intentionally left blank. 


\title{
Appendix B: Methods Used to Perform Statistical Analysis on CBECS 95
}

\author{
CBECS 95 (Commercial Buildings Energy Consumption Survey 1995) was used to estimate \\ quantities/percentages of buildings likely to have HVAC infrastructure compatible with a CHP system. \\ The CBECS is a national sample survey collected by the U.S. Energy Information Administration (EIA). \\ The EIA collects data on energy-related building characteristics and energy consumption and \\ expenditures for commercial buildings in the United States. The CBECS was conducted triennially \\ between 1979 and 1995. CBECS 95 contains 5766 observations that are weighted to approximate all \\ commercial buildings in the U.S. For more information on CBECS, see \\ http://www.eia.doe.gov/emeu/cbecs/contents.html.
}

Files 1, 3, and 4 of CBECS 95 were read into SAS and merged into one data file. (File 1 is the base file, File 3 is the heating equipment file, and File 4 is the cooling equipment file.) CBECS divides principal building activity into the following categories: Vacant, Office/Professional, Mercantile/Services, Laboratory, Warehouse (Nonrefrigerated), Food Sales, Public Order and Safety, Health Care (Outpatient), Industrial Processing/Manufacturing, Agricultural Purposes, Warehouse (Refrigerated), Religious Worship, Public Assembly, Education, Food Services (Restaurants), Health Care (Inpatient), Nursing Home, Lodging (Hotel/Motel/Dorm), Residential, Indoor Parking Garage, Strip Shopping, Enclosed Shopping Center/Mall, Retail (Excluding Mall), Service (Excluding Food), Other, and Don't Know. For purposes of this report, all activities other than Office/Professional, Laboratory, Public Order and Safety, Industrial Processing/Manufacturing, Education, and Health Care (Inpatient) were coded to "Other."

Several logical variables for the new data file were created based on variables in the CBECS data file. Observations were separated according to whether the square footages of the buildings they represented were less than or greater than $100,000 \mathrm{ft}^{2}$.

The variable NGUSED6 indicates if the building used natural gas in 1995. CBECS does not have a variable to identify an unused natural gas supply. Buildings were assumed to have natural gas and a central heating system if they used natural gas in 1995 and if they had either district steam or hot water or a boiler. Buildings were assumed to have natural gas and a central cooling system if they used natural gas and either district chilled water piped in or central chillers inside building.

The variable MAINHT6 lists various types of main heating equipment. If MAINHT6 had values of 5 (district steam or hot water) or 6 (boiler), the building was assumed to have a central heating system (district hot water or steam). In a similar vein, MAINCL6 lists various types of main cooling equipment. If MAINCL6 had values of 5 (district chill water piped in) or 6 (central chillers inside building), the building was assumed to have a central cooling system (district chilled or central chiller).

For other CBECS categories, it was difficult to determine if an existing system would be compatible with CHP. If the variable for "Furnace heat distributed by vents" (FURNDC6) had a value of 1 (Yes), then the building was assumed to have central forced air heating. If the variables for "Residential-type central air conditioner" (RCAC6) or "Central Air Distributed by Vents" (RCACDC6) had a value of 1 (Yes), then the building was assumed to have central forced air cooling. Air conditioning wall/window units, heat pumps, packaged cooling units, evaporative cooling units, and other cooling systems were assumed to be incompatible with CHP for this analysis.

Buildings were assumed to have natural gas and either central heating or cooling or central forced air if they used natural gas and any of the following: district steam or hot water, a boiler, district chill water piped in, central chillers inside building, or central forced air heating or cooling. Buildings were assumed to have natural gas and both central heating and cooling if they used natural gas, and both MAINHT6 and 
MAINCL6 had values of either 5 or 6. "If-Then" statements based on these assumptions were used to create logical variables that represented each of these building conditions.

Each observation in CBECS is weighted by EIA, based on how many similar U.S. buildings the specific observation represents. Using the weighting, a frequency procedure was run in SAS. The frequency procedure produced tables that showed weighted frequency of occurrence and percentage of the selected building conditions versus the building types. The frequency procedure was run for all U.S. buildings, buildings less than 100,000 $\mathrm{ft}^{2}$, and buildings greater than $100,000 \mathrm{ft}^{2}$. Although the database contains only 165 buildings owned and operated by the federal government, these buildings were separated from the database through creation of a logical variable. The same frequency procedure was run for all federal buildings and for buildings less than and greater than $100,000 \mathrm{ft}^{2}$. The results of these frequency procedures are shown in the tables in the text of this report. 


\section{Appendix C: Utility Interconnection Requirements, Exit Fees, Stand-By Fees}

Source: Energy Nexus Group, Screening of CHP Potential at Federal Sites in Select Regions of the U.S. Prepared for ORNL under DOE contract; December 2001.

\section{Grid Interconnection}

Non-standard, out-dated, and overly stringent grid interconnection requirements have been a barrier to widespread deployment of distributed generation technologies. Interconnect requirements vary by state and/or utility and are often not based on state-of-the-art technology or data. Compliance often requires custom engineering and lengthy negotiations that add cost and time to system installation. These requirements can be especially burdensome to smaller systems (under $500 \mathrm{~kW}$ ). Non-standard requirements also make it difficult for equipment manufacturers to design and produce modular packages. Lack of interstate or intrastate uniformity discourages the economic business case for CHP in all markets and applications.

Utilities have many legitimate interconnection concerns. First, safety of line personnel must be maintained at all times. This means the CHP system must provide assurance that in the event the utility takes a line out of service for maintenance or any other reason, the CHP system will not inadvertently energize this circuit. Second, safety of equipment must not be compromised in any way. A CHP system failure must not cause damage to other customer sites or to the system to which it is connected. Reciprocally, a utility distribution system fault must not have the ability to damage the CHP system. Third, the reliability of the CHP system must not be compromised in any way.

Public utility commissions in California, Texas, and New York are focusing on interconnection issues and are moving toward the development of more equitable standards. The results from these efforts will help define the issues more clearly. Industry standards organizations, especially the Institute of Electrical and Electronic Engineers (IEEE), have provided a forum for developing consensus based national interconnection standards (IEEE Standards Coordinating Committee 21 on Fuel Cells, Photovoltaics, Dispersed Generation and Energy Storage). The Electric Power Research Institute (EPRI) is beginning to evaluate the effect of increased deployment of on-site generation on grid system dynamics and is exploring the development of controls and communications protocols to facilitate grid dispatch and interface.

\section{Exit Fees and Potential Stranded Assets}

Electric utilities have argued that a customer's installation of onsite generation may strand physical assets already existing that were placed into service for that particular customer's use. The utilities' position is that stranded assets can consist of portions of the distribution system, transmission system, or even generating capacity. When a customer withdraws his load from the system, the cost of these stranded assets can place a financial burden on the utility or its other customers. This argument then forms the basis of the utilities' contention that they are allowed to collect exit fees from those customers withdrawing their load from the system. Exit fees can consist of a one-time charge or even a surcharge levied against every kWh that the onsite unit generates. 


\section{Standby/Back-up Charges}

On-site CHP usually requires back-up power for protection in the event that the on-site generation system suffers unexpected outages or to cover routine system maintenance. Currently, utilities charge for power used and for reserved generation and distribution capacity. Unreasonably high charges for these services can be barriers to on-site generation. In a restructured market, the generation back-up charge will be negotiated between the user and generation supplier.

Regulators will continue to set the distribution rates, attempting to balance utility and user needs and to protect the interest of ratepayers. The burden of the standby charges can sometimes be high but many utilities have established fair rates based on the investment cost to hold that capacity available ( $\$ 25-\$ 35 / \mathrm{kW}$-year). Utilities are increasingly offering new rate schedules for on-site power generators that recognize their potential to support demand side management.

Federal facility managers planning CHP should talk to their electric utility and consider these interconnection issues early in the project development phase to avoid surprises later. They should also talk to their gas utility about supply issues and obtaining improved rates if they were to install a CHP plant with an increased and stable load. 


\section{Appendix D: Emissions Permitting and Siting}

Source: Energy Nexus Group, Screening of CHP Potential at Federal Sites in Select Regions of the U.S. Prepared for ORNL under DOE contract; December 2001 (draft).

As with other regulatory issues (standby, exit fees in Appendix C), federal facility managers considering a CHP project should identify emission and siting issues early in the project development phase to avoid surprises later. Projects located in geographic areas that are classified as "non-attainment" (e.g. areas that do not meet or attain clean air standards), or that are located in or near other areas of special environmental concern (national parks) will face special permitting challenges.

Emissions permitting involves approvals and requirements set by state or local air pollution emission control agencies necessary to construct and operate the facility. CHP emissions are related to the type of fuel, power production technology, and emission control technologies. Major CHP facilities today will most often use natural gas in either combustion turbines or reciprocating engines. The key regulated emission from natural gas is $\mathrm{NO}_{\mathrm{x}}$. This combustion product is strictly regulated under the Federal Clean Air Act (CAA) because it is often a precursor of ozone pollution. Figure D-1 indicates ozone "nonattainment" counties in the U.S. as defined nationally by the EPA. Permitting new CHP facilities in the nonattainment areas portrayed in the figure can be a relatively difficult and costly process (although much less so if the project replaces an older system with higher emission levels). Therefore, if other factors are equal, opportunities for new CHP facilities are more attractive in attainment areas than in nonattainment areas. When a new CHP system replaces older equipment, there will normally be a significant

Fig. D-1: Ozone nonattainment counties in the United

UNTED STATES

Nonatlounment Designotions for Ozone as of August 2001
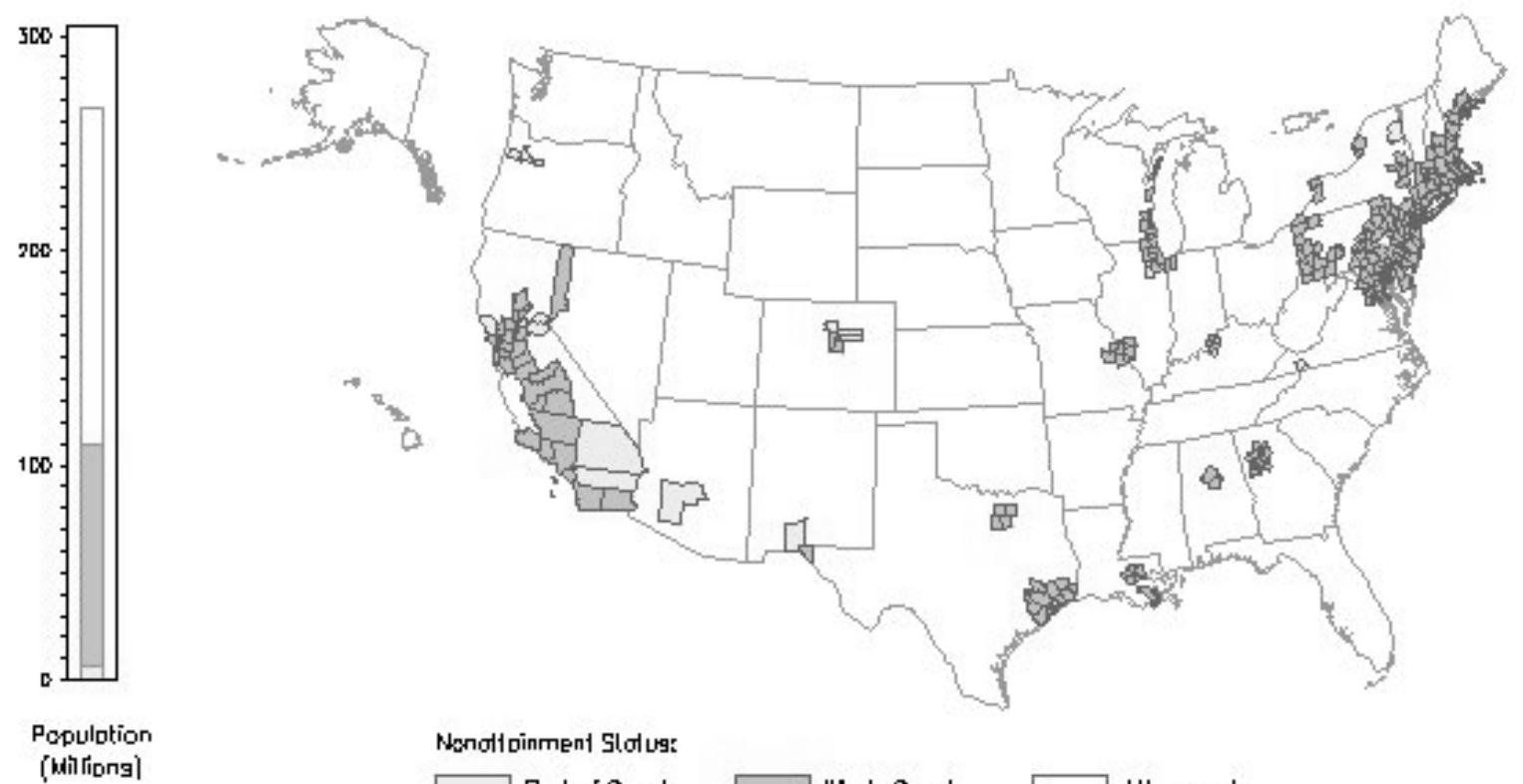

Nonditoinment Slolug:

Port of courly

Whok County A.t1oınmert 
reduction in regulated emissions. In those cases, the difference in emissions can sometimes facilitate and provide significant financial support to the project.

The following URL gives the current status of EPA non-attainment areas for ozone: http://www.epa.gov/oar/oaqps/greenbk/onc.html. And maps of EPA nonattainment areas for various regulated emissions can be found at:

www.eq.state.ut.us/EQAIR/GRAPHICS/MAPS/non_attn.pdf. While these federal non-attainment zone are important, actual CHP permitting and emission requirements are established at the state and local level where additional requirements and nonattainment areas are often established as discussed in more detail below.

Operators in the nonattainment zones are generally required to install the best available control technology (BACT) for emissions reduction. This imposes an additional cost, both up front and in operating costs, as well as a regulatory burden. For gas turbines, uncontrolled $\mathrm{NO}_{\mathrm{x}}$ emissions of $175 \mathrm{ppm}$ can be reduced to $25-42 \mathrm{ppm}$ with steam or water injection, or 10-25 ppm with dry, low $\mathrm{NO}_{\mathrm{x}}$ combustor designs, and 5-10 ppm using selective catalytic reduction (SCR). But there are costs for the equipment, water processing and maintenance of these additional controls. For example, SCRs can add $10 \%$ to the initial equipment costs and increase operating and maintenance costs by $15-20 \%$ due to the need to purchase ammonia and replace catalyst components periodically. Besides the higher costs of the technologies required in nonattainment areas, these areas may require new sources of $\mathrm{NO}_{\mathrm{x}}$ and other criteria pollutants to "offset" their incremental emissions. This may increase project costs because of the necessity to purchase "pollution credits" (allowances), or it may help finance the project through the sale of credits from older, less-efficient systems.

The EPA has recognized the potential for CHP systems to help reduce overall emissions and meet air quality objectives through its high overall efficiency and lower emissions per output of useful energy compared to most alternatives. For these reasons, EPA launched a "Combined Heat and Power Partnership" to expand CHP applications nationwide (EPA 2001a). The EPA partnership has offered support to federal facilities facing permitting issues for new CHP plants. And EPA is working on regulatory reforms that will credit CHP technologies for their higher source energy efficiency because CHP displaces energy and pollution generated from other sources. A great resource for comparing local emissions and mapping air quality issues is available from the EPA "Emissions and Generation Resource Integrated Database, EGRID" at http://www.epa.gov/airmarkets/egrid/.

All new stationary sources emitting criteria pollutants such as $\mathrm{NO}_{\mathrm{x}}$ must go through a New Source Review (NSR) process determined by the state or local air pollution control agency. The general requirement for any new facility is that the pollutant source is equipped with the BACT. When a facility is considered a "major" source, it must also have an operating permit. The required operating permit falls under part 70 of Title $\mathrm{V}$ of the CAA, but the permitting process is determined by the state or local pollution control agency designated to administer it.

Among technologies using natural gas, fuel cells and combustion turbines are particularly attractive in nonattainment areas because they have significantly lower rates of $\mathrm{NO}_{\mathrm{x}}$ emissions than do reciprocating engines. For instance, Table D-1 indicates the expected technologies that will meet regulatory requirements for distributed generation (DG) technologies in Texas. Siting CHP/DG technologies in east Texas is more restrictive than in west Texas because east Texas includes nonattainment areas. 
Table D-1. Example of technologies with potential to meet Texas emission regulations

East Texas

West Texas

\begin{tabular}{l|l}
\hline Fuel cells & All technologies allowed in east Texas plus: \\
\hline Micro turbines & Clean reciprocating engines \\
\hline $\begin{array}{l}\text { Clean turbines using catalytic } \\
\text { combustors or flue gas cleanup }\end{array}$ & $\begin{array}{l}\text { Clean diesel engines operating as peaking } \\
\text { units }\end{array}$ \\
\hline $\begin{array}{l}\text { Cleanest reciprocating engines using } \\
\text { catalytic converters }\end{array}$ & \\
\hline
\end{tabular}

In summary, states and/or local pollution control agencies have significant discretion within the general regulations required by the CAA including setting requirements that may be stricter than EPA guideline requirements. Therefore, although much can be learned from Federal regulations and guidelines, the state or local agency responsible for the State Implementation Plan for the CAA should be considered as the first and final authority for what will be required. The following URL provides links for state and local air pollution control agencies:

http://www.cleanairworld.org/scripts/us_temp.asp?id=307

Another resource for sorting out the permits required for CHP facilities are the many consulting firms that specialize in dealing with the permitting process. Using such consulting firms may be a good investment in planning and carrying out a project. State permitting agencies usually maintain lists of such consulting firms. Federal sites interested in CHP can also consider soliciting proposals for a "turnkey" project in which siting, permitting and emission issues are addressed by the contractor and resolved prior to acceptance.

\section{Other Siting Barriers}

Besides emission permits, siting of CHP equipment involves approval by local agencies and acceptance by the affected communities. Permits may be required from the local fire department, building department, and planning department. On a policy and planning level, local community planning groups may also be involved; such groups monitor the growth issues of their community, as well as actively participate in the land use planning issues.

Many concerns and issues involved in the CHP siting process are legitimate land-use planning issues. The additional burden on CHP comes from a lack of knowledge by local authorities and community leaders of CHP technologies. This is especially true for newer technologies and small CHP equipment applications. Most CHP equipment operations are fairly straightforward, but some agencies, due to unfamiliarity with the technology, request information that can delay installing the equipment. The agencies sometimes require construction "over-design," which can increase installation costs.

As mentioned earlier, operation and interconnection standards are not yet available for small CHP units in most states. Fire departments must ensure that there are no fire and safety hazards; with the potential installation of small units in common places such as federal buildings serving the public,, such units come under much closer scrutiny. Likewise, building and construction inspectors' lack of familiarity with the units can result in requirements that exceed current standards and codes for conventional technology. Because CHP equipment may be required to install air pollution control technology, hazardous materials (e.g., ammonia, sulfuric acid) may be 
involved. Additional approvals are needed to ensure onsite safety and proper handling and transport of hazardous materials, as well as to ensure that measures are taken to minimize and eliminate accidental releases of hazardous materials.

For units that may be sited in neighborhood communities, issues that arise include noise and visual/aesthetics, as well as air quality impacts for certain types of CHP units. Land use issues arise if there is a concern with zoning or proximity to sensitive receptors such as schools, hospitals, day care centers and environmentally sensitive areas. For rapidly growing areas, amendments must be made to zoning and/or the land use plans if a proposed site is not properly zoned; this can be time-consuming and involve not only an agency review but community acceptance.

\section{Summary}

Difficulties in permitting and siting CHP may add unacceptably high costs to some CHP projects. Historically, permitting requirements and fees have been developed based on very large power generation projects usually undertaken by electric utilities. Many of these costs tend to be independent of the project's size. For large projects (such as central generating plants), these costs are relatively small. However, to the extent that these permitting and siting costs are fixed, they can affect the feasibility of CHP facilities that are of a relatively small scale. Also, large projects can take advantage of economies of scale associated with technologies required to meet air emission standards. When limits on emissions or BACT requirements are determined by the technologies that can be justified for large generating units, costs to small CHP facilities may be prohibitive.

The barriers to $\mathrm{CHP}$ will be reduced as the regulatory requirements for emissions recognize the effect of CHP's source energy efficiency. Also, barriers to future development of CHP can be reduced if permit and siting requirements can be standardized to fit CHP's unique characteristics and smaller scale. 


\section{DISTRIBUTION}

1. S. W. Hadley, 3147, MS-6070

2. K. L. Kline, 3147, MS-6070

3. S. E. Livengood, 3147, MS-6070

4. P. D. Fairchild, 3147 , MS-6070

5. S. Fischer, 3147 , MS-6070

6. P. J. Hughes, 3147, MS-6070

7. W. C. Craddick, 3147, MS-6070

8. J. E. Christian, 3147, MS-6070

9. E. C. Fox, $4500 \mathrm{~N}$, MS-6189

10. M. A. Brown, 4500N, MS-6186

11. Central Research Library, 4500N, MS-6191

12. Document Reference Section, 4500N, MS-6191

13. Laboratory Records, 4500N, MS-6285

14-21. S. S. Johnsen, 3147, MS-6070

22. J. Van Dyke, 3156, MS 6073

23. J. Kelley, 3147 , MS 6070

24-31. L. Stansberry, 3147, MS 6070 (for ORNL CHP core team)

32. R. B. Shelton, $4500 \mathrm{~N}$, MS 6186

33. ORNL Central Research Library

34. Laboratory Records

35. S. Herrera, U.S. DOE HQ, Forrestal, 1000 Independence Avenue S.W., Wash. D.C. 20585

36. B. Shearer, U.S. DOE HQ, Forrestal, 1000 Independence Avenue S.W., Wash. D.C. 20585

37. T. Strajnic, U.S. DOE HQ, Forrestal, 1000 Independence Avenue S.W., Wash. D.C. 20585

38. R. Combes, U.S. DOE Atlanta Regional Office, 75 Spring Street, Suite 200, Atlanta, GA 303083308

39. A. Jhaveri, U.S DOE Seattle Regional Office, 800 Fifth Avenue, Suite 3950, Seattle, WA 981043122

40. C. Tremper, McNeil Technologies, 6564 Loisdale Court, Suite 600, Springfield, VA 22150

41. T. Rooney, McNeil Technologies, 6564 Loisdale Court, Suite 600, Springfield, VA 22150 